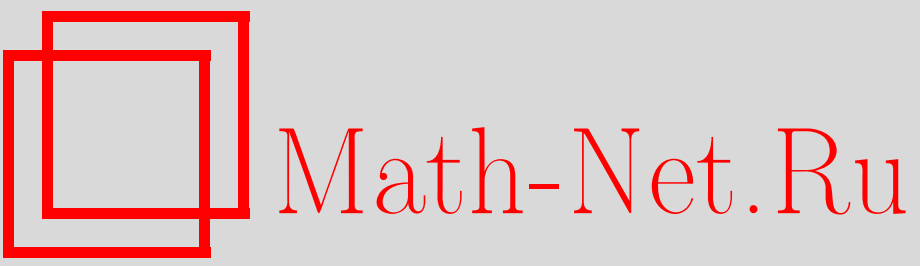

Л. А. Бекларян, Группы гомеоморфизмов прямой и окружности. Топологические характеристики и метрические инварианты, УМН, 2004, том 59, выпуск 4, 3-68

DOI: https://doi.org/10.4213/rm758

Использование Общероссийского математического портала Math-Net.Ru подразумевает, что вы прочитали и согласны с пользовательским соглашением

http: //www . mathnet.ru/rus/agreement

Параметры загрузки:

IP : 3.91 .87 .62

26 апреля 2023 г., 15:45:32 


\section{ГРУППЫ ГОМЕОМОРФИЗМОВ ПРЯМОЙ И ОКРУЖНОСТИ. ТОПОЛОГИЧЕСКИЕ ХАРАКТЕРИСТИКИ И МЕТРИЧЕСКИЕ ИНВАРИАНТЫ}

\section{Л.А. БЕКЛАРЯН}

Обзор посвящен исследованиям по топологическим, алгебраическим и комбинаторньм характеристикам, а также метрическим инвариантам для произвольных групп гомеоморфизмов прямой и окружности. Установлены взаимосвязи между этими характеристиками, изучены важнейшие метрические инварианты в виде инвариантных, проективно-инвариантных и $\omega$-проективно-инвариантных мер, описаны основные "препятствия" для существования таких метрических инвариантов.

Библиография: 53 названия.

\section{СОДЕРЖАНИЕ}

Введение

$\S 1$. Основные определения

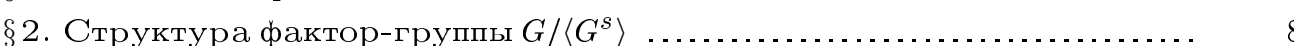

$\S 3$. Структура минимальных множеств и критерии их существования $\ldots . .11$

$\S 4$. Уточнение теоремы о фактор-группе $G /\left\langle G^{s}\right\rangle$ с использованием свойств

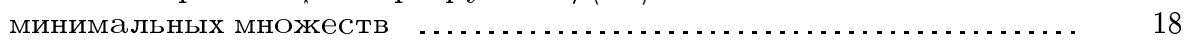

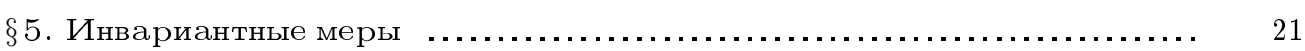

5.1 Топологические и алгебраические критерии существования инвариантной

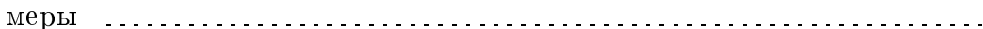

5.2. Эргодические свойства инвариантной меры ......................

5.3. Число сдвига и число вращения. Характеры, псевдохарактеры, квазиха-

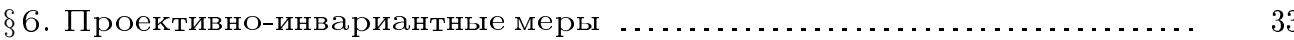

6.1. Характеры и канонические подгруппы, определяемые числом растяже-

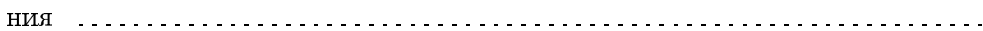

6.2. Топологические и алгебраические критерии существования проектив-

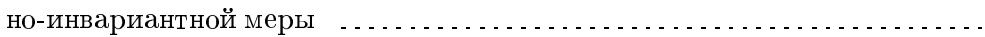

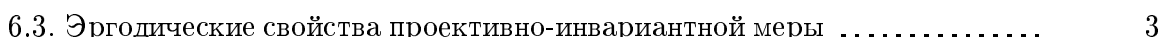

$\S 7 . \omega$-проективно-инвариантные меры

7.1. Топологический признак существования 0 -максимальных и 1 -максимальных подгрупп. Структура таких подгрупп

7.2. Топологические признаки существования $\omega$-проективно-инвариантной меры 
$\S 8$. Метрические инварианты и полусопряженность ....................

§9. О комбинаторных свойствах групп гомеоморфизмов прямой и окруж-

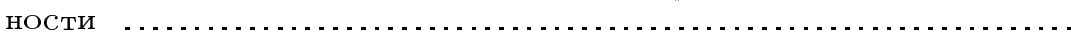

9.1. Проблема существования инвариантных и проективно-инвариантных мер и связанные с ними комбинаторные "препятствия"

9.2. Об аналогах альтернативы Титса для групп гомеоморфизмов прямой и

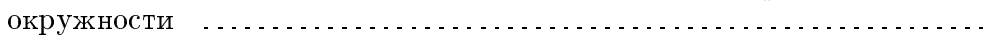

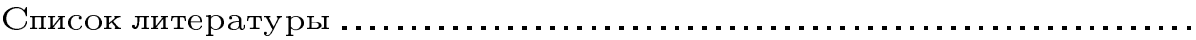

\section{Введение}

Различные вопросы алгебры, анализа, теории динамических систем приводят к необходимости исследования групп гомеоморфизмов локально-компактного пространства [1]-[41]. Первые работы в этом направлении связаны с исследованиями А. Пуанкаре поведения решений дифференциального уравнения на торе $\mathbb{T}^{2}$, которые породили задачу описания структуры гомеоморфизма единичной окружности на себя. Решение этой задачи связано с именами Пуанкаре, Данжуа, Боголюбова, Крылова, Колмогорова, Арнольда, Мозера и других [34], [2], [4], [5], [27], [38]. Гомеоморфизм окружности характеризуется важнейшим инвариантом, называемым числом врашения. Со значением этого инварианта (рациональным или иррациональным) связаны: существование неподвижных точек для какой-либо степени гомеоморфизма; сушествование замены переменной, приводящей данньй гомеоморфизм к вращению окружности; вопрос о гладкости замены переменной, приводяшей данный диффеоморфизм к врашению окружности. Всякому гомеоморфизму окружности соответствует эквивалентньй ему объект - коммутативная группа гомеоморфизмов прямой, сохраняющих ориентацию, с двумя образуюшими. Одна из образуюших является накрытием исходного гомеоморфизма, а вторая образующая - преобразование сдвига на единицу, т.е. $q(t)=t+1$.

В теории функций комплексных переменных исследование групы квазиконформных преобразований верхней полуплоскости, изложенное в книге Л.В. Альфорса [1], сводится к изучению квазисимметрической групшы гомеоморфизмов $\mathbb{R}$, порожденной группой граничных следов исходной групшы. В частности, вопрос о квазисимметрической сопряженности групш квазисимметрических преобразований групе афффинных преобразований прямой исследовался в работах [24], [14].

В геометрии исследование компоненты Новикова слоения коразмерности один сводится к изучению представления фундаментальной групшы этой компоненты в групу гомеоморфизмов прямой [39]-[41], [32], [33], [25]. В частности, класс слоений, приспособленных для этой операции, - это слоения коразмерности один, порожденные потоками Аносова.

Другой класс задач связан с исследованием дифференщиального уравнения с отклоняюшимся аргументом

$$
\dot{x}(t)=f\left(t, x\left(g_{1}(t)\right), \ldots, x\left(g_{s}(t)\right)\right), \quad t \in I,
$$

Работа выполнена при поддержке гранта Президента РФ для поддержки ведущих научных школ (грант № 00-15-96107) и Российского фонда фундаментальных исследований (грант № 0301-00174). 
где $I$ - отрезок, полупрямая или прямая $\mathbb{R}$, а функции отклонения $g_{j}(t), j=\overline{1, s}$, задают гомеоморфизмы $g_{j}: \mathbb{R} \rightarrow \mathbb{R}, j=\overline{1, s}$, сохраняющие ориентацию, и, соответственно, образуют конечно-порожденную группу гомеоморфизмов $G=\left\langle g_{j}, j=\overline{1, s}\right\rangle$. Оказывается, что важнейшие свойства вариационных задач для систем с отклоняюшимся аргументом связаны со структурой групшы гомеоморфизмов $G[6]-[8]$. В частности, со структурой группы гомеоморфизмов $G$ связана проблема классификации дифференщиальных уравнений с отклоняющимся аргументом [15].

В алгебре одной из самостоятельных задач является исследование правоупорядочиваемых групп [42]. Известно, что правоупорядочиваемая группа может быть реализована как группа автоморфизмов упорядоченного множества. Правоупорядоченная счетная группа может быть реализована как группа гомеоморфизмов прямой, сохраняюших ориентацию [19]. Независимо от работы [19], на этот результат автору указал Р. И. Григорчук. Более того, им было отмечено, что такие реализации упорядочиваемых групп в виде групп гомеоморфизмов прямой обладают дополнительньм важным свойством: графики различных элементов образуют кортеж, т.е. график одного из них расположен над графиком другого, хотя касание допустимо.

Отмеченные вьше задачи призваны показать, что такие специальные групшы гомеоморфизмов локально компактного пространства, как групш гомеоморфизмов прямой, являются универсальными объектами теории груп.

Данньй обзор посвящен исследованию важнейших характеристик групп гомеоморфизмов прямой и окружности. К ним относятся топологические, метрические, алгебраические и комбинаторные характеристики, и большая часть обзора посвящена изучению взаимосвязей между характеристиками различной природы.

Из топологических характеристик следует выделить минимальные множества, орбиты точек, множество неподвижных точек элементов групшы. Оказьвается, что каждое из этих множеств имеет каноническую структуру, которая будет в дальнейшем описана. Будут сформулированы различные критерии и признаки наличия тех или иных свойств для топологических характеристик. В частности, конечно-порожденные группы отличаются наличием непустого минимального множества.

Метрический инвариант в виде инвариантной меры является одним из центральных объектов теории динамических систем. К таким задачам относится, в частности, проблема сушествования инвариантной меры для различных групп гомеоморфизмов локально компактного пространства и описания ее свойств. По истории этого вопроса и важнейшим результатам существует исчерпьваюший обзор Д. В. Аносова [2] с полным списком литературы. Важнейшим вопросом при исследовании групп гомеоморфизмов прямой и их классификации является описание метрических инвариантов как в виде инвариантных и проективно-инвариантных мер, так и в виде более общих $\omega$-проективно-инвариантных мер, где $\omega$ - кардинальное число [11]. Будут сформулированы различные критерии и признаки существования метрических инвариантов, а также изучены их носители и эргодические свойства. В частности, для групп гомеоморфизмов прямой необходимым условием наличия метрического инварианта является непустота минимального множества.

С каждой группой гомеоморфизмов прямой связан ряд канонических подгрупш. Свойства таких канонических подгруп связаны как с метрическими и комбинаторными характеристиками, так и с конструкциями нетривиальных характеров для исходной групшы. На этом пути, в частности, будут описаны все максимальные 
нормальные подгрупшы с инвариантной мерой.

В данной работе к наиболее важным рассматриваемым комбинаторньм характеристикам относятся: субэкспоненциальность; аменабельность; отсутствие свободной подгрупшы или свободной подполугрупшы с двумя образующими у фактор-группы исходной группы по канонически выделенньм подгруппам. Изучаются связи между отмеченньми комбинаторными характеристиками и метрическими инвариантами. В частности, показано, что для групп с непустым минимальным множеством отсутствие соответствуюших комбинаторных характеристик является препятствием для сушествования инвариантной либо проективно-инвариантной меры.

В основе всех замечательных свойств групп гомеоморфизмов прямой лежит некоторое естественное отношение частичного порядка для групп гомеоморфизмов прямой, сохраняющих ориентацию [9], и фундаментальная теорема Гёльдера об архимедовых группах [43]. Вместе с тем, к примеру, известные до сих пор доказательства сушествования инвариантной меры для груп гомеоморфизмов прямой, сохраняющих ориентацию, использовали те или иные комбинаторные свойства груп (субэкспоненциальность, абелевость, аменабельность, конечная порожденность) либо наличие некоторых дифференциальных свойств (рассмотрение специальных классов диффеоморфизмов) [20], [32], [33], [35]-[37]. В этой связи природа существования инвариантной меры (и других метрических инвариантов) не совсем была ясна. Подход, принятьй в работах [9]-[13], [16] для изучения групп гомеоморфизмов $\mathbb{R}$, сохраняющих ориентацию, позволяет полностью исследовать природу существования не только инвариантной меры (априори не предполагая никаких ограничений), но и более общего метрического инварианта в виде $\omega$-проективно-инвариантной меры. Указанный подход положен в основу данного обзора. Важно отметить, что доказательства всех основных анонсируемых результатов построены на использовании внутренних конструкций для гомеоморфизмов прямой, сохраняющих ориентацию, и многократном использовании фундаментальной теоремы Гёльдера об архимедовых группах. В этой связи автор хотел бы выразить свою признательность Д. В. Аносову, которьй при подготовке работы [6] обратил мое внимание на важность теоремы Гёльдера для рассматриваемой там задачи.

За рамками данной статьи остались такие важные вопросы, как исследование специальных групп $G$ гомеоморфизмов прямой и окружности, в частности: групп диффеоморфизмов прямой, сохраняющих ориентацию (возможность уточнения структуры минимальных множеств в виде теорем Данжуа, сушествование инвариантной меры и другие вопросы); групп кусочно-линейных гомеоморфизмов; группы $P S L(2, \mathbb{R})$. Мы также не будем касаться груп ограниченных когомологий $H_{B}^{k}(G: \mathbb{R}), H_{B}^{k}(G: \mathbb{Z})$ и связанных с ними инвариантов. Такие исследования проведены в работах $[22],[21]$, [32], [33], [25], [35]-[37], [29], [30], [18], [19], [44]-[51], [42]. По многим из отмеченных вопросов существует исчерпывающий обзор Э. Гиза [19] с полным списком литературы.

\section{$\S$ 1. Основные определения}

В дальнейшем $X$ обозначает либо $\mathbb{R}$, либо $S^{1}$. Пусть:

$\operatorname{Homeo}(X)$ - группа всех гомеоморфизмов $X$, а Ноmeо+ $(X)$ - нормальная подгруппа сохраняюших ориентацию гомеоморфизмов;

$\widehat{\operatorname{Homeo}}\left(S^{1}\right)$ - группа всех гомеоморфизмов прямой, являюшихся накрытиями 
гомеоморфизмов из Ноmео $\left(S^{1}\right)$, а $\widetilde{\operatorname{Homeo}}_{+}\left(S^{1}\right)$ - нормальная подгруппа сохраняющих ориентацию гомеоморфизмов.

Наряду с групшой $G \subseteq \operatorname{Homeo}(X)$ будем рассматривать ее нормальную подгрупу

$$
G_{+}=G \cap \mathrm{Homeo}_{+}(X),
$$

принадлежащее ей объединение стабилизаторов точек $X$

$$
G^{s}=\left\{g: g \in G_{+} ; \exists t \in X, g(t)=t\right\}
$$

и подмножества

$$
\begin{gathered}
G_{\infty}^{s}=\left\{g: g \in G^{s}, \inf \{t: g(t)=t\}=-\infty, \sup \{t: g(t)=t\}=+\infty\right\}, \\
C_{G}=\left(G \backslash G^{s}\right) \cup G_{\infty}^{s}
\end{gathered}
$$

в случае $X=\mathbb{R}$, которые не всегда являются подгруппами. Заметим, что нормальная подгруппа $G_{+}$является подгруппой индекса не более двух. Для произвольного множества гомеоморфизмов $Q$ через $\langle Q\rangle$ будем обозначать группу, порожденную этими гомеоморфизмами, а также определим топологическую характеристику в виде множества неподвижных точек

$$
\operatorname{Fix} Q=\{t: t \in X, \forall q \in Q, q(t)=t\} .
$$

Гомеоморфизм $g$ назьвается свободно действуюшим, если Fix $\langle g\rangle=\varnothing$; сдвигом, если он задается функцией $t+b$; аффинным преобразованием, если он задается функцией $a t+b$.

ЗАМЕЧАнИЕ 1.1. Для любого $g \in G$ вьполнены условия: $g^{-1} G^{s} g=G^{s}$, $g^{-1} G_{\infty}^{s} g=G_{\infty}^{s}, g^{-1} C_{G} g=C_{G}$. Поэтому группы $\left\langle G^{s}\right\rangle,\left\langle G_{\infty}^{s}\right\rangle$ и $\langle C\rangle$ являются нормальными подгруппами исходной группы $G$.

Пусть $\bar{g}(t)=t+1$ - сдвиг на единицу, а $\lambda: \mathbb{R} \rightarrow S^{1}$ отображение вида $\lambda(t)=t-[t]$, где [· ] означает целую часть числа. Очевидно, что $\bar{g} \subseteq \widehat{\mathrm{Homeо}_{+}}\left(S^{1}\right)$ и коммутирует со всеми элементами групшы $\overparen{\operatorname{Homeo}}\left(S^{1}\right)$. Отображение $\lambda$ индуцирует естественньй эпиморфизм $\pi: \widetilde{\operatorname{Homeo}}\left(S^{1}\right) \rightarrow \operatorname{Homeo}\left(S^{1}\right)$, для которого $\operatorname{ker} \pi=\langle\bar{g}\rangle$. Для любой групшы $G \subseteq \operatorname{Homeo}\left(S^{1}\right)$ через $\widehat{G}$ будем обозначать подгруппу групшы $\widehat{\operatorname{Homeo}}\left(S^{1}\right)$ вида $\widehat{G}=\left\langle\left\{l_{g}\right\}_{g \in G}, \bar{g}\right\rangle$, где $l_{g}$ для любого $g \in G$ обозначает гомеоморфизм прямой, являюшийся накрытием гомеоморфизма $g$ и нормированный условием $0 \leqslant l_{g}(0)<1$. Через $\pi_{\widehat{G}}$ будем обозначать ограничение эпиморфизма $\pi$ на подгруппу $\widehat{G}$. Справедливо следующее простое предложение.

ПРЕДЛОЖЕНИЕ 1.1. Пусть $G \subseteq \operatorname{Homeo}\left(S^{1}\right) . \quad$ Тогда $\operatorname{Im} \pi_{\widehat{G}}=G, \operatorname{ker} \pi_{\widehat{G}}=\langle\bar{g}\rangle$, $\pi_{\widehat{G}}\left(\widehat{G}^{s}\right)=G^{s}$.

Доказательство непосредственно следует из определения.

Если $A \subseteq X$ - некоторое подмножество, то $P(A)$ будет обозначать множество его предельных точек. Если $A$ - пустое подмножество, то по определению положим $P(A)=\varnothing$.

Для групшы $G \subseteq$ Homeo+ $_{+}(X)$ через $p_{G}$ будем обозначать стандартньй гомоморфизм группы $G$ в фактор-группу $G /\left\langle G^{s}\right\rangle$. 


\section{$\S$ 2. Структура фактор-группы $G /\left\langle G^{s}\right\rangle$}

Хорошо известно, что группа $G \subseteq$ Нотео+ $_{+}(X)$ свободно действуюших гомеоморфизмов изоморфна некоторой подгруппе аддитивной группы $X$ [31]. Для произвольной группы $G \subseteq \mathrm{Homeo}_{+}(X)$ ситуация усложняется в связи с появлением нетривиальных элементов группы, имеющих неподвижные точки (нетривиальные стабилизаторы точек на $X$ ). Такие элементы групш $G$ образуют множество $G^{s}$. При этом множество $G^{s}$ не обязано быть подгруппой. Минимальной подгруппой, связанной с множеством всех стабилизаторов точек на $X$, является групша $\left\langle G^{s}\right\rangle$, порожденная множеством $G^{s}$. Подгруппа $\left\langle G^{s}\right\rangle$ является нормальной, а в случае группы $G$ со свободно действуюшими гомеоморфизмами подгрупша $\left\langle G^{s}\right\rangle$ тривиальная, т.е. $\left\langle G^{s}\right\rangle=\langle e\rangle$. В связи с изложенньм естественно надеяться, что для произвольной групшы $G \subseteq \mathrm{Homeo}_{+}(X)$ фактор-группа $G /\left\langle G^{s}\right\rangle$ также будет иметь простую структуру. Это оказывается действительно так, чему и посвящен основной результат данного параграфа - теорема о фактор-группе (теорема 2.1). В силу этого при исследовании произвольных групп $G \subseteq$ Homeo $_{+}(X)$ определяюшей оказьвается структура фактор-групшы $G /\left\langle G^{s}\right\rangle$.
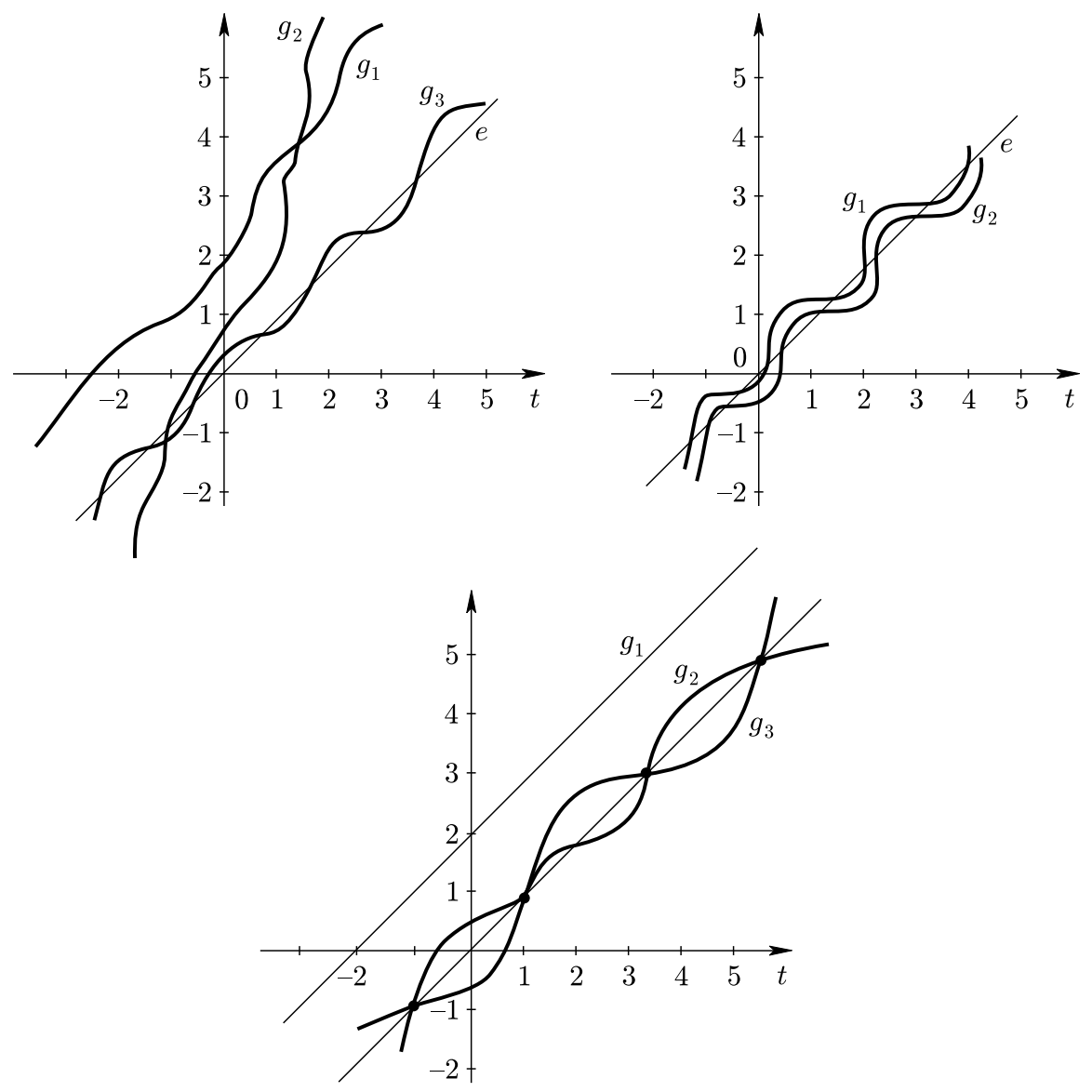

Для трех примеров конечно-порожденных групп на рисунках приведены графики их образующих. График диагонали задает единичньй элемент группы. 
В первом примере для группы $G=\left\langle g_{1}, g_{2}, g_{3}\right\rangle$ элементы $g_{2}, g_{3}$ принадлежат множеству $G^{s}$. Более того, элемент $g_{3}$ принадлежит также и подмножеству $G_{\infty}^{s}$ множества $G^{s}$, а элемент $g_{2}$ принадлежит подмножеству $G^{s} \backslash G_{\infty}^{s}$. Элемент $g_{1}$ принадлежит множеству $G \backslash G^{s}$. В таком случае множество $G^{s}$ не является подгруппой, так как элементы $g_{2}$ и $g_{2}^{-1} g_{1}$ принадлежат множеству $G^{s}$, а их произведение $g_{2} g_{2}^{-1} g_{1}=g_{1}$ не принадлежит $G^{s}$. Заметим, что для такой группы множество $\mathrm{Fix} G^{s}$ пустое.

Во втором примере для группы $G=\left\langle g_{1}, g_{2}\right\rangle$ элементы $g_{1}, g_{2}$ принадлежат множеству $G^{s}$ (более того, подмножеству $G_{\infty}^{s}$ ) и удовлетворяют условиям: $g_{1}(t+1)=g_{1}(t)+1$, $g_{2}(t)=g_{1}(t)-\alpha, \alpha>0$. Очевидно, что элемент $g_{1} g_{2}$ не принадлежит множеству $G^{s}$, множество $G^{s}$ не образует подгрупу, совпадает с $G_{\infty}^{s}$, а множество Fix $G^{s}$ также является пустьм.

В третьем примере для групшы $G=\left\langle g_{1}, g_{2}, g_{3}\right\rangle$ элементы $g_{2}, g_{3}$ принадлежат множеству $G_{\infty}^{s}$, имеют обшие неподвижные точки $\{(2 k-1): k \in \mathbb{Z}\}$. Множество $G^{s}$ образует подгруппу, совпадает с $G_{\infty}^{s}$, а множество Fix $G^{s}$ непусто и совпадает с множеством $\{(2 k-1): k \in \mathbb{Z}\}$.

Существуют примеры групп $G \subseteq \mathrm{Homeо}_{+}(\mathbb{R})$ (не конечно порожденных), для которых $G=G_{\infty}^{s}$, а множество Fix $G^{s}$ пустое.

Для произвольной группы $G \subseteq$ Нотео+ $_{+}(\mathbb{R})$ описание фактор-групшы $G /\left\langle G^{s}\right\rangle$ основано на сушествовании некоторого естественного отношения частичного порядка в групе $G$, которое определяется следуюшим образом: $g_{1} \preceq g_{2}$, если для всех $t \in \mathbb{R}$ одновременно $g_{1}(t) \leqslant g_{2}(t)$. Учитьвая важность теоремы о фактор-групше, мы приведем доказательство основных результатов, связанных с ней.

Вначале (лемма 2.1) изучаются свойства частичного порядка в группе $G \subseteq$ $\mathrm{HomeO}_{+}(\mathbb{R})$, определенного вьше. Затем (основная лемма) описьвается структура нормальной подгруппы $\left\langle G^{s}\right\rangle$. Оказывается, что нормальная подгрупа $\left\langle G^{s}\right\rangle$ удовлетворяет экстремальному свойству: она совпадает или с порождающим множеством $G^{s}$, или с исходной группой $G$. При некоторых дополнительных условиях структура нормальной подгрупшы $\left\langle G^{s}\right\rangle$ допускает уточнение (лемма 2.3). И наконец, на основе леммы 2.1 доказывается теорема о фактор-группе.

Лемма 2.1 [9]. Пусть задана группа $G \subseteq$ Homeо $_{+}(\mathbb{R})$, в которой определено отношение частичного порядка: $g_{1} \preceq g_{2}$, если для всех $t \in \mathbb{R}$ одновременно $g_{1}(t) \leqslant g_{2}(t)$. Если $g_{1}\left\langle G^{s}\right\rangle \neq g_{2}\left\langle G^{s}\right\rangle$, то между любыми әлементами левых смежных классов $g_{1}\left\langle G^{s}\right\rangle, g_{2}\left\langle G^{s}\right\rangle$ виполняется одно и то же отношение порядка.

ДокаЗАтЕльСтво. Если $g_{1}\left\langle G^{s}\right\rangle \neq g_{2}\left\langle G^{s}\right\rangle$, то очевидно, что $g_{1}^{-1} g_{2} \notin\left\langle G^{s}\right\rangle$ и, в частности, $g_{1}^{-1} g_{2} \notin G^{s}$. В таком случае между элементами $g_{1}, g_{2}$ сушествует отношение строгого порядка, т.е. для всех $t \in \mathbb{R}$ одновременно либо $g_{1}(t)>g_{2}(t)$, либо $g_{1}(t)<g_{2}(t)$. Заметим, что для любых $g_{1}, g_{2} \in G, \bar{g} \in\left\langle G^{s}\right\rangle, g_{1}\left\langle G^{s}\right\rangle \neq g_{2}\left\langle G^{s}\right\rangle$, соотношения $g_{2} \succ g_{1}, g_{2} \bar{g} \succ g_{1}, g_{2} \succ g_{1} \bar{g}$ эквивалентны. Поэтому отношение порядка между двумя произвольными элементами левых смежных классов $g_{1}\left\langle G^{s}\right\rangle$ и $g_{2}\left\langle G^{s}\right\rangle$ одно и то же.

На основе леммы 2.1, пользуясь свойствами гомеоморфизмов прямой, сохраняюших ориентацию, доказана следуюшая основная лемма. 
Основная ЛЕмма 2.2 [9]. Пусть задана групna $G \subseteq$ Homeo+ $_{+}(X)$. Тогда сnраведливо хотя би одно из условий: $G^{s}=\left\langle G^{s}\right\rangle$ или $G=\left\langle G^{s}\right\rangle$.

ДокАЗАТЕльство. В случае $X=\mathbb{R}$ доказательство построено на использовании отнощения частичного порядка, определенного в лемме 2.1 (см. [9]).

Пусть $G^{s} \subset\left\langle G^{s}\right\rangle \subset G$. Из условия $G^{s} \neq\left\langle G^{s}\right\rangle$ следует сушествование элементов $g_{1}, g_{2} \in G^{s}, \bar{g} \in\left\langle G^{s}\right\rangle \backslash G^{s}$ таких, что $\bar{g}=g_{1} g_{2}$. Не нарушая обшности, можем полагать, что $\bar{g} \succ e$.

Из условия $\left\langle G^{s}\right\rangle \neq G$ следует сушествование элемента $g \in G \backslash\left\langle G^{s}\right\rangle$. Так как элементы $g, \bar{g}$ принадлежат разным смежным классам $\left(\bar{g} \in e\left\langle G^{s}\right\rangle\right)$, то между ними сушествует отношение порядка. Не нарушая общности, можем полагать, что для всех $t \in \mathbb{R}$ одновременно $g(t)>\bar{g}(t)$. В противном случае это условие вьполняется для некоторой степени $g^{m}, m \in \mathbb{Z}$, элемента $g$, которую мы и выберем вместо $g$. Тогда найдется натуральное $n$ такое, что

$$
\bar{g}^{n} \prec g \prec \bar{g}^{n+1} \text {. }
$$

Элементы $\bar{g}^{n}, \bar{g}^{n+1}$ принадлежат одному и тому же левому смежному классу $e\left\langle G^{s}\right\rangle$, а по предыдушей лемме отношение порядка между двумя произвольными элементами двух левых смежных классов одно и то же. Полученное противоречие и доказьвает лемму для случая $X=\mathbb{R}$.

Проведем доказательство для случая $X=S^{1}$. Наряду с групой $G \subseteq \operatorname{Homeo}_{+}\left(S^{1}\right)$ рассмотрим соответствуюшую группу $\widehat{G} \subseteq \widehat{\mathrm{Homeo}_{+}}\left(S^{1}\right)$ и эпиморфизм $\pi_{\widehat{G}}: \widehat{G} \rightarrow G$ из предложения 1.1. Из предложения 1.1 следует, что $\pi_{\widehat{G}}(\mathscr{T})=\left\langle G^{s}\right\rangle$, где $\mathscr{T}=\left\langle\widehat{G}^{s}, \bar{g}\right\rangle$. В таком случае существуют эпиморфизм $\widehat{G} /\left\langle\widehat{G}^{s}\right\rangle \rightarrow \widehat{G} / \mathscr{T}$ и изоморфизм $\widehat{G} / \mathscr{T} \simeq G /\left\langle G^{s}\right\rangle$. Из нетривиальности фактор-групшы $G /\left\langle G^{s}\right\rangle$ и, соответственно, фактор-групшы $\widehat{G} / \mathscr{T}$ будет следовать нетривиальность фактор-групшы $\widehat{G} /\left\langle\widehat{G}^{s}\right\rangle$. Тогда по предыдущему пункту должно вьполняться условие $\left\langle\widehat{G}^{s}\right\rangle=\widehat{G}^{s}$. Так как $\pi_{\widehat{G}}\left(\widehat{G}^{s}\right)=G^{s}$, то отсюда и будет следовать условие $\left\langle G^{s}\right\rangle=G^{s}$.

В действительности о подгрупе $\left\langle G^{s}\right\rangle$ мы можем получить еще некоторую дополнительную информацию.

ЛЕмма $2.3[9]$. Пусть задана группа $G \subseteq \mathrm{Homeo}_{+}(\mathbb{R})$, для которой $G \neq\left\langle G^{s}\right\rangle$. Тогда $\left\langle G^{s}\right\rangle=G^{s}=G_{\infty}^{s}$.

ДокАЗАТЕЛЬСтво. Очевидно, что $G_{\infty}^{s} \subseteq G^{s}$. Покажем, что $G^{s}=G_{\infty}^{s}$. Доказательство проведем от противного. Предположим, что $G^{s} \backslash G_{\infty}^{s} \neq \varnothing$. Выберем $g_{1} \in G \backslash\left\langle G^{s}\right\rangle$ и $g_{2} \in G^{s} \backslash G_{\infty}^{s}$. Тогда найдутся $n \in \mathbb{Z}$ и $t \in \mathbb{R}$, для которых выполняется условие $g_{1}(t)=g_{2}^{n}(t)$, что противоречит лемме 2.1. Остается показать, что $\left\langle G^{s}\right\rangle=G^{s}$. Предположим, что $\left\langle G^{s}\right\rangle \neq G^{s}$. Тогда найдется элемент $g_{3}$ со свойствами $g_{3} \in\left\langle G^{s}\right\rangle, \quad g_{3} \in G \backslash G^{s}$. Так как сушествует элемент $g_{1} \in G \backslash\left\langle G^{s}\right\rangle$, то наличие элемента $g_{3}$ также противоречит лемме 2.1 .

ЗАмечание 2.1. Пусть задана группа $G \subseteq \widetilde{\text { Homeo+ }_{+}}\left(S^{1}\right)$. Тогда $G^{s}=G_{\infty}^{s}$.

ДокаЗАТЕльство. Заметим, что для любого $g \in G$ множество $\operatorname{Fix}\langle g\rangle$ инвариантно относительно сдвига $\bar{g}(t)=t+1$, откуда и следует условие $G^{s}=G_{\infty}^{s}$. 
Теперь мы можем сформулировать теорему о фактор-группе, которая является обобшением теоремы Гёльдера [31] о коммутативности групшы $G \subseteq \mathrm{Homeo}_{+}(\mathbb{R})$ свободно действуюших гомеоморфизмов на прямой (для любого $g \in G, g \neq e$, выполняется условие $\operatorname{Fix}\langle g\rangle=\varnothing)$.

Tеорема о ФАКТОР-ГРУППе 2.1 [9]. Пусть $G \subseteq$ Homeo+ $_{+}(X)$. Тогда факторгруппа $G /\left\langle G^{s}\right\rangle$ коммутативна и изоморфна некоторой подгруппе аддитивной zpynnbl $X$.

ДокАЗАТЕльство. В случае $X=\mathbb{R}$ доказательство теоремы построено на использовании основной леммы и теоремы Гёльдера об архимедовых группах.

Покажем, что фактор-группа $G /\left\langle G^{s}\right\rangle$ является линейно упорядоченной и архимедовой. В группе $G$ сушествует отношение частичного порядка, указанное в лемме 2.1. По этой же лемме отношение порядка между двумя произвольньми элементами левых смежных классов $g_{1}\left\langle G^{s}\right\rangle$ и $g_{2}\left\langle G^{s}\right\rangle$ одно и то же. Значит, можно корректно определить отношение частичного порядка между любыми левыми смежными классами следуюшим образом: если $g_{1}\left\langle G^{s}\right\rangle, g_{2}\left\langle G^{s}\right\rangle$ - левые смежные классы группы $G$ по подгрупе $\left\langle G^{s}\right\rangle$, то $g_{1}\left\langle G^{s}\right\rangle \preceq g_{2}\left\langle G^{s}\right\rangle$ тогда и только тогда, когда $g_{1} \preceq g_{2}$.

Пусть заданы произвольные левые смежные классы $g_{1}\left\langle G^{s}\right\rangle$ и $g_{2}\left\langle G^{s}\right\rangle$ такие, что $g_{1} \succ e, g_{2} \succ e$. Для любых таких элементов $g_{1}$ и $g_{2}$ найдется натуральное $n$ такое, что $g_{2}^{n} \succ g_{1}$. Следовательно, $g_{2}^{n}\left\langle G^{s}\right\rangle \succ g_{1}\left\langle G^{s}\right\rangle$, откуда и следует архимедовость фактор-групшы $G /\left\langle G^{s}\right\rangle$. Тогда по теореме Гёльдера [43] фактор-группа $G /\left\langle G^{s}\right\rangle$ коммутативна и изоморфна подгруппе аддитивной группы действительных чисел.

Проведем доказательство для случая $X=S^{1}$. Наряду с групой $G \subseteq \operatorname{Homeo}_{+}\left(S^{1}\right)$ рассмотрим соответствуюшую группу $\widehat{G} \subseteq \widehat{\text { Нотео }_{+}}\left(S^{1}\right)$ и эпиморфизм $\pi_{\widehat{G}}: \widehat{G} \rightarrow G$ из предложения 1.1. Из предложения 1.1 следует, что $\pi_{\widehat{G}}(\mathscr{T})=\left\langle G^{s}\right\rangle$, где $\mathscr{T}=\left\langle\widehat{G}^{s}, \bar{g}\right\rangle$. В таком случае сушествуют изоморфизм $\widehat{G} / \mathscr{T} \simeq G /\left\langle G^{s}\right\rangle$ и эпиморфизм $\widehat{G} /\left\langle\widehat{G}^{s}\right\rangle \rightarrow$ $\widehat{G} / \mathscr{T}$. Ядром эпиморфизма является циклическая подгрупша с образуюшей $\bar{g}\left\langle G^{s}\right\rangle$ (левый смежньй класс элемента $\bar{g}$ по подгруппе $\left.\left\langle G^{s}\right\rangle\right)$. Так как фактор-группа $\widehat{G} /\left\langle\widehat{G}^{s}\right\rangle$ изоморфна подгрупе аддитивной групшы $\mathbb{R}$, то фактор-групп $G / G^{s}$ будет изоморфна подгруппе групшы $S^{1}$.

Теорема о фактор-группе является центральной при исследовании метрических инвариантов. В частности, в $\S 5$ будет показано, что из нетривиальности фактор-группы $G /\left\langle G^{s}\right\rangle$ следует сушествование $G$-инвариантной меры.

Для специальных групп гомеоморфизмов прямой, сохраняющих ориентацию, утверждение теоремы о фактор-группе было получено в работах [31], [39], [35]-[37]. В работе [31] исследовались свободно действуюшие групшы, в [39] - конечно-порожденные групшы, не содержащие свободных подполугрупп с двумя образуюшими, а в [35]-[37] - счетные групшы $G$ с общей неподвижной точкой для элементов, имеющих хотя бы одну неподвижную точку, т.е. счетные группы $G$ со свойством Fix $G^{s} \neq \varnothing$.

\section{§ 3. Структура минимальных множеств и критерии их существования}

Среди топологических характеристик групп гомеоморфизмов прямой, сохраняюших ориентацию, выделяются минимальные множества. Важность минимальных множеств определяется тем, что в случае их “нетривиальности” орбиты точек обладают 
некоторыми каноническими свойствами, а сами минимальные множества определяют важнейшие характеристики метрических инвариантов. К ранним исследованиям по минимальным множествам относятся работы Данжуа для циклической группы гомеоморфизмов окружности [27]. Для произвольных груп гомеоморфизмов окружности $G \subseteq$ Homeо+ $_{+}\left(S^{1}\right)$ (компактньй случай) непустое минимальное множество всегда сушествует, а структура минимального множества в точности совпадает со структурой минимального множества для циклической группы. Как наиболее ранняя работа с таким результатом автору известен курс лекций Л.В. Альфорса [52]. Интересным оказалось то, что в некомпактном случае для груп гомеоморфизмов прямой $G \subseteq$ Homeo+ $(\mathbb{R})$ структура минимального множества осталась такой же и добавился случай отсутствия минимального множества. Это и является основньм результатом данного параграффа и приводится в виде теоремы 3.1. Выделен важньй признак существования минимального множества в виде условия конечной порожденности группы $G \subseteq \mathrm{Homeo+}_{+}(\mathbb{R})$ (теорема 3.2). Приводятся и иные признаки, а также критерии существования минимального множества. В общем случае описание минимальных множеств неконструктивно, так как использует аксиому выбора. Выделяется класс групп $G \subseteq$ Homeо+ $_{+}(\mathbb{R})$, важньй с точки зрения метрических инвариантов, для которых минимальные множества могут быть конструктивно описаны (теорема 3.8). При изучении ряда задач, например при исследовании следов группы квазиконформных отображений верхней полуплоскости в себя, оказьвается важной замена исходной группы гомеоморфизмов $X$ на более простую подгруппу с той же топологической сложностью (с тем же минимальным множеством). Поэтому изучение подгрупп исходной групшы и их минимальных множеств представляет большой интерес. Оказывается, что минимальное множество нормальных подгруп также удовлетворяет экстремальному свойству: или является дискретным множеством (возможно, пустьм), или совпадает с минимальным множеством исходной групшы. Результат такого типа сформулирован в лемме 3.4. Все отмеченные результаты получены для групп гомеоморфизмов $G \subseteq \mathrm{Homeo}_{+}(X)$, сохраняющих ориентацию. Для произвольной групшы $G \subseteq \operatorname{Homeo}(X)$ приводится процедура описания минимального множества по минимальному множеству максимальной нормальной подгрупшы $G_{+}$сохраняющих ориентацию гомеоморфизмов (теорема 3.9$)$.

Счетные группы $G \subseteq \mathrm{Homeo+}_{+}(\mathbb{R})$ исследовались в работах [35]-[37]. Были сформулированы признаки сушествования минимальных множеств, и при этих условиях описана структура орбит. Там же исследовались признаки сушествования минимальных множеств для специальных групп диффеоморфизмов (теорема 3.5). Минимальные множества для произвольных групп $G \subseteq$ Homeо+ $_{+}\left(S^{1}\right)$ описывались различными авторами. В частности, для счетных групп - в работах [35]-[37], для свободно действующих групп гомеоморфизмов - в работе [26]. Мы уже отмечали в предыдушем абзаце, что наиболее ранней работой по этой теме, известной автору, является курс лекций [52].

Далее будет сформулирована теорема о структуре минимального множества для груп $G \subseteq \mathrm{Homeo+}_{+}(\mathbb{R})$, а также о свойствах орбит (теорема 3.1). Следствием из него является теорема о структуре минимального множества для групा $G \subseteq$ Homeo+ $_{+}\left(S^{1}\right)$ (теорема 3.7), которая получена в работе [26]. Сформулирован критерий пустоты минимального множества (теорема 3.3 ), и в случае пустоты минимального множества уточнена структура исходной групшы $G \subseteq \mathrm{Homeo+}_{+}(\mathbb{R})$ (лемма 3.1 ). Приводит- 
ся ряд признаков существования непустого минимального множества для групп $G \subseteq$ $\left.\mathrm{Homeo+}_{+} \mathbb{R}\right)$ (предложение 3.1, теоремы 3.2, 3.4-3.6). Установлена связь между минимальными множествами групшы $G \subseteq \operatorname{Homeo+}_{+}(X)$ и ее подгрупп (леммы 3.3 и 3.4.). Для специального класса групп $G \subseteq$ Homeo+ $_{+}(X)$ конструктивно описаны минимальные множества (теорема 3.8). В дальнейшем будет показано, что такой класс групп совпадает с классом групп с инвариантной мерой. Если группа $G \subseteq \operatorname{Homeo+}_{+}(\mathbb{R})$ не принадлежит к такому специальному классу, то орбиты неограничены (лемма 3.2). Описана связь минимальных множеств групшы $G \subseteq \operatorname{Homeo}(X)$ и ее нормальной подгрупшы $G_{+}$(теорема 3.9$)$.

ОПРЕДЕЛЕНИЕ 3.1. Пусть $G \subseteq \operatorname{Homeo}(X)$. Непустое подмножество $X$ назьвается минимальным, если оно замкнуто, $G$-инвариантно и не содержит собственных замкнутых $G$-инвариантных подмножеств. Если не существует минимального множества, то по определению будем полагать, что оно пустое.

В случае единственного минимального множества его будем обозначать через $E(G)$.

Вследствие одномерности фазового пространства, минимальное множество имеет вполне определенную каноническую структуру, описание которой дается следующей теоремой.

Теорема 3.1 [10]. Пусть $G \subseteq$ Hотео+ $(\mathbb{R})$. Тогда справедливо одно из следующих взаимоисключающих утверждений:

а) любое минимальное множество дискретное и принадлежит множеству $\operatorname{Fix} G^{s}$, а множсество $\operatorname{Fix} G^{s}$ состоит из облединения минимальньх множеств;

б) минимальное множество является совершенным нигде не плотным подмножеством $\mathbb{R}$ (в этом случае оно является единственным минимальным множеством и содержится в замыкании орбиты $\overline{G(t)}$ произвольной точки $t \in \mathbb{R})$

в) минимальное множество совпадает $с \mathbb{R}$;

г) минимальное множсество пустое.

Одним из достаточных условий непустоты минимального множества является конечная порожденность рассматриваемой группы.

Теорема $3.2[11]$. Пусть $G \subseteq$ Homeо $_{+}(\mathbb{R})$ - конечно-порожденная группа. Тогда существует непустое минимальное множество.

Приведем критерий пустоты минимального множества, основанньй на топологических свойствах ее конечно-порожденных подгруп.

Теорема 3.3 [11]. Пусть $G \subseteq$ Homeo+ $_{+}(\mathbb{R})$. Для пустоты минимального множества необходимо и достаточно, чтобь следующие три условия выполнялись одновременно:

а) для любой конечно-порожденной подгруппь $\Gamma \subseteq G$ справедливо условие $\operatorname{Fix} \Gamma^{s} \neq \varnothing$;

б) для любого $n=1,2, \ldots$ существует конечно-порожденная подгруппа $\Gamma \subseteq G$, для которой $[-n, n] \cap \mathrm{Fix}^{s}=\varnothing ;$

в) для любого $n=1,2, \ldots$ существует точка $t \in \mathbb{R}$ такая, что $G(t) \cap$ $[-n, n]=\varnothing$. 
Наличие минимального множества или его отсутствие придает группе определенные свойства. Справедлива следующая важная лемма.

Лемма 3.1 [11]. Пусть $G \subseteq$ Нотео+ $(\mathbb{R})$. Если минимальное множество пусmoe, $\operatorname{mo} G=G_{\infty}^{s}$.

На основе леммы 3.1 мы можем сформулировать еше одно достаточное условие существования минимального множества, основанное на свойствах канонически выделенного подмножества $G_{\infty}^{s}$ групшы $G$.

ПРЕДЛОЖЕНИЕ 3.1. Пусть $G \subseteq$ Homeо+ $_{+}(\mathbb{R}) . \quad$ Если $G \neq G_{\infty}^{s}$, то существует непустое минимальное мнохсество.

Приведем ряд других важных достаточных условий существования минимального множества.

Tеорема $3.4[10]$. Пусть $G \subseteq$ Homeо+ $_{+}(\mathbb{R})$. Для существования непустого минимального множсества достаточно, чтобы выполнялось хотя бы одно из следующ, их условий:

a) $\operatorname{Fix} G^{s} \neq \varnothing$;

б) для любого $g \in G, g \neq e$, множество $\operatorname{Fix}\langle g\rangle$ дискретное;

в) $G$ содержит конечно-порожденную подгруппу $Q$, имеющую неограниченную орбиту.

Теорема 3.5 [33], [35]-[37]. Пусть $G \subseteq$ Hотео+ $_{+}(\mathbb{R})$ - счетная группа. Если $G$ абелева и ее әлементь принадлежсат $\operatorname{Diff}^{2}(\mathbb{R})$ или әлементь $G$ задаются аналитическими функииями, то существует непустое минимальное множество.

Существуют примеры счетных абелевых групп гомеоморфизмов прямой, сохраняющих ориентацию, для которых минимальное множество пустое.

В случае Fix $G^{s} \neq \varnothing$ по теореме 3.3 сушествует непустое минимальное множество и поведение орбит точек $t \in \mathbb{R}$ описывается теоремой 3.1. Приведем результат, уточняющий поведение орбит точек $t \in \mathbb{R}$ в случае $\mathrm{Fix} G^{s}=\varnothing$.

Лемма $3.2[10]$. Пусть $G \subseteq$ Homeo+ $_{+}(\mathbb{R}) . \quad$ Если $\operatorname{Fix} G^{s}=\varnothing$, то орбита $G(t)$ любой точки $t \in \mathbb{R}$ не ограничена ни сверху, ни снизу.

На основании теоремы 3.3 и лемм 3.1, 3.2 можно получить утверждение о сушествовании непустого минимального множества для групп $G \subseteq \widehat{\text { Homeо+ }_{+}}\left(S^{1}\right)$ $\left(G \subseteq\right.$ Homeo+ $\left._{+}\left(S^{1}\right)\right)$.

TEOPEма 3.6. Пусть $G \subseteq \widetilde{\text { Homeo }_{+}}\left(S^{1}\right)$. Тогда существует непустое минимальное множество.

ДокАЗАТЕльство. Если $G \neq G_{\infty}^{s}$, то существование непустого минимального множества следует из леммы 3.1. Пусть $G=G_{\infty}^{s}$. Если Fix $G^{s} \neq \varnothing$, то существование непустого минимального множества следует из п. а) теоремы 3.3. Более того, в этом случае непустое минимальное множество дискретное. Остается рассмотреть случай $G=G_{\infty}^{s}$, Fix $G^{s}=\varnothing$. Заметим, что для любого $g \in G$ множество $\operatorname{Fix}\langle g\rangle$ непусто и инвариантно относительно сдвига $\bar{g}(t)=t+1$. Поэтому для любого $g \in G$ 
вьполняется условие $\sup _{t \in \mathbb{R}}|g(t)-t|<1$. С другой стороны, по лемме 3.2 орбита любой точки $t \in \mathbb{R}$ не ограничена ни снизу, ни сверху. Противоречие.

Следовательно, для рассматриваемых групп случай $G=G_{\infty}^{s}$, Fix $G^{s}=\varnothing$ не peализуем, что и завершает доказательство теоремы.

СлЕдСТвИЕ 3.1. Пусть задана группа $G \subseteq \widetilde{\mathrm{HomeO}_{+}}\left(S^{1}\right) . \quad$ Если $G=G^{s}$, mo Fix $G^{s} \neq \varnothing$.

ДокАЗАТЕЛЬСТво. По замечанию 2.1 для таких групп $G^{s}=G_{\infty}^{s}$, а в последнем абзаце доказательства теоремы 3.6 показано, что случай $G=G_{\infty}^{s}$, Fix $G^{s}=\varnothing$ не реализуем.

Сформулируем аналог теоремы 3.1 о структуре минимальных множеств для групп $G \subseteq \mathrm{Homeo}_{+}\left(S^{1}\right)$.

Теорема 3.7. Пусть $G \subseteq$ Homeо $_{+}\left(S^{1}\right)$. Тогда минимальное множество непустое и справедливо одно из следующих взаимоисключающих утверждений:

а) любое минимальное множество дискретное и принадлежит множеству $\operatorname{Fix} G^{s}$, а множество $\operatorname{Fix} G^{s}$ состоит из облединения минимальных множеств;

б) минимальное множсество является совершенным нигде не плотным подмножеством $S^{1}$ (в этом случае оно является единственныц минимальным множсеством и содержится в замыкании орбиты $\overline{G(t)}$ произвольной точки $\left.t \in S^{1}\right)$;

в) минимальное множество совпадает с $S^{1}$.

ДокАЗАТЕльство. Пусть группа $\widehat{G}$ и отображения $\bar{g}, \lambda, \pi_{\widehat{G}}$ те же, что и в $\S 1$ (см. предложение 1.1.). По теореме 3.6 для группы $\widehat{G}$ сушествует непустое минимальное множество. Несложно заметить, что минимальное множество группы $\widehat{G}$ инвариантно относительно сдвига $\bar{g}(t)=t+1$ и при отображении $\lambda$ образ минимального множества групшы $\widehat{G}$ совпадает с минимальным множеством группы $G$, а прообраз минимального множества группы $G$ совпадает с минимальньм множеством групшы $\widehat{G}$. Поэтому все утверждения теоремы 3.7 следуют из соответствуюших утверждений теоремы 3.1 , примененных к группе $\widehat{G}$.

Весьма важно с помошью более простых подгрупп получить информацию о структуре минимальных множеств исходной группы. Приведем результаты, связьваюшие свойства минимальных множеств исходной группы со свойствами минимальных множеств ее подг рупп.

Лемма 3.3 [11]. Пусть $G \subseteq$ Homeo+ $_{+}(X)$. Если для подгруппьь $\Gamma \subseteq G$ минимальное множество $E(Г)$ непусто и недискретно, то минимальное множество $E(G)$ группь $G$ также непусто и недискретно и, более того, $E(\Gamma) \subseteq E(G)$.

ЛЕмма 3.4 [11]. Пусть $G \subseteq$ Нотео $_{+}(X) . \quad$ Если для нормальной подгруппы $\Gamma \subseteq G$ минимальное множество $E(\Gamma)$ непусто и недискретно, то оно является минимальным множеством исходной группь $G$, m.е. $E(\Gamma)=E(G)$.

Во всех приведенных теоремах (кроме теоремы 3.5 ) доказательство сушествования минимального множества основано на использовании теоремы о фактор-группе и аксиомы выбора и поэтому неконструктивно. 
Существуют специальные группы $G \subseteq \mathrm{Homeo+}_{+}(X)$, важные с точки зрения метрических инвариантов, для которых минимальные множества могут быть конструктивно описаны. К таким группам, в частности, относятся группы $G$ со свойством Fix $G^{s} \neq \varnothing$. (Далее будет использовано обозначение $P(A)$, введенное в $\S 1$.)

Teорема $3.8[9]$. Пусть задана группа $G \subseteq \mathrm{Homeo}_{+}(X)$, для которой Fix $G^{s} \neq \varnothing$. Тогда:

1) для всякого $t \in \operatorname{Fix} G^{s}$ множсество $P(G(t))$ не зависит от точки $t$ (мьи будем обозначать его $\mathbb{P}(G))$;

2) справедливо включение $\mathbb{P}(G) \subseteq \operatorname{Fix} G^{s}$;

3) либо $\mathbb{P}(G)=X$, либо $\mathbb{P}(G)$ - совершенное нигде неплотное подмнохество $\mathbb{X}$, либо $\mathbb{P}(G)=\varnothing ;$

4) если $\mathbb{P}(G) \neq \varnothing$, то группа $G$ имеет единственное недискретное минимальное множество $E(G)$ и $\mathbb{P}(G)$ совпадает с $E(G)$;

5) если $\mathbb{P}(G)=\varnothing$, то все минимальные множсества дискретные, принадлежат множсеству Fix $G^{s}$, а само множество Fix $G^{s}$ состоит из облединения дискретных минимальных множеств.

ДокАЗАтЕльство. В случае $X=\mathbb{R}$ доказательство приведено в [9]. Рассмотрим случай окружности $S^{1}$. Пусть група $\widehat{G}$ и отображения $\bar{g}, \lambda, \pi_{\widehat{G}}$ те же, что и в $\S 1$ (см. предложение 1.1). Несложно заметить, что при отображении $\lambda$ образы минимального множества группы $\widehat{G}$, орбиты $\widehat{G}(t)$ точки $t \in \mathbb{R}$, множества $\mathbb{P}(\widehat{G})$ инвариантны относительно сдвига $\bar{g}(t)=t+1$ и совпадают с минимальным множеством групшы $G$, орбитой $G(\lambda(t))$ точки $\lambda(t) \in S^{1}$, множеством $\mathbb{P}(G)$ соответственно. Верно и обратное. При отоборажении $\lambda$ прообразы минимального множества группы $G$, орбиты $G(t)$ точки $t \in S^{1}$, множества $\mathbb{P}(G)$ совпадают с минимальньм множеством групшы $\widehat{G}$, орбитой $\widehat{G}\left(\lambda^{-1}(t)\right)$ точки $\lambda^{-1}(t) \in \mathbb{R}$, множеством $\mathbb{P}(\widehat{G})$ соответственно.

Тогда утверждение теоремы в случае окружности $S^{1}$ будет следовать из утверждения теоремы для случая $X=\mathbb{R}$, примененной к группе $\widehat{G}$.

Нам остается связать свойства минимальных множеств группы $G \subseteq \operatorname{Homeo}(X)$ и ее нормальной подгруппы $G_{+}$.

Теорема 3.9 [16]. Пусть $G \subseteq \operatorname{Homeo}(X)$. Минимальные множества групnы $G$ и нормальной подгруппь $G_{+}$непусты одновременно. Более того, если минимальное множсество группь $G$ непусто, то справедливо одно из следующих взаимоисключающих утверэсдений:

1) минимальное множсество группь $G$ дискретно и состоит из обтединения не более чем двух дискретных минимальных множсеств нормальной подгруппьи $G_{+}$;

2) минимальное множество группы $G$ недискретно и совпадает с минимальным множеством нормальной подгруппь $G_{+}$.

ДокАЗАтельство. Следует рассмотреть случай $G \neq G_{+}$. Мы знаем, что $G=$ $G_{+} \cup f G_{+}$, где $f-$ некоторьй элемент из $G \backslash G_{+}$. Поэтому если множество $B$ инвариантно относительно подгруппы $G_{+}$, то множество $f(B)$ также будет инвариантньм относительно подгруппы $G_{+}$. Более того, так как $f^{2}(B)=B$, то множество $B \cup f(B)$ будет инвариантным относительно самой группы $G$. 
Пусть для подгрупшы $G_{+}$существует непустое минимальное множество $U$. Как уже отмечалось вьше, множество $f(U)$ инвариантно относительно подгруппы $G_{+}$, а множество $U \cup f(U)$ будет инвариантньм относительно самой группы $G$. Следовательно, для группы $G$ множество $U \cup f(U)$ является минимальньм.

Пусть минимальное множество группы $G$ непусто и $D$ является таким минимальным множеством. В силу инвариантности для любого $g \in G$ его действие на минимальном множестве является гомеоморфизмом. Предположим, что для подгрупшы $G_{+}$не сушествует минимального множества. Фиксируем точку $\bar{t} \in D$. Найдется замкнутое подмножество $\widetilde{D} \subseteq D$, инвариантное относительно подгрупшы $G_{+}$, такое,что точки $\bar{t}, f^{-1}(\bar{t})$ ему не принадлежат. В таком случае точка $\bar{t}$ не будет принадлежать замьканию множества $\widetilde{D} \cup f(\widetilde{D})$ и оно будет собственным замкнутым подмножеством множества $D$, инвариантньм относительно группы $G$. Это противоречит условию минимальности множества $D$, откуда и следует непустота минимального множества для подгрупшы $G_{+}$.

Наконец, если минимальное множество подгрупшы $G_{+}$недискретное, то по теореме 3.1 оно единственное и обозначается через $E\left(G_{+}\right)$. Из инвариантности множества $f\left(E\left(G_{+}\right)\right)$относительно подгруппы $G_{+}$и теоремы 3.1 получим включение $E\left(G_{+}\right) \subseteq$ $f\left(E\left(G_{+}\right)\right)$. Из полученного включения следует, что $f\left(E\left(G_{+}\right)\right) \subseteq f^{2}\left(E\left(G_{+}\right)\right)$. С другой стороны, из инвариантности множества $E\left(G_{+}\right)$относительно подгруппы $G_{+}$и условия $f^{2} \in G_{+}$получим равенство $f^{2}\left(E\left(G_{+}\right)\right)=E\left(G_{+}\right)$. Тогда из полученных включений следует, что $f\left(E\left(G_{+}\right)\right)=E\left(G_{+}\right)$, т.е. минимальные множества групшы $G$ и ее нормальной подгруппы $G_{+}$совпадают.

Если $U$ - дискретное минимальное множество подгрупшы $G_{+}$, то множество $f(U)$ также будет дискретным минимальным множеством для подгруппы $G_{+}$. Множества $U$ и $f(U)$ либо не пересекаются, либо совпадают, а множество $U \cup f(U)$ является минимальным множеством для исходной группы $G$ и состоит из не более чем двух минимальных множеств подгруппы $G_{+} \cdot$

С понятием минимального множества для групшы $G \subseteq \operatorname{Homeo}(X)$ связана некоторая каноническим образом определяемая нормальная подгрупа $H_{G}$, которая играет важную роль в формулировке комбинаторных критериев сушествования метрических инвариантов.

ОПРЕДЕЛЕНИЕ 3.2. Для групшы $G \subseteq \operatorname{Homeo}(X)$ определим нормальную подгруппу $H_{G}$ следующим образом.

1) Если минимальное множество непусто и недискретно, то положим

$$
H_{G}=\left\{h: h \in G_{+}, E(G) \subseteq \operatorname{Fix}\langle h\rangle\right\}
$$

2) Если минимальное множество непусто и дискретно, то положим $H_{G}=G^{s}$.

(По теореме 3.1 из дискретности минимального множества следует непустота множества Fix $G^{s}$. Из непустоты Fix $G^{s}$ следует, что $G^{s}$ является подгруппой, а из замечания 1.1 следует нормальность подгруппы $G^{s}$.)

3) Если минимальное множество пусто, то положим $H_{G}=\langle e\rangle$.

Для гомеоморфизма окружности первый результат об отсутствии минимального множества, изоморфного канторову множеству, был получен Данжуа (см. [27]) для 
циклической группы и известен под названием теоремы Данжуа. Этот же вопрос весьма важен и для групा $G \subseteq \mathrm{Homeo}_{+}(\mathbb{R})[12],[14]-[16]$. Справедливости теоремы Данжуа следует ожидать только для специальных груп. В частности, такая теорема получена для одного важного класса квазисимметрических групп [14].

\section{$\S$ 4. Уточнение теоремы о фактор-группе $G /\left\langle G^{s}\right\rangle$ с использованием свойств минимальных множеств}

Теорема о фактор-группе всего лишш утверждает, что фактор-группа $G /\left\langle G^{s}\right\rangle$ коммутативна. В действительности, важно знать больше о структуре фактор-группы на предмет шикличности либо счетности. Это продиктовано тем, что отмеченные характеристики фактор-групшы тесно связаны как с топологическими свойствами минимальных множеств групшы $G$, так и с другой важной топологической характеристикой $\operatorname{Fix} G^{s}$, выступаюшей критерием сушествования метрического инварианта. Соответствующий результат сформулирован в виде теоремы 4.1. Мы отмечали, что весьма важно уметь выделять более простые подгруппы исходной группы, имеюшие ту же топологическую сложность (то же минимальное множество). Уточнение структуры фактор-группы и позволяет сформулировать предложение о существовании таких простых подгрупп (предложение 4.1).

Далее будет уточнена структура фактор-группы $G /\left\langle G^{s}\right\rangle$ для групшы $G \subseteq$ $\mathrm{Homeo}_{+}(\mathbb{R})$ (теорема 4.1) и сформулирована альтернатива о существовании для такой фактор-групшы абелевой свободной подгруппы с двумя образующими (лемма 4.1, предложение 4.1). Отмеченные уточнения структуры фактор-группы $G /\left\langle G^{s}\right\rangle$ будут приведены и для групшы $G \subseteq \mathrm{Homeo+}_{+}\left(S^{1}\right)$ (теорема 4.2, лемма 4.2, предложение 4.2).

Teоpema 4.1 [9]. Пусть $G \subseteq$ Homeo+ $_{+}(\mathbb{R})$. Тогда фактор-группа $G /\left\langle G^{s}\right\rangle$ коммутативна и изоморфна некоторой подгруппе аддитивной группы $\mathbb{R}$. Более того, для любого $t \in \operatorname{Fix} G^{s}$ множество $P(G(t))$ не зависит от точки $t$, обозначается $\mathbb{P}(G)$ и выполняется одно из следующих взаимоисключающих условий:

1) либо $\operatorname{Fix} G^{s} \neq \varnothing, \mathbb{P}(G)=\mathbb{R}$ (соответственно $\left.\left\langle G^{s}\right\rangle=\langle е\rangle\right)$ и фактор-группа $G /\left\langle G^{s}\right\rangle \cong G$ нечиклическая группа;

2) либо $\operatorname{Fix} G^{s} \neq \varnothing, \mathbb{P}(G)$ является совершенным нигде не плотным подмножеством и фактор-группа $G /\left\langle G^{s}\right\rangle$ нециклическая, но не более чем счетная;

3) либо $\operatorname{Fix} G^{s} \neq \varnothing, \mathbb{P}(G)=\varnothing$ и фактор-группа $G /\left\langle G^{s}\right\rangle$ ичилическая;

4) либо $\operatorname{Fix} G^{s}=\varnothing($ соответственно $\mathbb{P}(G)=\varnothing)$ и фактор-группа $G /\left\langle G^{s}\right\rangle$ тривиальна.

Важньм частным случаем служат группы $G \subseteq \mathrm{Homeo}_{+}(\mathbb{R})$, для которых Fix $G^{s}=$ $\mathbb{R}$, что эквивалентно условию $G^{s}=\langle e\rangle$ (групшы свободно действуюших гомеоморфизмов). По теореме о фактор-группе такие групшы являются коммутативными. В таких группах выделяются специальные подгруппы, необходимые для изучения топологической структуры минимального множества исходной группы $G$. Выделенные подгрупшы алгебраически более простые, но имеют ту же топологическую сложность, что и исходная группа $G$, а именно, минимальные множества выделенных подгрупп совпадают с минимальными множествами исходной групшы. 
Лемма 4.1. Пусть $G \subseteq \mathrm{Homeo}_{+}(\mathbb{R})$ u $\operatorname{Fix} G^{s}=\mathbb{R}$. Тогда для коммутативной нециклической группь $G$ справедлива альтернатива:

1) или группа $G$ содержит свободную абелеву нециклическую подгруппу Г с двумя образующими;

2) или группа $G$ содержит счетную нециклическую подгруппу $Г$, для которой любая конечно-порожденная подгруппа является ииклической.

Более того, минимальные множества группь $G$ и подгруппь Г совпадают.

ДоказАТЕльство. Так как условие $\mathrm{Fix} G^{s}=\mathbb{R}$ эквивалентно условию $\left\langle G^{s}\right\rangle=$ $\langle e\rangle$, то по теореме о фактор-группе (теорема 2.1) группа $G$ изоморфна подгруппе аддитивной группы $\mathbb{R}$, откуда и следуют пш. 1) и 2). Из нецикличности групшы $G$ и подгрупшы Г и теоремы 4.1 следует, что $\mathbb{P}(G) \neq \varnothing$ и $\mathbb{P}(\Gamma) \neq \varnothing$. Поэтому по теореме 3.8 их минимальные множества недискретные, а по лемме 3.4 они совпадают.

Сформулируем альтернативу из леммы 4.1 для произвольных групп $G \subseteq$ $\mathrm{HomeO}_{+}(\mathbb{R})$.

ПрЕДЛОЖЕНИЕ 4.1. Пусть $G \subseteq \mathrm{Homeo}_{+}(\mathbb{R})$. Тогда для коммутативной не чиклической фактор-группь $G /\left\langle G^{s}\right\rangle$ справедлива альтернатива:

1) или фактор-группа $G /\left\langle G^{s}\right\rangle$ содержит свободную абелеву нециклическую подгруппу Г с двумя образующими;

2) или фактор-группа $G /\left\langle G^{s}\right\rangle$ содержит счетную нециклическую подгруппу Г, для которой любая конечно-порожденная подгруппа является ииклической.

Более того, минимальнье множсества группь $G$ и подгруппьи $p_{G}^{-1}(\Gamma)$ совпадают.

ДокАЗАТЕЛЬСТво. По теореме 4.1 коммутативная фактор-группа $G /\left\langle G^{s}\right\rangle$ изоморфна некоторой подгрупе ${ }_{*} G$ аддитивной групшы $\mathbb{R}$. Очевидно, что для нециклической групшы сдвигов ${ }_{*} G$ справедливо условие $\operatorname{Fix} G^{s}=\mathbb{R}$, и она удовлетворяет условиям леммы 4.1. В таком случае, для групшы ${ }_{*} G$ справедлива альтернатива из леммы 4.1 (пп. 1) и 2)), что и доказьвает справедливость пш. 1) и 2) предложения 4.1.

Остается доказать последнее утверждение предложения. Очевидно, что из нецикличности подгруппы $\Gamma$ следует нецикличность подгруппы $p_{G}^{-1}(\Gamma)$ исходной группы $G$. Из нецикличности групшы $G$ и подгруппы $p_{G}^{-1}(\Gamma)$ и теоремы 4.1 следует, что $\mathbb{P}(G) \neq \varnothing$ и $\mathbb{P}\left(p_{G}^{-1}(\Gamma)\right) \neq \varnothing$. Поэтому по теореме 3.8 их минимальные множества недискретные, а по лемме 3.4 они совпадают.

Сформулируем аналоги теоремы 4.1, леммы 4.1 и предложения 4.1 для групп $G \subseteq$ $\mathrm{Homeo}_{+}\left(S^{1}\right)$. Заметим, что группа $\mathrm{Homeo}_{+}(\mathbb{R})$ без кручения, а группа Homeо+ $\left(S^{1}\right)$ с кручением. Поэтому в приводимых ниже теореме, лемме и предложении указания о наличии или отсутствии кручения будут явно присутствовать.

Teоpema 4.2. Пусть $G \subseteq$ Homeo+ $_{+}\left(S^{1}\right)$. Тогда фактор-группа $G /\left\langle G^{s}\right\rangle$ коммутативна и изоморфна некоторой подгруппе аддитивной группь $S^{1}$. Более того, для любого $t \in \operatorname{Fix} G^{s}$ множество $P(G(t))$ не зависит от точки $t$, обозначается $\mathbb{P}(G)$ и выполняется одно из следующих взаимоисключающих условий:

1) либо $\operatorname{Fix} G^{s} \neq \varnothing, \mathbb{P}(G)=S^{1}$ (соответственно $\left\langle G^{s}\right\rangle=\langle$ е $\rangle$ ) и фактор-группа $G /\left\langle G^{s}\right\rangle \cong G$ не является ииклической группой конечного порядка; 
2) либо $\operatorname{Fix} G^{s} \neq \varnothing, \mathbb{P}(G)$ является совершенным нигде не плотнылм подмножеством и фактор-группа $G /\left\langle G^{s}\right\rangle$ не является ииклической группой конечного порядка, при этом она не более чем счетна;

3) либо $\operatorname{Fix} G^{s} \neq \varnothing, \mathbb{P}(G)=\varnothing$ и фактор-группа $G /\left\langle G^{s}\right\rangle$ - ииклическая группа конечного порядка;

4) либо Fix $G^{s}=\varnothing($ соответственно $\mathbb{P}(G)=\varnothing)$ и фактор-группа $G /\left\langle G^{s}\right\rangle$ тривиальна.

ДокАЗАТЕЛЬСТво. Пусть группа $\widehat{G}$ и отображения $\bar{g}, \lambda, \pi_{\widehat{G}}$ те же, что и в $\S 1$ (см. предложение 1.1). Применим к группе $\widehat{G}$ теорему 4.1. Несложно заметить, что при отоборажении $\lambda$ образы минимального множествагруппы $\widehat{G}$, орбиты $\widehat{G}(t)$ точки $t \in \mathbb{R}$, множества $\mathbb{P}(\widehat{G})$ инвариантны относительно сдвига $\bar{g}(t)=t+1$ и совпадают с минимальным множеством группы $G$, орбитой $G(\lambda(t))$ точки $\lambda(t) \in S^{1}$, множеством $\mathbb{P}(G)$ соответственно. Верно и обратное. При отоборажении $\lambda$ прообразы минимального множества группы $G$, орбиты $G(t)$ точки $t \in S^{1}$, множества $\mathbb{P}(G)$ совпадают с минимальньм множеством группы $\widehat{G}$, орбитой $\widehat{G}\left(\lambda^{-1}(t)\right)$ точки $\lambda^{-1}(t) \in \mathbb{R}$, множеством $\mathbb{P}(\widehat{G})$ соответственно. Поэтому для множеств $\operatorname{Fix} G^{s}$ и $\mathbb{P}(G)$ также будут вьполняться четыре взаимоисключающие условия из теоремы.

Остается доказать соответствуюшие условия для фактор-группы $G /\left\langle G^{s}\right\rangle$.

Пусть множества Fix $G^{s}$ и $\mathbb{P}(G)$ удовлетворяют условию 1$)$ или 2 ) теоремы. По теореме 2.1 фактор-група $G /\left\langle G^{s}\right\rangle$ изоморфна некоторой подгруппе аддитивной группы $S^{1}$. Если бы фактор-группа $G /\left\langle G^{s}\right\rangle$ была циклической группой конечного порядка, то вьполнялось бы условие $\mathbb{P}(G)=\varnothing$. Противоречие. Следовательно, в первых двух рассматриваемых случаях фактор-група $G /\left\langle G^{s}\right\rangle$ не является циклической группой конечного порядка.

Пусть множества Fix $G^{s}$ и $\mathbb{P}(G)$ удовлетворяют условию 3$)$ теоремы. Тогда, как отмечалось вьше, множества $\operatorname{Fix} \widehat{G}^{s}$ и $\mathbb{P}(\widehat{G})$ также будут удовлетворять условию 3$)$ теоремы 4.1. По теореме 4.1 фактор-группа $\widehat{G} /\left\langle\widehat{G}^{s}\right\rangle$ - циклическая с образующей $\{g\}$, где через $\{\cdot\}$ обозначается левьй смежньй класс по подгрупе $\left\langle\widehat{G}^{s}\right\rangle$. Так как элеменет $\bar{g}$ принадлежит группе $\widehat{G}$, то найдется натуральное число $n$ такое, что $g^{n}=\bar{g}$.

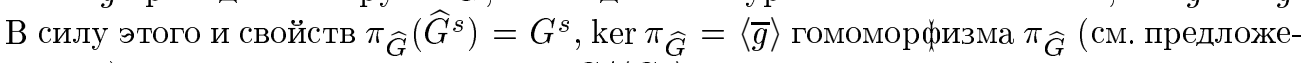
ние 1.1) получим, что фактор-группа $G /\left\langle G^{s}\right\rangle$ является щиклической группой конечного порядка.

Наконец, пусть $\operatorname{Fix} G^{s}=\varnothing$ (условие 4)). Тогда, как отмечалось вьше, множество Fix $\widehat{G}^{s}$ также будет удовлетворять условию 4) теоремы 4.1. По теореме 4.1 фактор-групша $\widehat{G} /\left\langle\widehat{G}^{s}\right\rangle$ тривиальная. В силу этого и свойства $\pi_{\widehat{G}}\left(\widehat{G}^{s}\right)=G^{s}$ гомоморфизма $\pi_{\widehat{G}}$ (см. предложение 1.1 ) получим, что фактор-группа $G /\left\langle G^{s}\right\rangle$ также является тривиальной.

Лемма 4.2. Пусть $G \subseteq$ Homeo $_{+}\left(S^{1}\right)$ u Fix $G^{s}=S^{1}$. Тогда для коммутативной группы $G$, не являющейся чиклической группой конечного порядка, справедлива альтернатива:

1) или группа $G$ содерэит ииклическую подгруппу Г бесконечного порядка;

2) или группа $G$ содержит счетную нециклическую подгруппу $\Gamma$, для которой любая конечно-порожденная подгруппа является чиклической подгруппой конечного порядка. 
Более того, минимальнье множества группь $G$ и подгруппь $\Gamma$ совпадают.

ДокАЗАТЕльство. Так как условие $\mathrm{Fix} G^{s}=S^{1}$ эквивалентно условию $\left\langle G^{s}\right\rangle=$ $\langle e\rangle$, то по теореме о фактор-группе (теорема 2.1) группа $G$ изоморфна подгруппе аддитивной группы $S^{1}$, откуда и следуют пп. 1) и 2). Так как група $G$ и подгруппа $\Gamma$ нециклические групш конечного порядка, то по теореме 4.2 следует, что $\mathbb{P}(G) \neq \varnothing$ и $\mathbb{P}(\Gamma) \neq \varnothing$. Поэтому по теореме 3.8 их минимальные множества недискретные, а по лемме 3.4 они совпадают.

ПРЕДЛОЖЕНИЕ 4.2. Пусть $G \subseteq$ Homeо+ $_{+}\left(S^{1}\right) . \quad$ Тогда для коммутативной фактор-группь $G /\left\langle G^{s}\right\rangle$, не являющейся ииклической группой конечного порядка, справедлива альтернатива:

1) или фактор-группа $G /\left\langle G^{s}\right\rangle$ содержит ииклическую подгруппу Г бесконечного порядка;

2) или фактор-группа $G /\left\langle G^{s}\right\rangle$ содержит счетную нечиклическую подгруппу Г, для которой любая конечно-порожденная подгруппа является ииклической подгруппой конечного порядка.

Более того, минимальные множества группь $G$ и подгруппьи $p_{G}^{-1}(\Gamma)$ совпадают.

ДокАЗАТЕльство. По теореме 4.2 коммутативная фактор-группа $G /\left\langle G^{s}\right\rangle$ изоморфна некоторой подгруппе ${ }_{*} G$ аддитивной группы $S^{1}$. Очевидно, что для ${ }_{*} G$, не являющейся циклической группй вращений конечного порядка, справедливо условие $\operatorname{Fix} G^{s}=S^{1}$ и она удовлетворяет условиям леммы 4.2. Следовательно, для групшы ${ }_{*} G$ справедлива альтернатива из леммы 4.2 (пा. 1) и 2)), что и доказьвает справедливость пп. 1) и 2) предложения 4.2.

Остается доказать последнее утверждение предложения. Так как подгруппа Г не является циклической групой конечного порядка, то подгрупа $p_{G}^{-1}(\Gamma)$ исходной группы $G$ и сама группа $G$ не будут циклическими группами конечного порядка. Тогда по теореме 4.2 следует справедливость условий $\mathbb{P}(G) \neq \varnothing$ и $\mathbb{P}\left(p_{G}^{-1}(\Gamma)\right) \neq \varnothing$. Поэтому по теореме 3.8 их минимальные множества недискретные, а по лемме 3.4 они совпадают.

Лемма 4.1 является определяющей при исследовании вопроса о топологической сопряженности (см. §8) квазисимметрической групшы групе аффинных преобразований прямой $[14]$.

\section{§5. Инвариантные меры}

Вопрос существования инвариантной меры для групшы гомеоморфизмов локально компактного пространства является центральньм в теории Боголюбова-Крылова. Во введении отмечалось, что по истории этого вопроса и важнейшим результатам сушествует исчерпьваюший обзор Д. В. Аносова [2]. Далее мы будем интересоваться группами гомеоморфизмов прямой, для которых сформулируем различные критерии сушествования инвариантной меры. Результаты о существовании инвариантной меры для групп гомеоморфизмов компактного пространства автоматически дают теоремы существования инвариантной меры для груп гомеоморфизмов прямой, являющихся накрытиями гомеоморфизмов окружности. В частности, для таких специальных групп гомеоморфизмов прямой сушествование инвариантной меры следует из 
теоремы Дэйя (см. [20]) о существовании инвариантной меры для дискретных аменабельных групп гомеоморфизмов компактного пространства. Для групп гомеоморфизмов прямой, сохраняющих ориентацию и не являющихся накрытиями гомеоморфизмов окружности, к наиболее сушественньм продвижениям в этой проблематике следует отнести работу Ж.Ф. Планте [32] об эквивалентности вопроса существования инвариантной меры для конечно-порожденных груп условию неэкспоненциального роста орбиты какой-либо точки $t \in \mathbb{R}$. Вместе с тем, теорема Планте не допускает распространения для не конечно-порожденных груп гомеоморфизмов прямой, сохраняющих ориентацию. Поэтому очень важно сформулировать различные критерии сушествования инвариантной меры без априорных предположений о характере групшы. Формулировки и обсуждение теорем Планте и Дэйя будут приведены в $\S 9$, которьй посвящен комбинаторным критериям существования метрических инвариантов.

Центральньм результатом данного параграфа является теорема 5.1, в которой без априорных предположений сфформулирован топологический критерий сушествования инвариантной меры, вскрьваюший природу существования такого метрического инварианта. В некоторых случаях для исследуемых групп проше описать канонически выделенные подгрупш, чем топологические характеристики. Поэтому в разделе 5.1 приводится ряд критериев сушествования инвариантной меры, сфформулированных в терминах канонически выделенных подгрупп. Для инвариантной меры весьма важно описать носитель. Оказывается, что носитель инвариантной меры сосредоточен на минимальных множествах (теорема 5.8). Знания о структуре минимального множества позволяют установить эргодические свойства инвариантной меры (теорема 5.9). Все отмеченные результаты получены для групп гомеоморфизмов $G \subseteq \mathrm{Homeo}_{+}(X)$, сохраняюших ориентацию. Показано, что для произвольной групшы $G \subseteq \operatorname{Homeo}(X)$ сушествование инвариантной меры эквивалентно существованию инвариантной меры для максимальной нормальной подгрупшы $G_{+}$сохраняюших ориентацию гомеоморфизмов (теорема 5.10). Для гомеоморфизма прямой $g \in \widetilde{\text { Нотео }_{+}}\left(S^{1}\right)$, являюшегося накрытием гомеоморфизма окружности, хорошо известен топологический инвариант в виде числа сдвига. Для группы $G \subseteq \widetilde{\text { Homeo+ }_{+}}\left(S^{1}\right)$ число сдвига определяет псевдохарактер. С таким псевдохарактером связан критерий существования инвариантной меры. Существование инвариантной меры для такой группы эквивалентно тому, что псевдохарактер, задаваемьй числом сдвига, является характером (теорема 5.14). В работах Планте определена процедура построения характера по инвариантной мере. Характеры, построенные по любой паре инвариантных мер, являются линейно зависимыми (предложение 5.4). Более того, характеры, задаваемые числом сдвига и какой-либо инвариантной мерой, также оказьваются линейно зависимыми (теоремa 5.15).

Пусть $\Sigma$ есть $\sigma$-алгебра борелевских множеств $X$. Рассмотрим пространство с борелевской (вероятностной в случае $X=S^{1}$ ) мерой, конечной на компактах, т.е. тройку $(X, \Sigma, \mu)$.

ОПРЕДЕЛЕНИЕ 5.1. Борелевская (вероятностная в случае $X=S^{1}$ ) мера $\mu$, конечная на компактах, назьвается инвариантной относительно групшы $G \subseteq \operatorname{Homeo}(X)$, если для любых $g \in G, B \in \Sigma$ вьполняется условие $\mu\left(q^{-1}(B)\right)=\mu(B)$.

Далее для груп $G \subseteq \mathrm{Homeo}_{+}(X)$ будет сформулирован основной топологический критерий существования инвариантной меры (теорема 5.1), а также критерии, 
использующие метрические и алгебраические свойства канонических подгрупп (теоремы 5.2-5.4). Для таких групп имеют место и иные критерии существования инвариантной меры, основанные на свойствах конечно-порожденных подгрупп (теоремы 5.5-5.7). Одним из важных вопросов для инвариантных мер является описание их носителей (теорема 5.8). Не менее важным является описание всего множества инвариантных мер. В этой связи сформулированы критерий (теорема 5.9) и признаки (предложения $5.1,5.2$ ) строгой эргодичности групшы $G \subseteq \mathrm{Homeo}_{+}(X)$. Описана связь между инвариантными мерами группы $G \subseteq \operatorname{Homeo}(X)$ и ее нормальной подгруппы $G_{+}$(теорема 5.10), а также их свойствами строгой эргодичности (теорема 5.11). Отмечено, что существование инвариантной меры является достаточным условием сушествования непустого минимального множества (предложение 5.3).

Для груп $G \subseteq$ Homeo+ $_{+}(\mathbb{R})$ с инвариантной мерой $\mu$ определяется характер $\tau_{\mu, G}$ (предложение 5.4). В терминах характера $\tau_{\mu, G}$ переформулирована теорема о строгой эргодичности (теорема 5.12). Для любого гомеоморфизма $g \in \widehat{\text { Homeо }_{+}}\left(S^{1}\right)$ сушествует хорошо известньй топологический инвариант $\tau(g)$, назьваемьй числом сдвига $(\bmod 1$; оно назьвается числом врашения). Отображение, определяемое числом сдвига, является псевдохарактером (теорема 5.13). Для группы $G \subseteq \widetilde{\text { Homeo+ }_{+}}\left(S^{1}\right)$ устанавливается соответствие между свойствами ограничения $\tau_{G}=\left.\tau\right|_{G}$ псевдохарактера $\tau$ на группу $G$ и вопросом сушествования инвариантной меры $\mu$, а также характером $\tau_{\mu, G}$ (теоремы $\left.5.14,5.15\right)$. Сдвиг $\bar{g}(t) \equiv t+1$ принадлежит центру групшы $\widehat{\mathrm{Homeo}}_{+}\left(S^{1}\right)$. Вследствие этого для любой групшы $G \subseteq \widetilde{\text { Нотео }_{+}}\left(S^{1}\right)$ ее метрические свойства совпадают с метрическими свойствами более широкой группы $\langle G, \bar{g}\rangle$ (предложение 5.5). Для циклической группы $G=\langle g\rangle$ дается детальное описание структуры коммутативной групшы $\langle g, \bar{g}\rangle$ и ее связь с характером значения числа сдвига $\tau(g)$ (теорема 5.16).

Другой способ построения характера для групп $G \subseteq \mathrm{Homeo}_{+}(\mathbb{R})$ связан с существованием нормальной подгруппы $\Gamma \subseteq G$ с инвариантной мерой $\mu$ и нетривиальньм характером $\tau_{\mu, \Gamma}[33]$. Поэтому дается описание таких подгрупп $\Gamma$ (лемма 5.1). Уточняется структура груп $G \subseteq \mathrm{Homeo}_{+}(\mathbb{R})$, допускаюших построение нетривиального характера вторым способом (предложение 5.6), а также указано на достаточность такого условия для сушествования непустого минимального множества для исходной групшы (следствие 5.2).

Для групп с инвариантной мерой уточняется структура канонической подгруппы $H_{G}$ (лемма 5.2).

5.1 Топологические и алгебраические критерии существования инвариантной меры. Приведем наиболее важньй топологический критерий сушествования инвариантной меры.

Теорема 5.1 [9]. Пусть $G \subseteq$ Homeo+ $_{+}(X)$. Тогда следующие утверәсдения әквивалентны:

1) существует борелевская (вероятностная в случае $X=S^{1}$ ) мера $\mu$, конечная на компактах и инвариантная относительно группь $G$;

2) мнохсество Fix $G^{s}$ непусто.

ДокАЗАТЕЛЬСтво. В случае $X=\mathbb{R}$ доказательство приведено в [9]. Рассмотрим случай окружности $X=S^{1}$. Пусть группа $\widehat{G}$ и отображения $\bar{g}, \lambda, \pi_{\widehat{G}}$ те же, что и в $\S 1$ 
(см. предложение 1.1). Несложно заметить, что множества Fix $\widehat{G}^{s}$ и Fix $G^{s}$ непусты одновременно и при отображении $\lambda$ образ множества Fix $\widehat{G}^{s}$ совпадают с множеством Fix $G^{s}$. С другой стороны, для групшы $\widehat{G}$ инвариантная борелевская мера, конечная на компактах, существует тогда и только тогда, когда для групшы $G$ существует инвариантная вероятностная мера. Тогда утверждение теоремы в случае окружности $S^{1}$ будет следовать из утверждения теоремы для случая $X=\mathbb{R}$, примененной к групе $\widehat{G}$.

В работе [11] была сформулирована теорема существования инвариантной меры, использующая топологические и алгебраические характеристики групшы $G \subseteq$ Homeо $_{+}(\mathbb{R})$. Приведем ее формулировку с некоторыми очевидньми модификациями и полным доказательством.

Tеорема 5.2. Пусть $G \subseteq$ Нотео+ $_{+}(\mathbb{R})$. Тогда следующие утверждения эквивалентны:

а) существует борелевская мера $\mu$, конечная на компактах и инвариантная относительно группы $G$;

б) существует точка $t \in \mathbb{R}$, для которой $\sup _{g \in\left\langle G^{s}\right\rangle}|g(t)|<+\infty$;

в) справедливо хотя бь одно из условий: (1) фактор-группа $G /\left\langle G^{s}\right\rangle$ нетривиальная; (2) существует непустое дискретное минимальное множесmвo;

г) справедливо хотя бь одно из условий: (1) $G=C_{G} \neq\left\langle G_{\infty}^{s}\right\rangle=G_{\infty}^{s} ;(2)$ существует непустое дискретное минимальное множество.

ДокАЗАТЕльСтво. Пусть справедливо утверждение а). По теореме 5.1 справедливо условие $\operatorname{Fix} G^{s} \neq \varnothing$ и для любой точки $t \in \operatorname{Fix} G^{s}$ будет вьполняться неравенство из п. б).

Пусть справедливо условие б). В таком случае $\operatorname{Fix} G^{s} \neq \varnothing$, иначе по теореме 4.1 (п. 4)) $G=\left\langle G^{s}\right\rangle$, а по лемме 3.2 орбита $G(t)$ неограничена, что противоречит предположению б). Из условия $\mathrm{Fix} G^{s} \neq \varnothing$ следует, что сушествует непустое минимальное множество. Если минимальное множество недискретное, то (см. теоремы $3.8,4.1)$ фактор-группа $G /\left\langle G^{s}\right\rangle$ нетривиальная, что и доказьвает п. в).

Пусть справедливо условие в). Тогда из условия (1) п. в) и леммы 2.3 следует условие (1) п. г).

Остается показать, что из г) следует а). Из г) следует, что Fix $G^{s} \neq \varnothing$, иначе (см. п. 4) теоремы 4.1) фактор-группа $G /\left\langle G^{s}\right\rangle$ будет тривиальной, что противоречит предположению г). Поэтому условие а) следует из теоремы 5.1.

Для групп $G \subseteq \widetilde{\text { Нотео }_{+}}\left(S^{1}\right)$ выполняется условие $G_{\infty}^{s}=G^{s}$ (см. замечание 2.1$)$, и поэтому для них справедливо условие $G=C_{G}$. В силу этого для указанного класса групп теорема 5.2 может быть уточнена.

Teоpema 5.3. Пусть $G \subseteq \widetilde{\text { Нотео }_{+}}\left(S^{1}\right)$. Тогда следующие утверждения эквивалентны:

а) существует борелевская мера $\mu$, конечная на компактах и инвариантная относительно группь $G$;

б) существует точка $t \in \mathbb{R}$, для которой $\sup _{g \in\left\langle G^{s}\right\rangle}|g(t)|<\infty$; 
в) справедливо хотя бь одно из условий: (1) фактор-группа $G /\left\langle G^{s}\right\rangle$ нетривиальная; (2) существует непустое дискретное минимальное множество;

г) $\left\langle G_{\infty}^{s}\right\rangle=G_{\infty}^{s}$.

ДокАЗАТЕльСТво. Эквивалентность условий а), б), в) следует из теоремы 5.2.

Напомним, что для рассматриваемых групп вьполняется условие $G^{s}=G_{\infty}^{s}$ и поэтому $G=C_{G}$. По теореме 3.6 сушествует непустое минимальное множество. Более того, если $\left\langle G^{s}\right\rangle=G^{s}$ и фактор-групша $G /\left\langle G^{s}\right\rangle$ тривиальная $\left(\right.$ соответственно $\left.G=\left\langle G^{s}\right\rangle\right)$, то по следствию 3.1 вьполняется условие Fix $G^{s} \neq \varnothing$, а по теоремам 4.1 и 3.8 минимальное множество дискретное. Следовательно, для рассматриваемого класса групп условие г) теоремы 5.2 эквивалентно условию г) теоремы 5.3, что и завершает доказательство.

Дадим аналог теоремы 5.3 для груп, действуюших на окружности.

Теорема 5.4. Пусть $G \subseteq$ Нотео+ $_{+}\left(S^{1}\right)$. Тогда следующие утверждения әквивалентны:

а) существует вероятностная мера $\mu$, инвариантная относительно груп$n$ bl $G$;

б) справедливо хотя бь одно из условий: (1) фактор-группа $G /\left\langle G^{s}\right\rangle$ нетривиальная; (2) существует непустое дискретное минимальное множество;

в) $\left\langle G^{s}\right\rangle=G^{s}$.

ДокАЗАТЕльство. Пусть группа $\widehat{G}$ и отображения $\bar{g}, \lambda, \pi_{\widehat{G}}$ те же, что и в $\S 1$ (см. предложение 1.1). Несложно заметить, что условия $\left\langle G^{s}\right\rangle=G^{s}$ и $\left\langle\widehat{G}^{s}\right\rangle=\widehat{G}^{s}$ вьполняются одновременно, а по предложению 1.1 при гомоморфизме $\pi_{\widehat{G}}$ образ $\widehat{G}^{s}$ совпадает с $G^{s}$. С другой стороны, для групшы $\widehat{G}$ инвариантная борелевская мера, конечная на компактах, существует тогда и только тогда, когда для группы $G$ сушествует инвариантная вероятностная мера. Более того, минимальные множества групп $G$ и $\widehat{G}$ дискретны либо недискретны одновременно.

В таком случае утверждение теоремы будет следовать из утверждения теоремы 5.3, примененной к группе $\widehat{G}$.

Сфформулируем иной критерий существования инвариантной меры, использующий свойства конечно-порожденных подгрупп.

Теорема $5.5[11]$. Пусть $G \subseteq$ Homeo $_{+}(\mathbb{R})$. Для существования борелевской мерь, конечной на компактах и инвариантной относительно группь $G$, необходимо и достаточно, чтобь:

а) для любой конечно-порожденной подгруппь $\Gamma \subseteq G$ существовала борелевская мера, конечная на компактах и инвариантная относительно nодгруппь $\Gamma$;

б) существовало натуральное число $n$ такое, что для любой конечно-порожсденной подгруппь $\Gamma \subseteq G$ выполняется условие $[-n, n] \cap \operatorname{Fix} \Gamma^{s} \neq \varnothing$.

Для групп $G \subseteq \widetilde{\text { Ноmео }_{+}}\left(S^{1}\right)$ теорема 5.5 может быть уточнена, так как для таких груп условие б) автоматически вьполняется. 
Теорема 5.6. Пусть $G \subseteq \widetilde{\text { Hотео }_{+}}\left(S^{1}\right)$. Для существования борелевской ме$p$ в, конечной на компактах и инвариантной относительно группы $G$, необходимо и достаточно, чтобы для любой конечно-порожденной подгруппь $\Gamma \subseteq G$ существовала борелевская мера, конечная на компактах и инвариантная относительно подгруппьг Г.

ДоКАЗАТЕльство. Если для подгруппы $\Gamma \subseteq G$ выполняется условие Fix $\Gamma^{s} \neq \varnothing$, то в силу инвариантности множества $\operatorname{Fix} \Gamma^{s}$ относительно гомеоморфизма $\bar{g}(t)=t+1$ справедливо условие $[0,1] \cap \mathrm{Fix}^{s} \neq \varnothing$. В таком случае доказательство следует из теоремы 5.5 .

Сформулируем аналог теоремы 5.6 для груп, действуюших на окружности.

Теорема 5.7. Пусть $G \subseteq$ Homeо+ $_{+}\left(S^{1}\right)$. ДЛя существования вероятностной меры, инвариантной относительно группь $G$, необходимо и достаточно, чтобь для любой конечно-порожденной подгруппь $\Gamma \subseteq G$ существовала вероятностная мера, инвариантная относительно подгруппь $\Gamma$.

ДокаЗАТЕльство. Пусть группа $\widehat{G}$ и отображения $\bar{g}, \lambda, \pi_{\widehat{G}}$ те же, что и в $\S 1$ (см. предложение 1.1). Несложно заметить, что для гомоморфизма $\pi_{\widehat{G}}$ прообразом конечно-порожденных подгрупп являются конечно-порожденные подгрупшы. С другой стороны, для групы $\widehat{G}$ инвариантная борелевская мера, конечная на компактах, существует тогда и только тогда, когда для группы $G$ существует инвариантная вероятностная мера. Поэтому утверждение теоремы будет следовать из утверждения теоремы 5.6, примененной к группе $\widehat{G}$.

5.2. Эргодические свойства инвариантной меры. Для изучения свойств инвариантной меры следует описать ее носитель. Этому будут посвящены результаты данного раздела. Будут сфформулированы результаты о строгой эргодичности, а также о взаимосвязи инвариантных мер группы $G$ и ее нормальной подгруппы $G_{+}$.

Tеорема 5.8 [9]. Пусть $G \subseteq$ Homeо+ $_{+}(X)$ и существует борелевская (вероятностная в случае $X=S^{1}$ ) мера $\mu$, конечная на компактах и инвариантная относительно группь $G$. Тогда:

1) для носителя меры $\mu$ имеет место включение $\operatorname{supp} \mu \subseteq \operatorname{Fix} G^{s}$;

2) если $\mathbb{P}(G) \neq \varnothing$, то $\operatorname{supp} \mu=\mathbb{P}(G)=E(G)$; более того, в этом случае мера $\mu$ непрерывна;

3) если $\mathbb{P}(G)=\varnothing$, то $\operatorname{supp} \mu$ состоит из некоторого обгединения дискретных минимальных множеств.

ДокАЗАтЕльство. В случае $X=\mathbb{R}$ доказательство приведено в [9]. Рассмотрим случай окружности $X=S^{1}$. Пусть група $\widehat{G}$ и отображения $\bar{g}, \lambda, \pi_{\widehat{G}}$ те же, что и в $\S 1$ (см. предложение 1.1). Несложно заметить, что множества Fix $\widehat{G}^{s}$ и Fix $G^{s}$ непусты одновременно и при отображении $\lambda$ образы множеств $\operatorname{Fix} \widehat{G}^{s}, \mathbb{P}(\widehat{G})$ совпадают с множествами $\operatorname{Fix} G^{s}, \mathbb{P}(G)$ соответственно. Тогда утверждение теоремы в случае окружности $S^{1}$ будет следовать из утверждения теоремы для случая $X=\mathbb{R}$, примененной к группе $\widehat{G}$. 
ОПРЕДЕЛЕНИЕ 5.2. Группа гомеоморфизмов $G \subseteq \operatorname{Homeo~}(X)$ назьвается строго эргодичной, если сушествует борелевская (вероятностная в случае $X=S^{1}$ ) мера, конечная на компактах и инвариантная относительно групшы $G$, и для любой пары $\mu_{1}, \mu_{2}$ борелевских (вероятностных) мер, конечных на компактах и инвариантных относительно группы $G$, справедливо соотношение $\mu_{1}=\lambda \mu_{2}, \lambda \subseteq \mathbb{R}^{+}\left(\mu_{1}=\mu_{2}\right)$.

Сформулируем теорему о строгой эргодичности. Ее доказательство основано на свойстве строгой эргодичности для нециклической групшы сдвигов на прямой.

Tеорема 5.9 [9]. Пусть $G \subseteq$ Homeо+ $_{+}(X)$ и существует борелевская (вероятностная в случае $X=S^{1}$ ) мера, конечная на компактах и инвариантная относительно группь $G$. Группа $G$ является строго эргодичной тогда и только тогда, когда выполняется одно из взаимоисключающих условий:

1) $\mathbb{P}(G) \neq \varnothing$;

2) $\mathbb{P}(G)=\varnothing$, а множество $\mathrm{Fix} G^{s}$ состоит из одного непустого дискретного минимального мноэества.

ДокАЗАТЕльство. В случае $X=\mathbb{R}$ доказательство приведено в [9]. В случае окружности $X=S^{1}$ доказательство этой теоремы дословно повторяет доказательство теоремы 5.8 .

Сформулируем одно важное достаточное условие строгой эргодичности.

ПРЕДЛОЖЕНИЕ 5.1. Пусть $G \subseteq \mathrm{Homeo}_{+}(\mathbb{R})$. Для строгой әргодичности групnы $G$ достаточно, чтобы фактор-группа $G /\left\langle G^{s}\right\rangle$ не была ииклической.

ДокАЗАТЕЛЬСТВо. По теореме 4.1 для такой групшы справедливы условия Fix $G^{s} \neq \varnothing$ и $\mathbb{P}(G) \neq \varnothing$. Тогда по теоремам 5.1 и 5.9 группа $G$ строго эргодичная.

Сформулируем аналог предложения 5.1 для групп $G \subseteq \mathrm{Homeo}_{+}\left(S^{1}\right)$.

ПРЕДЛОЖЕНИЕ 5.2. Пусть $G \subseteq$ Homeо $_{+}\left(S^{1}\right)$. Для строгой эргодичности группь $G$ достаточно, чтобь фактор-группа $G /\left\langle G^{s}\right\rangle$ не бъла чиклической группой конечного порядка.

ДокАЗАТЕльСТВо. По теореме 4.2 для такой групшы справедливы условия Fix $G^{s} \neq \varnothing$ и $\mathbb{P}(G) \neq \varnothing$. Тогда по теореме 5.9 группа $G$ строго эргодичная.

Теорема 5.8 и теорема о строгой эргодичности (теорема 5.9) описьвают все инвариантные меры и их носители. В частности, если минимальное множество недискретное, то инвариантная мера единственная (с точностью до растяжения), а ее носитель совпадает с единственньм минимальным множеством. При изучении спектральных свойств группы операторов сдвига, индуцированных группой гомеоморфизмов прямой, возникает проблема описания всех инвариантных множеств (а не только носителей инвариантных мер) [3]. Оказывается, что инвариантные множества (не обязательно замкнутые) могут иметь сколь угодно сложную природу. В работе [3] построен пример коммутативной группы $\langle g, \bar{g}\rangle$, где $g \in \widehat{\text { Ноmео }_{+}}\left(S^{1}\right), \bar{g}(t)=t+1$, для которой минимальное множество совпадает со всей прямой и существует инвариантное множество (естественно, не замкнутое) неполной лебеговой меры.

Теперь мы в состоянии описать связь между инвариантньми мерами групшы $G \subseteq$ $\operatorname{Homeo}(X)$ и ее нормальной подгруппы $G_{+}$. 
Теорема 5.10. Пусть $G \subseteq \operatorname{Homeo}(X)$. Для группь $G$ инвариантная мера (вероятностная мера в случае $X=S^{1}$ ) существует тогда и только тогда, когда существует инвариантная мера (вероятностная мера) для нормальной nодгруппьи $G_{+}$.

ДоКАЗАТЕльство. В одну сторону доказательство очевидно. Пусть для нормальной подгрупшы $G_{+}$сушествует инвариантная мера $\mu$. Следует рассмотреть случай $G \neq G_{+}$. Мы знаем, что $G=G_{+} \cup f G_{+}$, где $f$ - некоторьй элемент из $G \backslash G_{+}$.

Пусть минимальное множество подгрупшы $G_{+}$дискретное. Обозначим его через $D$. Тогда дискретное множество $f(D)$ также является минимальным множеством подгрупшы $G_{+}$, и по теореме 3.9 объединение $D \cup f(D)$ будет дискретным минимальным множеством самой группы $G$. По дискретному минимальному множеству групшы $G$ очевидньм образом строится мера, инвариантная относительно $G$.

Пусть минимальное множество нормальной подгрупшы $G_{+}$недискретное. Если $q$ гомеоморфизм, то определим меру $q_{*} \mu$ по следуюшему правилу: для любого борелевского множества $B$ должно вьполняться условие $q_{*} \mu(B)=\mu\left(q^{-1}(B)\right)$. Из нормальности подгруппы $G_{+}$следует, что для любого элемента $g \in G$ мера $g_{*} \mu$ также инвариантна относительно подгрупшы $G_{+}$. Так как минимальное множество подгруппы $G_{+}$недискретное, то по теореме $5.9 g_{*} \mu=d_{g} \mu$, где $d_{g}>0$. Очевидно, что для элементов $g \in G_{+}$значение $d_{g}$ равно единице. Для элемента $g \neq G_{+}$справедливо условие $g^{2} \in G_{+}$, поэтому должно вьполняться равенство $d_{g}^{2}=1$. Следовательно, для любого элемента $g \in G$ также должно выполняться условие $d_{g}=1$, откуда и следует инвариантность меры $\mu$ относительно исходной группы $G$.

Опишем связь между свойствами строгой эргодичности группы $G \subseteq \operatorname{Homeo}(X)$ и нормальной подгруппы $G_{+}$.

Теорема 5.11. Пусть $G \subseteq \operatorname{Homeo}(X)$ и существует борелевская (вероятностная в случае $X=S^{1}$ ) мера $\mu$, конечная на компактах и инвариантная относительно группь $G$. Если подгруппа $G_{+}$строго әргодичная, то и группа $G$ будет строго әргодичной. Если минимальное множество недискретное, то группа $G$ и подгруппа $G_{+}$строго эргодичны.

ДоказАТЕльство. Первое утверждение очевидно. Докажем второе утверждение. По теореме 3.9 минимальное множество подгруппы $G_{+}$также не дискретное. Тогда по теореме 5.9 подгруппа $G_{+}$будет строго эргодичной, откуда и следует строгая эргодичность исходной группы $G$.

Сформулируем одно простое утверждение о минимальных множествах. Напомним, что для групшы $G \subseteq \widehat{\operatorname{Homeo}}\left(S^{1}\right)\left(G \subseteq \operatorname{Homeo}\left(S^{1}\right)\right)$ минимальное множество непусто.

ПРЕДЛОЖЕНИЕ 5.3. Для группы $G \subseteq \operatorname{Homeo(}(\mathbb{R})$ с инвариантной борелевской мерой, конечной на компактах, минимальное множество непусто.

ДокАЗАТЕЛЬСтво. В силу теоремы 3.9 достаточно рассмотреть группы $G \subseteq$ $\mathrm{Homeo}_{+}(\mathbb{R})$. Но для таких групп по теореме 5.1 существование инвариантной меры эквивалентно условию $\mathrm{Fix} G^{s} \neq \varnothing$. Тогда непустота минимального множества следует из теоремы 3.8 . 
5.3. Число сдвига и число вращения. Характеры, псевдохарактеры, квазихарактеры. При исследовании структуры группы $Q$ весьма важно иметь информацию о группе гомоморфизмов (характеров) $\operatorname{Hom}(Q, \mathbb{R})$, которая образует векторное пространство над полем действительных чисел. Часто оказывается полезным исследование объектов из более общих линейных пространств псевдогомоморфизмов (псевдохарактеров) и квазигомоморфизмов (квазихарактеров).

ОПреДЕлЕниЕ 5.3 [21]. Пусть $G$ - абстрактная группа. Отображение $f: G \rightarrow \mathbb{R}$ называется квазихарактером, если существует константа $L_{f}>0$ такая, что для любых $g_{1}, g_{2} \in G$ справедливо условие

$$
\left|f\left(g_{1} g_{2}\right)-f\left(g_{1}\right)-f\left(g_{2}\right)\right|<L_{f} .
$$

Отображение $f: G \rightarrow \mathbb{R}$ называется псевдохарактером, если оно является квазихарактером и для любых $g \in G, n \in \mathbb{Z}$ вьполняется равенство $f\left(g^{n}\right)=n f(g)$.

В дальнейшем для группы $G$ будем пользоваться обозначениями $X(G)=$ $\operatorname{Hom}(G, \mathbb{R}), P X(G), Q X(G)$ характеров, псевдохарактеров и квазихарактеров соответственно. Очевидно, что $X(G) \subseteq P X(G) \subseteq Q X(G)$.

В работе [21] для абстрактных групп изучается взаимосвязь пространств $X(G)$, $P X(G), Q X(G)$ с ограниченными когомологиями. В частности, там приводится результат, в силу которого для аменабельных групп из существования нетривиального квазихарактера следует сушествование нетривиального характера.

Один из способов построения характера для груп $G \subseteq \mathrm{Homeo}_{+}(\mathbb{R})$ связан с наличием инвариантной меры. Следуя работе [33], опишем процедуру построения характера для групшы $G \subseteq \mathrm{Homeo+}_{+}(\mathbb{R})$ с инвариантной мерой.

ПРЕДЛОЖЕНИЕ 5.4 [33]. Пусть $G \subseteq$ Hотео $_{+}(\mathbb{R})$, а мерой, конечной на компактах и инвариантной относительно группь $G$. Тогда для любого $g \in G$ число

$$
\tau_{\mu, G}(g)= \begin{cases}\mu([t, g(t))), & \text { если } g(t) \geqslant t, \\ -\mu([g(t), t)), & \text { если } g(t)<t,\end{cases}
$$

не зависит от точки $t \in \mathbb{R}$, а отображсение $\tau_{\mu, G}: G \rightarrow \mathbb{R}$ является характером. Более того, если $\mu_{1}, \mu_{2}$ - две борелевские меры, конечные на компактах и инвариантные относительно группь $G$, то $\tau_{\mu_{1}, G}=\lambda \tau_{\mu_{2}, G}, \lambda \in \mathbb{R}_{+}$.

ЗАмЕчАниЕ 5.1. В случае существования инвариантной меры множество $G^{s}$ образует группу, а из определения характера $\tau_{\mu, G}$ следует, что $\operatorname{ker} \tau_{\mu, G}=G^{s}$. Поэтому фактор-группа $G /\left\langle G^{s}\right\rangle$ изоморфна образу $\operatorname{Im} \tau_{\mu, G}$ характера $\tau_{\mu, G}$.

Используя характер $\tau_{\mu, G}$, мы можем несколько переформулировать теорему о строгой эргодичности групшы $G \subseteq \mathrm{Homeo}_{+}(\mathbb{R})$ (теорема 5.9).

Tеорема 5.12 [10]. Пусть $G \subseteq$ Homeo+ $_{+}(\mathbb{R})$ и существует борелевская мера $\mu$, конечная на компактах и инвариантная относительно группь $G$. Группа $G$ является строго эргодичной тогда и только тогда, когда вьполняется одно из условий:

1) образ $\operatorname{Im} \tau_{\mu, G}$ всюду плотен в $\mathbb{R}$;

2) образ $\operatorname{Im} \tau_{\mu, G}$ - дискретное множсество, а мнохсество Fix $G^{s}$ состоит из одного дискретного минимального множества. 
Строгая эргодичность групшы $G \subseteq \mathrm{Homeo+}_{+}(\mathbb{R})$ в предположении 1) теоремы 5.12 была доказана в работе [33].

Для группы $\widetilde{\mathrm{Homeo}_{+}}\left(S^{1}\right)$ сушествует хорошо известная процедура построения псевдохарактера, не связанная с наличием инвариантной меры.

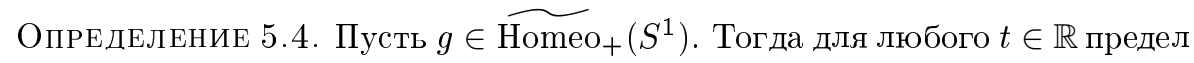

$$
\lim _{n \rightarrow \infty} \frac{g^{n}(t)}{n}
$$

существует и не зависит от $t$. Этот предел назьвается иислом сдвига и обозначается $\tau(g)$. Если $f \in$ Ноmeо $_{+}\left(S^{1}\right)$, a $g \in \widehat{\text { Нотео }_{+}}\left(S^{1}\right)$ - произвольньй элемент, являюшийся накрытием гомеоморфизма $f$, то корректно определено число вращения $\rho(f)=\tau(g) \bmod 1$ гомеоморфизма $f$.

В работе [19] для всей групы $\overparen{\text { Нотео }_{+}}\left(S^{1}\right)$ приводится результат о структуре пространства псевдохарактеров.

Теорема 5.13 [19]. Для группь $\widetilde{\text { Homeo }_{+}}\left(S^{1}\right)$ отображсение $\tau: \widetilde{\text { Нотео }_{+}}\left(S^{1}\right) \rightarrow \mathbb{R}$, порожденное числом сдвига $\tau(g), g \in \widehat{\text { Нотео }_{+}}\left(S^{1}\right)$, является псевдохарактером. Более того, пространство псевдохарактеров $P X\left(\widetilde{\mathrm{HomeO}_{+}}\left(S^{1}\right)\right)$ одномерное.

Для групп $G \subseteq \widehat{\text { Нотео }_{+}}\left(S^{1}\right)$, в дополнение к теореме 5.3, мы можем сформулировать новый критерий существования инвариантной меры, использующий число сдвига $\tau(g)$ гомеоморфизма $g \in G$.

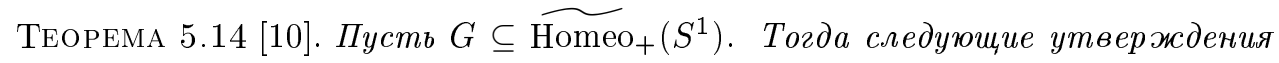
әквивалентны:

1) существует борелевская мера $\mu$, конечная на компактах и инвариантная относительно группь $G$;

2) псевдохарактер $\tau_{G}: G \rightarrow \mathbb{R}$, являющийся ограничением псевдохарактера $\tau: \overbrace{\text { Hотео }_{+}}\left(S^{1}\right) \rightarrow \mathbb{R}$ на подгруппу $G$, является характером.

В силу теорем $5.1,5.3$ и 5.14 для групп $G \subseteq \widetilde{\text { Нотео }_{+}}\left(S^{1}\right)$ мы имеем эквивалентность следуюших трех утверждений: $\mathrm{Fix} G^{s} \neq \varnothing$; псевдохарактер $\tau_{G}$ является характером; объединение стабилизаторов $G^{s}$ образует группу. В работе [40] для групп $G \subseteq \widetilde{\text { Homeо }_{+}}\left(S^{1}\right)$, не содержаших свободных подполугрупп с более чем одной образующей, доказана справедливость каждого из трех утверждений, хотя вопрос об их эквивалентности (соответственно их эквивалентности существованию инвариантной меры) не исследовался.

Для груп $G \subseteq \widetilde{\text { Нотео }_{+}}\left(S^{1}\right)$ с инвариантной мерой $\mu$ существует взаимосвязь между характерами $\tau_{G}$ и $\tau_{\mu, G}$.

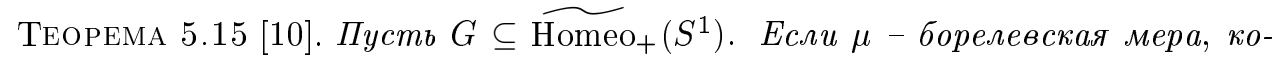
нечная на компактах и инвариантная относительно группь $G$, то $\tau_{G}=\lambda \tau_{\mu, G}$, $\lambda \in \mathbb{R}_{+}$.

Далее мы попытаемся установить связь между числом сдвига $\tau(g)$ гомеоморфизма $g \subseteq \widetilde{\text { Нотео }_{+}}\left(S^{1}\right)$ и структурой коммутативной группы $\mathscr{J}_{g}=\langle g, \bar{g}\rangle$, где $\bar{g}(t)=$ 
$t+1$. Для этого установим взаимосвязь между метрическими свойствами групп $G \subseteq$ $\mathrm{Homeo}_{+}\left(S^{1}\right)$ и $\langle G, \bar{g}\rangle$.

ПРЕДЛОЖЕНИЕ 5.5 [10]. Пусть $G \subseteq \widetilde{\text { Ноmео }_{+}}\left(S^{1}\right)$. Борелевская мера, конечная на компактах и инвариантная относительно группь $G$, существует тогда $u$ только тогда, когда существует борелевская мера, конечная на компактах и инвариантная относительно группьи $\langle G, \bar{g}\rangle$.

Очевидно, что для любой циклической группы $\langle g\rangle, g \in \mathrm{Homeo}_{+}(\mathbb{R})$, существует инвариантная борелевская мера, конечная на компактах. Поэтому для любого $g \in$ $\widehat{\text { Ноmeо }}_{+}\left(S^{1}\right)$ из предложения 5.4 можно извлечь следствие относительно коммутативной групшы $\mathscr{J}_{g}=\langle g, \bar{g}\rangle$.

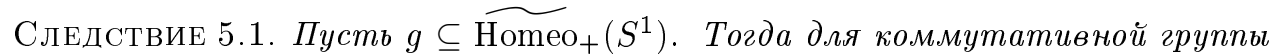
$\mathscr{J}_{g}=\langle g, \bar{g}\rangle$ существует инвариантная борелевская мера, конечная на компак$\max$.

В действительности, можно получить дополнительную информацию как о структуре группы $\mathscr{J}_{g}$, так и о взаимосвязи структуры грушы $\mathscr{J}_{g}$ и числа сдвига $\tau(g)$ в виде следствия из теоремы Пуанкаре (теорема Пуанкаре приведена в [19]).

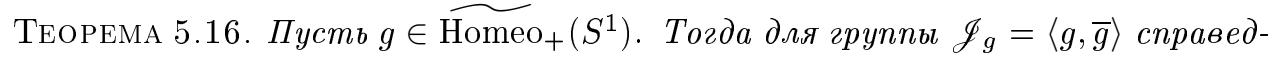
лива альтернатива:

1) либо $\operatorname{Fix} \mathscr{J}_{g}^{s}=\mathbb{R}$ (что әквивалентно условию $\mathscr{J}_{g}^{s}=\langle e\rangle$, соответственно, әруппа $\mathscr{J}_{g}$ состоит из свободно действующих гомеоморфизмов);

2) либо $\mathrm{Fix} \mathscr{J}_{g}^{s} \neq \mathbb{R}$, а фактор-группа $\mathscr{J}_{g} / \mathscr{J}_{g}^{s}$ и подгруппа $\mathscr{J}_{g}^{s}$ ииклические. Более того, число сдвига $\tau(g)$ рачионально тогда и только тогда, когда:

а) либо $\operatorname{Fix} \mathscr{J}_{g}^{s}=\mathbb{R}$ и группа $\mathscr{J}_{g}$ ииклическая;

б) либо Fix $\mathscr{J}_{g}^{s} \neq \mathbb{R}$.

Другой способ построения характеров для групп $G \subseteq \mathrm{Homeo+}_{+}(\mathbb{R})$ связан с наличием у таких груп нормальной подгруппы с инвариантной мерой. Для групшы $G \subseteq$ Homeo+ $_{+}(\mathbb{R})$, следуя работе [33], опишем процедуру построения характера по нормальной подгрупе $\Gamma \subseteq G$ с инвариантной мерой $\mu$. Если $g$ - гомеоморфизм, то определим меру $g_{*} \mu$ по следуюшему правилу: для любого борелевского множества $B$ должно выполняться условие $g_{*} \mu(B)=\mu\left(g^{-1}(B)\right)$. Легко видеть, что вся орбита $G_{*}(\mu)=\left\{g_{*} \mu: g \in G\right\}$ состоит из мер, также инвариантных относительно подгруппы $Г$. Если $\tau_{\mu, \Gamma} \neq 0$ в $\operatorname{Hom}(\Gamma, \mathbb{R})$, то по предложению 5.4 для любого $g \in G$ имеет место соотношение $\tau_{g_{*}, \Gamma}=c(g) \tau_{\mu, \Gamma}$, где $c(g)>0$.

Характер $A_{G, \Gamma} \in \operatorname{Hom}(G, \mathbb{R})$ определим следующим образом: для любого $g \in G$ положим

$$
A_{G, \Gamma}(g)=\log c(g) .
$$

Очевидно, при $\Gamma=G$ характер $A_{G, \Gamma}$ тривиальньй, а мера $\mu$ является инвариантной относительно всей групшы $G$. Для таких групп нетривиальньй характер следует строить по первому способу, описанному вьше.

Вместе с тем, сушествуют классы груп, для которых $\Gamma \neq G$ и $A_{G, \Gamma}=0$ в $\operatorname{Hom}(G, \mathbb{R})$. Опишем такие классы груп. 
ПРЕДЛОЖЕНИЕ 5.6 [11]. Пусть $G \subseteq \mathrm{Homeo}_{+}(\mathbb{R}), \quad$ a $\Gamma \subseteq G-$ нормальная подгруппа с инвариантной мерой $\mu$ такой, что $\tau_{\mu, \Gamma} \neq 0$ в $\operatorname{Hom}(\Gamma, \mathbb{R})$. Тогда $A_{G, \Gamma} \neq 0$ в $\operatorname{Hom}(G, \mathbb{R})$ тогда и только тогда, когда $G \neq C_{G}$.

Для полного описания класса груп $G \subseteq \mathrm{Homeo}_{+}(\mathbb{R})$, допускаюших построение характера вторым способом, нам следует исследовать вопрос о сушествовании для них нормальных подгрупп $\Gamma$ с нетривиальньм характером $\tau_{\mu, \Gamma}$.

Лемма 5.1. Пусть $Г \subseteq$ Нотео+ $_{+}(\mathbb{R})$. Тогда следующие условия эквивалентны:

1) для группь Г существует инвариантная борелевская мера $\mu$, конечная на компактах, для которой характер $\tau_{\mu, \Gamma} \neq 0$ в $\operatorname{Hom}(\Gamma, \mathbb{R}) ;$

2) для группы Г существует инвариантная борелевская мера $\mu$, конечная на компактах, и свободно действующий әлемент;

3) фактор-группа $\Gamma /\left\langle\Gamma^{s}\right\rangle$ нетривиальная.

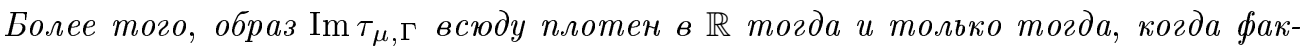
тор-группа $\Gamma /\left\langle\Gamma^{s}\right\rangle$ нечиклическая.

ДокАЗАТЕЛЬСтво. Покажем эквивалентность условий 1) и 3). В силу замечания 5.1 из условия 1) следует условие 3 ). Пусть выполняется условие 3 ). По теореме 5.2 (п. в)) для такой групшы сушествует инвариантная борелевская мера, конечная на компактах, а из замечания 5.1 следует нетривиальность характера $\tau_{\mu, \Gamma}$.

Установим эквивалентность условий 2) и 3 ). Пусть справедливо условие 3 ). Тогда по теореме 5.2 (п. в)) существует инвариантная мера. Так как $\Gamma \neq \Gamma^{s}$, то сушествует свободно действуюший элемент. Пусть справедливо условие 2). Из существования инвариантной меры и теоремы 5.1 следует, что $\mathrm{Fix}^{s} \neq \varnothing$. Следовательно, $\Gamma^{s}$ образует подгруппу. Так как существует свободно действуюший элемент, т.е. $\Gamma \neq \Gamma^{s}$, то фактор-группа $\Gamma / \Gamma^{s}$ нетривиальная.

И, наконец, по тому же замечанию 5.1 образ $\operatorname{Im} \tau_{\mu, \Gamma}$ характера $\tau_{\mu, \Gamma}$ и фактор-группа $\Gamma /\left\langle\Gamma^{s}\right\rangle$ изоморфны, откуда и следует последнее утверждение леммы.

СлЕДСтВИЕ 5.2. Пусть $G \subseteq \mathrm{Homeo}_{+}(\mathbb{R}) u \Gamma \subseteq G$. Если для группь $Г$ существует инвариантная борелевская мера $\mu$, конечная на компактах, для которой характер $\tau_{\mu, \Gamma} \neq 0$ в $\operatorname{Hom}(\Gamma, \mathbb{R})$, то для исходной группь $G$ существует непустое минимальное множество.

ДокАЗАТЕльство. В силу леммы 5.1 справедливо условие $\Gamma \neq \Gamma^{s}$, откуда следует, что $G \neq G^{s}$. Тогда по предложению 3.1 для группы $G$ сушествует непустое минимальное множество.

Итак, групшы $G$, для которых сушествуют нормальные подгруппы $\Gamma$ с нетривиальным характером $\tau_{\mu, \Gamma} \neq 0$, относятся к классу групп с непустьм минимальньм множеством. Дальнейшее изучение этого вопроса будет проведено в $\S 7$ и будет связано с сушествованием 0-максимальных подгрупп.

В конще $\S 3$ была определена каноническая подгруппа $H_{G}$. Выясним, какова ее структура для групп с инвариантной мерой.

Лемма 5.2. Пусть $G \subseteq \operatorname{Homeo(}(X)$. Если для группы $G$ существует инвариантная борелевская мера $\mu$, конечная на компактах, то $H_{G}=G^{s}$. Если при этом минимальное множество недискретное, то $H_{G}=G^{s}=G_{\infty}^{s}$. 
ДокАЗАТЕЛЬСТвО. Из определения нормальной подгруппы $H_{G}$ следует, что $H_{G} \subseteq G_{+}$. Поэтому нам достаточно доказать лемму для групा $G \subseteq \mathrm{Homeo}_{+}(X)$. По предложению 5.3 для таких групп минимальное множество непусто. Если минимальное множество дискретное, то $H_{G}=G^{s}$ по определению. Пусть минимальное множество недискретное. Из существования инвариантной меры следует, что $\operatorname{Fix} G^{s} \neq \varnothing$ (теорема 5.1). Так как минимальное множество принадлежит множеству Fix $G^{s}$ (теорема 3.8 ), то $H_{G}=G^{s}$. По теореме 4.1 фактор-группа $G /\left\langle G^{s}\right\rangle$ нетривиальная. Тогда по лемме $2.3\left\langle G^{s}\right\rangle=G^{s}=G_{\infty}^{s}$.

\section{§6. Проективно-инвариантные меры}

K следуюшему по сложности классу метрических инвариантов относятся проективно-инвариантные меры, являющиеся обобщением инвариантных мер. Вопрос о существовании проективно-инвариантной меры для груп гомеоморфизмов прямой специального типа возникает, в частности, при изучении слоений коразмерности один, порожденных потоками Аносова [33], а также групп квазиконформных преобразований верхней полуплоскости [14].

ОПрЕдЕЛЕниЕ 6.1. Борелевская мера $\mu$, конечная на компактах, называется проективно-инвариантной относительно групшы $G \subseteq \operatorname{Homeo}(\mathbb{R})$, если для любого $g \in G$ сушествует число $c(g)>0$ такое, что для всякого борелевского множества $B \subseteq \mathbb{R}$ вьполняется условие $\mu\left(g^{-1}(B)\right)=c(g) \mu(B)$. Число $c(g)$ называется числом растяжения меры $\mu$ гомеоморфизмом $g \in G$.

Очевидно, что в случае $c(g)=1$ для любого $g \in G$ проективно-инвариантная мера является инвариантной. Для груп $\operatorname{Homeo}\left(S^{1}\right)$ определение проективно-инвариантной меры корректно только лиш в случае $c(g)=1$ для любого $g \in G$. Поэтому для таких груп определение проективно-инвариантной меры переходит в определение инвариантной меры.

В силу теоремы о фактор-группе для группы $G \subseteq \mathrm{Homeo+}_{+}(\mathbb{R})$ с инвариантной мерой фактор-группа исходной группы $G$ по нормальной подгрупе, состоящей из всех стабилизаторов точек на $\mathbb{R}$, изоморфна групе сдвигов. Соответственно, для групшы $G \subseteq \mathrm{Homeo}_{+}(\mathbb{R})$ с проективно-инвариантной мерой фактор-групша исходной группы $G$ по нормальной подгрупше, состоящей из всех стабилизаторов точек на $\mathbb{R}$, для которых рассматриваемая проективно-инвариантная мера является инвариантной, оказывается изоморфной группе аффинных преобразований (теорема 6.1).

Для циклической группы афффинных преобразований $G=\langle g\rangle, g(t)=2 t$, имеется инвариантная мера $\mu$ и проективно-инвариантная мера $\mu_{1}$. Инвариантная мера сосредоточена в точке 0 , являюшейся минимальньм множеством, и мера этой точки равняется 1. Проективно-инвариантная мера сосредоточена в точках $2^{k}, k \in \mathbb{Z}$, и мера каждой точки $2^{k}, k \in \mathbb{Z}$, равна $2^{k}$. Носитель такой проективно-инвариантной меры не является минимальным множеством. В действительности, такие группы следует изучать как групшы с инвариантной мерой.

Поэтому группы с проективно-инвариантной мерой следует изучать в случае, когда для них не существует инвариантной меры. Для таких групп, в отличие от групп с инвариантной мерой, не существует чисто топологического критерия существования 
проективно-инвариантной меры, хотя сушествуют различные критерии, сформулированные в терминах канонически выделенных подгрупп. Критерий, использующий топологические характеристики, содержит условия и алгебраического характера (теорема 6.4). Как и для инвариантной меры, для проективно-инвариантной меры весьма важно описать носитель. Оказьвается, что для изучаемых групп носитель проективно-инвариантной меры также сосредоточен на минимальном множестве (предложение 6.3). Знания о структуре минимального множества позволяют установить эргодические свойства проективно-инвариантной меры (теорема 6.5). Все отмеченные результаты получены для групп гомеоморфизмов $G \subseteq \mathrm{Homeo}_{+}(X)$, сохраняюших ориентацию. Показано, что для произвольной групшы $G \subseteq \operatorname{Homeo}(X)$ сушествование проективно-инвариантной меры эквивалентно существованию проективно-инвариантной меры для максимальной нормальной подгруппы $G_{+}$сохраняюших ориентацию гомеоморфизмов (теорема 6.6).

Далее для групп гомеоморфизмов $G \subseteq \mathrm{Homeo}_{+}(\mathbb{R})$ с проективно-инвариантной мерой $\mu$ каноническим образом будет определен характер $\mathscr{A}_{\mu, G}$, а по нему определены канонические нормальные подгруппы. В случае инвариантности меры $\mu$ характер $\mathscr{A}_{\mu, G}$ тривиальньй. Описьвается структура канонически выделенных подгрупп (леммы 6.1-6.3), а также свойства фактор-групп исходной групш по этим подгруппам (теорема 6.1). Сформулировано два признака существования проективно-инвариантной меры, основанных на метрических свойствах подгрупп исходной групшы (предложения $6.1,6.2$ ). Для груп, не имеюших инвариантной меры, сформулированы критерии сушествования проективно-инвариантной меры, основанные на свойствах канонически выделенных подгрупп (теоремы 6.2-6.4). Дано описание носителя проективно-инвариантной меры (предложение 6.3). Изучены свойства проективно-строгой эргодичности группы $G \subseteq$ Homeо+ $_{+}(\mathbb{R})$ (теорема 6.5). Установлена эквивалентность вопроса сушествования проективно-инвариантной меры для группы $G \subseteq \operatorname{Homeo}(\mathbb{R})$ и ее нормальной подгрупшы $G_{+}$(теорема 6.6), а также связь между их свойствами строгой эргодичности (теорема 6.7). Для групшы $G \subseteq \widetilde{\text { Нотео }_{+}}\left(S^{1}\right)$ сушествование проективно-инвариантной меры эквивалентно существованию инвариантной меры (предложение 6.5), а для групшы $G \subseteq$ Homeо+ $_{+}\left(S^{1}\right)$ всякая проективно-инвариантная мера является инвариантной.

6.1. Характеры и канонические подгруппы, определяемые числом растяжения. Для групп $G \subseteq$ Homeo $_{+}(\mathbb{R})$ с проективно-инвариантной мерой опишем процедуру построения характера. Следуя [32], по борелевской мере $\mu$, конечной на компактах и проективно-инвариантной относительно группы $G \subseteq \mathrm{Homeo}_{+}(\mathbb{R})$, построим характер $\mathscr{A}_{\mu, G}: G \rightarrow \mathbb{R}$ по следующему правилу: для любого $g \in G$ положим $\mathscr{A}_{\mu, G}(g)=\log c(g)$ (см. определение 6.1). Очевидно, что $\mathscr{A}_{\mu, G}=0$ тогда и только тогда, когда проективно-инвариантная мера $\mu$ является инвариантной.

Рассмотрим множества

$$
\mathscr{C}_{\mu, G}=\operatorname{ker} \mathscr{A}_{\mu, G}, \quad \mathscr{H}_{\mu, G}=\operatorname{ker} \mathscr{A}_{\mu, G} \cap G^{s} .
$$

Очевидно, что $\mathscr{C}_{\mu, G}$ - это максимальная нормальная подгруппа групшы $G$, относительно которой мера $\mu$ является инвариантной. Если для нормальной подгруппы $\mathscr{C}_{\mu, G}$ характер $\tau_{\mu, \mathscr{C}_{\mu, G}} \in \operatorname{Hom}\left(\mathscr{C}_{\mu, G}, \mathbb{R}\right)$ нетривиальный, то характеры $\mathscr{A}_{\mu, G}$ и $A_{G, \Gamma}$ совпадают (определение $A_{G, \Gamma}$ в разделе 5.3). 
Для групп с проективно-инвариантной мерой опишем фактор-групшы $\mathscr{C}_{\mu, G} / \mathscr{H}_{\mu, G}$, $G / \mathscr{C}_{\mu, G}, G / \mathscr{H}_{\mu, G}$

Теорема $6.1[10]$. Пусть $G \subseteq$ Homeо $_{+}(\mathbb{R})$ и для $G$ существует проективно-инвариантная борелевская мера, конечная накомпактах. Тогда $\mathscr{H}_{\mu, G} \subseteq \mathscr{C}_{\mu, G} \subseteq G$, $\mathscr{H}_{\mu, G}$ и $\mathscr{C}_{\mu, G}$ - нормальные подгруппь группь $G$, фактор-группа $G / \mathscr{H}_{\mu, G}$ изоморфна группе аффинных преобразований $\mathbb{R}$, а фактор-группьи $\mathscr{C}_{\mu, G} / \mathscr{H}_{\mu, G}, G / \mathscr{C}_{\mu, G}$ изоморфны подгруппам аддитивной группь $\mathbb{R}$. Более того, $\mathscr{C}_{\mu, G}^{s}=\mathscr{H}_{\mu, G}$.

Структура нормальных подгрупп $\mathscr{H}_{\mu, G}$ и $\mathscr{C}_{\mu, G}$ может быть уточнена.

Лемма 6.1 [10]. Пусть $G \subseteq$ Homeо $_{+}(\mathbb{R})$ и для $G$ существует проективно-инвариантная борелевская мера, конечная на компактах, но не существует инвариантной мерь. Тогда $G \neq \mathscr{C}_{\mu, G}, \mathscr{C}_{\mu, G} \neq \mathscr{H}_{\mu, G}$, а фактор-группа $\mathscr{C}_{\mu, G} / \mathscr{H}_{\mu, G}$ нечиклическая.

ЛЕмма 6.2 [10]. Пусть $G \subseteq$ Homeо+ $_{+}(\mathbb{R})$ и для $G$ существует проективно-инвариантная борелевская мера, конечная на компактах. Если $\mathscr{C}_{\mu, G} \neq \mathscr{H}_{\mu, G}$, то $\mathscr{H}_{\mu, G}=G_{\infty}^{s}$. Более того, $G \backslash G^{s} \neq \varnothing u G \backslash G^{s} \subseteq \mathscr{C}_{\mu, G}$.

Теперь мы можем полностью описать структуру нормальных подгрупп $\mathscr{H}_{\mu, G}$ и $\mathscr{C}_{\mu, G}$.

Лемма 6.3. Пусть $G \subseteq$ Нотео $_{+}(\mathbb{R})$ и для $G$ существует проективно-инвариантная борелевская мера $\mu$, конечная на компактах, но не существует инвариантной меры. Тогда $\mathscr{H}_{\mu, G}=H_{G}$ и $\mathscr{C}_{\mu, G}=C_{G}$.

ДокАЗАТЕльство. По лемме $6.2 G \backslash G^{s} \neq \varnothing$, т.е. существует свободно действующий элемент $\widetilde{g} \in \mathscr{C}_{\mu}, G$. Тогда по лемме 3.1 для группы $G$ сушествует минимальное множество. Так как элемент $\widetilde{g}$ свободно действуюший, то минимальное множество не ограничено ни слева, ни справа. Из условия нецикличности фактор-группы $\mathscr{C}_{\mu, G} / \mathscr{H}_{\mu, G}$ и того, что $\mathscr{C}_{\mu, G}^{s}=\mathscr{H}_{\mu, G}$ (теорема 6.1 ), следует, что минимальное множество группы $G$ (мы обозначаем его через $E(G)$ ) недискретное. Из недискретности минимального множества $E(G)$, его неограниченности слева и справа и условия $\mathscr{H}_{\mu, G}=G_{\infty}^{s}$ (см. лемму 6.2) следует, что $H_{G} \subseteq \mathscr{H}_{\mu, G}$.

$\mathrm{C}$ другой стороны, так как $\widetilde{g} \in \mathscr{C}_{\mu, G}-$ свободно действуюший элемент, то в силу леммы 3.1 минимальное множество группы $\mathscr{C}$ также непусто. Из нецикличности фактор-групшы $\mathscr{C}_{\mu, G} / \mathscr{H}_{\mu, G}$ и условия $\mathscr{C}_{\mu, G}^{s}=\mathscr{H}_{\mu, G}$ (теорема 6.1) следует, что такое минимальное множество недискретное (мы обозначаем его через $E\left(\mathscr{C}_{\mu, G}\right)$ ). Тогда по лемме $3.4 E(G)=E\left(\mathscr{C}_{\mu, G}\right)$. В силу определения подгруппа $\mathscr{C}_{\mu, G}$ является максимальной нормальной подгруппой, для которой проективно-инвариантная мера $\mu$ является инвариантной. Тогда по теореме 5.1 должно выполняться условие $\mathrm{Fix} \mathscr{C}_{\mu, G}^{s} \neq \varnothing$. Очевидно, что минимальное множество $E\left(\mathscr{C}_{\mu}, G\right)$ должно принадлежать замкнутому множеству Fix $\mathscr{C}_{\mu, G}^{s}$, инвариантному относительно $\mathscr{C}_{\mu, G}$. В таком случае должно выполняться включение $\mathscr{C}_{\mu, G}^{s} \subseteq H_{G}$. Так как $\mathscr{C}_{\mu, G}^{s}=\mathscr{H}_{\mu, G}$, то это влечет за собой включение $\mathscr{H}_{\mu, G} \subseteq H_{G}$. Сравнивая с полученньм ранее обратньм включением, имеем, что $H_{G}=\mathscr{H}_{\mu, G}$.

Из условий $G \backslash G^{s} \subseteq \mathscr{C}_{\mu, G}$ и $\mathscr{H}_{\mu, G}=G_{\infty}^{s}$ (лемма 6.2) следует включение $C_{G} \subseteq$ $\mathscr{C}_{\mu, G}$. Так как мера $\mu$ инвариантна относительно группы $\mathscr{C}_{\mu, G}$ и сушествует свободно 
действующий элемент $\widetilde{g} \in \mathscr{C}_{\mu}, G$, то верно и обратное включение $\mathscr{C}_{\mu, G} \subseteq C_{G}$. Лемма доказана.

6.2. Топологические и алгебраические критерии существования проективно-инвариантной меры. Сформулируем два достаточных условия сушествования проективно-инвариантной меры.

ПРЕДЛОЖЕНИЕ 6.1. Пусть $G \subseteq \mathrm{Homeo}_{+}(\mathbb{R})$, a $\Gamma$ - нормальная подгруппа. Ecли фактор-группа $\Gamma /\left\langle\Gamma^{s}\right\rangle$ нечиклическая, то для исходной группь $G$ существует проективно-инвариантная борелевская мера, конечная на компактах.

Результат, эквивалентный предложению 6.1, был доказан в работе [33]. Там условия формулировались в терминах характера $\tau_{\mu, \Gamma} \in \operatorname{Hom}(\Gamma, \mathbb{R})$. Такую переформулировку легко восстановить с помощью леммы 5.1 .

ПРЕДЛОЖЕНИЕ 6.2 [33]. Пусть $G \subseteq$ Homeо+ $_{+}(\mathbb{R})$, a $Г$ - нормальная подгруппа с инвариантной борелевской мерой $\mu$, конечной на компактах. Если $\tau_{\mu, \Gamma \neq 0}$ в $\operatorname{Hom}(\Gamma, \mathbb{R})$, а $A_{G, \Gamma} \neq 0$ в $\operatorname{Hom}(G, \mathbb{R})$, то мера $\mu$ является проективно-инвариантной относительно исходной группь $G$.

Для групп, не имеющих инвариантной меры, мы можем сформулировать критерии существования проективно-инвариантной меры, используюшие свойства канонически выделенных подмножеств исходной групшы.

Tеорема 6.2 [11]. Пусть $G \subseteq$ Homeo+ $_{+}(\mathbb{R})$ и для $G$ не существует инвариантной борелевской мерь, конечной на компактах. Для существования борелевской меры, конечной на компактах и проективно-инвариантной относительно группь $G$, необходимо и достаточно, чтобь подмнохсества $G_{\infty}^{s}$ и $C_{G}$ являлись подгруппами и выполнялось условие $C_{G} \neq G_{\infty}^{s}$.

Ниже будет описан критерий сушествования проективно-инвариантной меры, используюший свойства нормальных подгрупп и непосредственно следующий из теоремы 6.1 и предложения 6.1 .

Теорема 6.3. Пусть $G \subseteq$ Hотео+ $_{+}(\mathbb{R})$ и для $G$ не существует инвариантной борелевской мерь, конечной на компактах. Для существования борелевской меры, конечной на компактах и проективно-инвариантной относительно группь $G$, необходимо и достаточно, чтобь существовала нормальная подгруппа $\Gamma \subseteq G$ со свойствами: $\left\langle\Gamma^{s}\right\rangle=\Gamma^{s}$, а фактор-группа $\Gamma / \Gamma^{s}$ нечиклическая.

ДокаЗАТЕльство. Необходимость. По теореме $6.1 \mathscr{C}_{\mu, G}^{s}=\mathscr{H}_{\mu, G}$, а по лемме 6.1 фактор-группа $\mathscr{C}_{\mu, G} / \mathscr{H}_{\mu, G}$ не циклическая. Остается положить $\Gamma$ равной $\mathscr{C}_{\mu}, G$.

Достаточность следует из предложения 6.1.

Сформулируем важный критерий существования проективно-инвариантной меры, использующий как топологические, так и алгебраические характеристики. Для любого элемента $q \in \mathrm{Homeo+}_{+}(\mathbb{R})$ будем пользоваться обозначениями:

$$
\begin{aligned}
& T_{q}=\sup \{t: q(t)=t\}, \quad t_{q}=\inf \{t: q(t)=t\}, \quad \text { если } \operatorname{Fix}\langle q\rangle \neq \varnothing ; \\
& T_{q}=t_{q}=-\infty, \quad \text { если } \operatorname{Fix}\langle q\rangle=\varnothing .
\end{aligned}
$$


Tеорема 6.4 [13]. Пусть $G \subseteq$ Homeo+ $_{+}(\mathbb{R})$, для которой не существует борелевской инвариантной мерь, конечной на компактах. Для существования борелевской меры, конечной на компактах и проективно-инвариантной относительно группы $G$, необходимо и достаточно, чтобы одновременно выполнялись условия:

1) множество $G_{\infty}^{s}$ является подгруппой и фактор-группа $G / G_{\infty}^{s}$ некоммутативная;

2) для всякого $g \in G^{s} \backslash C_{G}$ справедливы условия: $t_{g}, T_{g}$ конечны и для любых $t \in]-\infty, t_{g}[, T \in] T_{g},+\infty[$ выполнено соотношение $\operatorname{sign}[g(t)-t]=$ $-\operatorname{sign}[g(T)-T]$

3) для любьх $g_{1}, g_{2} \in G^{s} \backslash C_{G}$ либо $t_{g_{1}}=t_{g_{2}} u T_{g_{1}}=T_{g_{2}}$, либо $\left[t_{g_{1}}, T_{g_{1}}\right] \cap$ $\left[t_{g_{2}}, T_{g_{2}}\right]=\varnothing$.

6.3. Эргодические свойства проективно-инвариантной меры. Опишем носители проективно-инвариантных мер. Носители инвариантных мер мы описали в разделе 5.2. Поэтому важно рассмотреть групшы с проективно-инвариантной мерой, для которых не сушествует инвариантной меры.

ПрЕДЛОЖЕНИЕ 6.3. Пусть $G \subseteq$ Homeo+ $_{+}(\mathbb{R})$ и для $G$ существует проективно-инвариантная борелевская мера $\mu$, конечная на компактах, но не существует инвариантной меры. Тогда справедливы следующие утверэсдения:

1) для группь $G$ существует единственное недискретное минимальное множсество $E(G)$;

2) $C_{G}$ является нормальной подгруппой, а минимальные множества группьи $G$ и нормальной подгруппь $C_{G}$ совпадают;

3) мера н инвариантна относительно подгруппь $C_{G}$, а ее носитель совпадает с минимальным множеством $E(G)$ группь $G$.

ДокАЗАТЕльство. По лемме $6.3 C_{G}$ совпадает с $\mathscr{C}_{\mu, G}$ и поэтому является нормальной подгруппой. Так как $\mathscr{H}_{\mu, G}=G_{\infty}^{s}\left(\right.$ лемма 6.2), $\mathscr{C}_{\mu, G}^{s}=\mathscr{H}_{\mu, G}$ (теорема 6.1), то в силу теоремы 4.1 для группы $C$ существует единственное недискретное минимальное множество. Тогда пш. 1) и 2) будут следовать из леммы 3.4 .

В силу определения $\mathscr{C}_{\mu}, G$ является максимальной нормальной подгруппой, для которой мера $\mu$ является инвариантной. Так как $\mathscr{C}_{\mu, G}=C_{G}$, а $E\left(C_{G}\right)=E(G)$ - единственное недискретное минимальное множество группы $C_{G}$, то по теореме 5.8 supp $\mu=$ $E\left(C_{G}\right)$.

ЗАмЕчАнИЕ 6.1. Из предложения 6.2 следует, что для группы $G \subseteq \mathrm{Homeo}_{+}(\mathbb{R})$, не имеюшей инвариантной меры, все свойства проективно-инвариантной меры $\mu$ могут быть описаны как свойства меры, инвариантной относительно нормальной подгрупшы $C_{G} \subseteq G$.

Помимо предложения 6.3 сформулируем одно простое, но важное утверждение о сушествовании минимальных множеств.

ПреДЛОЖЕНИЕ 6.4. Пусть $G \subseteq \operatorname{Homeo}(\mathbb{R})$ и для $G$ существует проективно-инвариантная борелевская мера, конечная на компактах. Тогда существует непустое минимальное мнохсество. 
ДокАЗАТЕльство. В силу теоремы 3.9 достаточно рассматривать групшы $G \subseteq$ $\mathrm{Homeo}_{+}(\mathbb{R})$. Если для группы $G$ сушествует инвариантная мера, то доказательство следует из предложения 5.2. Пусть для групшы $G$ не существует инвариантной меры. В силу лемм 6.1 и 6.2 справедливо условие $G \backslash G^{s} \neq \varnothing$. Тогда по теореме 3.3 (п. в)) для группы $G$ сушествует непустое минимальное множество.

Детальное описание носителей проективно-инвариантных мер во всех остальных случаях проведено в [10]. Для групп $G \subseteq \mathrm{Homeo+}_{+}(\mathbb{R})$ с инвариантной мерой проективно-инвариантная мера может иметь носитель и вне минимального множества группы $G$.

ОПРЕДЕлЕНИЕ 6.2. Група $G \subseteq \operatorname{Homeo}(\mathbb{R})$ назьвается проективно-строго әрго-

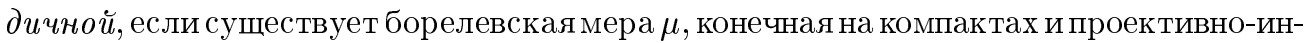
вариантная относительно группы $G$, и для любой борелевской меры $\mu_{1}$, конечной на компактах и проективно-инвариантной относительно группы $G$, справедливо условие $\mu_{1}=\lambda \mu, \lambda \in \mathbb{R}^{+}$.

Теорема 6.5. Пусть $G \subseteq$ Homeо+ $_{+}(\mathbb{R})$ и для $G$ существует борелевская мера $\mu$, конечная на компактах и проективно-инвариантная относительно груп$n$ пь $G$, но не существует инвариантной борелевской меры, конечной на компакmax. Тогда группа $G$ будет проективно-строго әргодичной.

ДокАЗАТЕльство. Пусть $\mu_{1}$ - некоторая другая проективно-инвариантная мера. По предложению 6.2 меры $\mu$ и $\mu_{1}$ инвариантны относительно подгруппы $C_{G}$. Так как минимальное множество группы $C_{G}$ недискретное, то по теореме 5.9 группа $C_{G}$ строго эргодичная, т.е. $\mu_{1}=\lambda \mu, \lambda \in \mathbb{R}^{+}$, что и завершает доказательство теоремы.

Теперь мы в состоянии описать связь между проективно-инвариантньми мерами групшы $G \subseteq \operatorname{Homeo}(\mathbb{R})$ и ее нормальной подгрупшы $G_{+}$.

Теорема 6.6. Пусть $G \subseteq \operatorname{Homeo}(\mathbb{R})$. Для группь $G$ проективно-инвариантная мера существует тогда и только тогда, когда существует проективно-инвариантная мера для нормальной подгруппьи $G_{+}$.

ДоКАЗАТЕЛЬСТВо. В одну сторону доказательство очевидно. Пусть для нормальной подгрупшы $G_{+}$сушествует проективно-инвариантная мера $\mu$. Если для $G_{+}$сушествует инвариантная мера, то по теореме 5.10 для исходной группы $G$ также существует инвариантная мера, что и завершает доказательство. Остается рассмотреть случай, когда для $G_{+}$не сушествует инвариантной меры. Очевидно, что следует рассматривать случай $G \neq G_{+}$. Мы знаем, что $G=G_{+} \cup f G_{+}$, где $f$ - некоторьй элемент из $G \backslash G_{+}$. Если $q$ - гомеоморфизм, то определим меру $q_{*} \mu$ по следующему правилу: для любого борелевского множества $B$ должно вьполняться условие $q_{*} \mu(B)=\mu\left(q^{-1}(B)\right)$. Из нормальности подгруппы $G_{+}$следует, что для любого элемента $g \in G$ мера $g_{*} \mu$ также проективно-инвариантна относительно подгрупшы $G_{+}$. Тогда по теореме 6.5 для любого $g \in G$ справедливы соотношения $g_{*} \mu=d_{g} \mu, d_{g}>0$, т.е. мера $\mu$ является проективно-инвариантной относительно исходной группы $G$.

Опишем связь между свойствами строгой эргодичности группы $G \subseteq \operatorname{Homeo}(\mathbb{R})$ и нормальной подгруппы $G_{+}$. 
Теорема 6.7. Пусть $G \subseteq$ Нотео $(\mathbb{R})$ и существует борелевская мера, конечная на компактах и проективно-инвариантная относительно группь $G$. Если подгруппа $G_{+}$проективно-строго әргодичная, то и группа $G$ будет проективно-строго әргодичной. Если для подгруппь $G_{+}$не существует инвариантной меры, то группа $G$ и подгруппа $G_{+}$проективно-строго әргодичны.

ДокаЗАТЕльство. Первое утверждение очевидно. Докажем второе утверждение. Проективно-строгая эргодичность подгрупшы $G_{+}$следует из теоремы 6.5. Пусть $\mu_{1}, \mu_{2}$ - две проективно-инвариантные меры относительно группы $G$. Очевидно, что $\mu_{1}, \mu_{2}$ являются проективно-инвариантньми мерами и относительно подгруппы $G_{+}$. Тогда из проективно-строгой эргодичности подгрупшы $G_{+}$следует условие $\mu_{2}=\lambda \mu_{1}$, $\lambda>0$, что и доказывает проективно-строгую эргодичность исходной группы $G$.

И последнее. Какими дополнительными свойствами обладают группы $G \subseteq$ $\widehat{\mathrm{HomeO}_{+}}\left(S^{1}\right)$ с проективно-инвариантной мерой?

ПРЕДЛОЖЕНИЕ 6.5 [10]. Пусть $G \subseteq \widetilde{\text { Homeo }_{+}}\left(S^{1}\right)$. Для групnь $G$ проективно-инвариантная мера существует тогда и только тогда, когда существует инвариантная мера.

\section{$\S$ 7. $\omega$-проективно-инвариантные меры}

Одним из первых результатов по метрическим инвариантам является теорема Боголюбова-Крылова о сушествовании инвариантной меры для гомеоморфизма окружности. Мы уже отмечали, что при исследовании групп гомеоморфизмов прямой центральным является описание различных метрических инвариантов. Такой раздел исследований известен как теория Боголюбова-Крылова, и теоремы сушествования метрических инвариантов естественно называть теоремами Боголюбова-Крылова. Перейдем к описанию некоторого более общего метрического инварианта, обобщающего понятие проективно-инвариантной меры.

Пусть $\mathscr{M}$ обозначает пространство борелевских мер на $X$, конечных на компактах ( $\mathscr{M}^{+}$- конус положительных мер). В случае $X=\mathbb{R}$ пространство $\mathscr{M}$ рассматривается как сопряженное пространство к пространству $\mathscr{R}(\mathbb{R})$ непрерывных функций на $\mathbb{R}$ с компактньм носителем и топологией индуктивного предела [53; гл. I, дополнение]. В случае $X=S^{1}$ пространство $\mathscr{M}$ рассматривается как сопряженное пространство к пространству $C\left(S^{1}\right)$ непрерьвных функций на $S^{1}$. Для группы $G \subseteq \operatorname{Homeo}(X)$ через $G_{*}$ будем обозначать изоморфную ей группу непрерьвных линейных операторов, действуюших на пространстве $\mathscr{M}$. Изоморфизм $\theta: G \rightarrow G_{*}$, где $\theta(g)=g_{*}$, определяется следуюшим образом: для любой меры $\mu$ и любого борелевского множества $B$ $g_{*} \mu(B)=\mu\left(g^{-1}(B)\right)$.

Заметим, что конус положительных мер $\mathscr{M}^{+}$инвариантен относительно группы непрерьвных линейных операторов $G_{*}$. Для любой меры $\mu \in \mathscr{M}^{+}$через $\mathscr{K}_{\mu}(G)$ обозначим замкнутьй выпукльй конус, порожденньй орбитой $G_{*}(\mu)=\left\{g_{*} \mu\right\}_{g \in G}$ меры $\mu$. Очевидно, что конус $\mathscr{K}_{\mu}(G)$ инвариантен относительно группы непрерьвных линейных операторов $G_{*}$.

ОПРЕДЕЛЕНИЕ 7.1. Пусть $G \subseteq \operatorname{Homeo}(X)$, а $\mu \in \mathscr{M}^{+}$. Конус $\mathscr{K}_{\mu}(G)$ назьвается минимальнылм, если для любой меры $\bar{\mu} \in \mathscr{K}_{\mu}(G)$ справедливо условие $\mathscr{K}_{\mu}(G)=$ $\mathscr{K}_{\bar{\mu}}(G)$. 
Пусть $\mathscr{K} \subseteq \mathscr{M}^{+}-$конус и $\mu \in \mathscr{K}$. Луч $\lambda \mu, \lambda>0$, называется крайним, если не существует мер $\mu_{1}, \mu_{2} \in \mathscr{K} \backslash \mu$ и неотрицательных чисел $\lambda_{1}, \lambda_{2}$ таких, что $\mu=\lambda_{1} \mu_{1}+\lambda_{2} \mu_{2}$.

ОПРЕДЕЛЕниЕ 7.2. Борелевская мера $\mu \in \mathscr{M}^{+}$, конечная на компактах, назьвается $\omega$-проективно-инвариантной относительно групшы $G \subseteq \operatorname{Homeo}(X)$, если выпукльй конус $\mathscr{K}_{\mu}(G)$ является минимальным, луч $\lambda \mu$ крайним, а $\omega$ является мошностью множества крайних лучей.

В случае $\omega=1$ инвариантный конус $\mathscr{K}_{\mu}(G)$ одномерньй и определение 1 -проективно-инвариантной меры совпадает с определением 6.1 проективно-инвариантной меры. Если инвариантный конус $\mathscr{K}_{\mu}(G)$ не только одномерньй, но и неподвижный относительно групшы линейных операторов $G_{*}$, то проективно-инвариантная мера является инвариантной. По определению инвариантную меру будем назьвать 0-проективно-инвариантной. В дальнейшем, в зависимости от контекста, будем пользоваться эквивалентными названиями: 1-проективно-инвариантная мера - проективно-инвариантная мера; 0-проективно-инвариантная мера - инвариантная мера.

Здесь, как и в предыдущих разделах, важно без априорных предположений о характере группы сформулировать различные критерии сушествования $\omega$-проективно-инвариантной меры. В предыдущих разделах мы отметили, что из сушествования инвариантной меры или проективно-инвариантной меры следует существование непустого минимального множества. Поэтому в дальнейшем при изучении других метрических инвариантов условие сушествования непустого минимального множества будет обязательным. Для группы $G \subseteq \mathrm{Homeo}_{+}(\mathbb{R})$ с непустым минимальным множеством установлен центральньй результат о существовании $\omega$-проективно-инвариантной меры (теорема Боголюбова-Крылова). Показано, что в случае отсутствия проективно-инвариантной меры кардинальное число $\omega$ не может быть конечным (теорема 7.11). Весьма важен вопрос о том, когда для группы с непустым минимальньм множеством, для которой по теореме Боголюбова-Крылова сушествует $\omega$-проективно-инвариантная мера, будет существовать инвариантная либо проективно-инвариантная мера? Этот вопрос связан с изучением максимальных нормальных подгрупп с инвариантной либо проективно-инвариантной мерой. Описана как структура таких максимальных нормальных подгрупп (теоремы 7.1, 7.4), так и структура фактор-групшы исходной групшы по этим подгруппам (теорема 7.5). Оказьвается, что максимальные нормальные подгруппы с инвариантной мерой также обладают экстремальными свойствами: или являются "почти” циклическими, или имеют сложность исходной групшы.

Далее для групп $G \subseteq$ Ноmео+ $_{+}(X)$ с непустым минимальным множеством изучается центральньй вопрос о сушествовании максимальных подгрупп с инвариантной (проективно-инвариантной) мерой. Доказаны сушествование и единственность таких максимальных подгрупп, описана их структура (теоремы 7.1-7.4), а также приводятся критерии их сложности (предложения 7.1, 7.2). Изучен важньй вопрос о структуре фактор-групшы по отмеченным максимальным подгруппам (теоремы 7.5-7.7). Для исследуемых груп $G \subseteq$ Нотео+ $(X)$ сформулирована обобщенная теорема Боголюбова-Крылова о существовании $\omega$-проективно-инвариантной меры (теоремы 7.8-7.10). Изучено множество возможных значений кардинального числа $\omega$ (теоремы $7.11,7.12$ ). 
7.1. Топологический признак существования 0-максимальных и 1-максимальных подгрупп. Структура таких подгрупп.

ОПРЕДЕЛЕНИЕ 7.3. Пусть $G \subseteq$ Нотео $_{+}(X)$. Нормальная подгрупа $M_{0, G} \subseteq G$ $\left(M_{1, G} \subseteq G\right)$ называется 0-максимальной (1-максимальной), если существует борелевская мера, конечная на компактах и инвариантная (проективно-инвариантная) относительно подгруппы $M_{0, G}\left(M_{1, G}\right)$, и она не является собственной подгруппой какой-либо нормальной подгрупы групы $G$, для которой также существует борелевская инвариантная (проективно-инвариантная) мера, конечная на компактах.

В определении перед словом "максимальная" стоит 0 и 1 , ибо инвариантные меры это те же 0-проективно-инвариантные меры, а проективно-инвариантные меры - это те же 1-проективно-инвариантные меры, о которых мы говорили выше.

Teopema 7.1 [11]. Пусть $G \subseteq$ Hотео $_{+}(\mathbb{R})$ и для группь $G$ существует непустое минимальное множество. Тогда для группы $G$ существует, причем единственная, 0-максимальная подгруппа $M_{0, G}$, при этом она

1) совпадает с әруппой $G$, если для группь $G$ существует инвариантная мера;

2) совпадает с нормальной подгруппой $C_{G}$, если для группь $G$ не существует инвариантной мерьи, но существует проективно-инвариантная ме$p a$;

3) содержит нормальную подгруппу $H_{G}$, если для группь $G$ не существует проективно-инвариантной меры и $G=C_{G}$; более того, $M_{0, G}^{s}=H_{G}$ и фактор-группа $M_{0, G} / H_{G}$ - либо тривиальная, либо бесконечная ииклическая группа;

4) совпадает с нормальной подгруппой $H_{G}$, если для группьи $G$ не существует проективно-инвариантной меры и $C_{G} \neq G$.

Более того, любая нормальная подгруппа с инвариантной мерой содержится в 0-максимальной подгруппе.

ДокАЗАТЕЛЬСтво. Существование 0-максимальной подгрупшы со свойствами 1)-4) было доказано в работе [11]. Нам следует доказать единственность 0-максимальной подгруппы и последний пункт теоремы.

1. Если для групшы $G$ сушествует инвариантная мера, то доказательство очевидно.

2. Пусть для группы $G$ существует проективно-инвариантная мера, но не существует инвариантной меры. По предложению 6.4 для группы $G$ существует непустое минимальное множество. Так как не существует инвариантной меры, то $\operatorname{Fix} G^{s}=\varnothing$ (теорема 5.1). Тогда по теореме 3.1 для групшы $G$ минимальное множество $E(G)$ недискретное и, соответственно, единственное.

Предположим, что $Q \subseteq G$ - нормальная подгруппа с инвариантной мерой. По п. 2) теоремы $7.1 C_{G}$ является 0-максимальной подгруппой. Поэтому для доказательства нам достаточно показать справедливость включения $Q \subseteq C_{G}$. По теореме 5.1 множество Fix $Q^{s}$ непусто. Напомним, что $\operatorname{Fix} Q^{s}$ - замкнутое множество. Из нормальности подгруппы $Q$ следует, что множество Fix $Q^{s}$ инвариантно относительно исходной группы $G$. Тогда по теореме $3.1 E(G) \subseteq \mathrm{Fix} Q^{s}$, откуда и будет следовать включение $Q^{s} \subseteq H_{G}$. Заметим, что для группы $Q$ множество $Q^{s}$ образует группу, т.е. 
$Q^{s}=\left\langle Q^{s}\right\rangle$. В случае тривиальной фактор-группы $Q / Q^{s}$ имеют место включения $Q=Q^{s} \subseteq H_{G} \subseteq M_{0, G}=C_{G}$ и доказательство завершено.

Пусть фактор-группа $Q / Q^{s}$ нетривиальная. Тогда по лемме $2.3 Q^{s}=Q_{\infty}^{s}$ откуда будет следовать, что $Q=C_{G}$. Но в таком случае справедливо включение $Q \subseteq C_{G}$, что и завершает доказательство.

3. Пусть для групш $G$ не сушествует проективно-инвариантной меры. В силу пाг. 3) и 4) теоремы 7.1 для группы $G$ сушествует 0-максимальная подгруппа $M_{0, G}$ со свойством $M_{0, G}^{s}=H_{G}$. Предположим, что $Q \subseteq G$ - нормальная подгруппа с инвариантной мерой.

На первом шаге покажем, что подгруппа $Q$ содержится в более широкой нормальной подг руппе $\mathscr{F}$ с инвариантной мерой, для которой справедливо условие $\mathscr{F}^{s}=H_{G}$. Дословно повторяя рассуждения предыдущего второго пункта, получим, что минимальное множество $E(G)$ группы $G$ недискретное и, соответственно, единственное, а для групшы $Q$ справедливы условия: $Q^{s}=\left\langle Q^{s}\right\rangle, E(G) \subseteq \operatorname{Fix} Q^{s}, Q^{s} \subseteq H_{G}$. Если $Q=Q^{s}$, то $Q \subseteq H_{G}=M_{0, G}^{s}$. Следовательно, группа $Q$ содержится в 0-максимальной подгрупе $M_{0, G}$. Если $Q \neq Q^{s}$, то по лемме $2.3 Q=C_{Q}$. Образуем групу $\mathscr{F}=\left\langle Q, H_{G}\right\rangle$. Очевидно, что $H_{G} \subseteq \mathscr{F}^{s}$. Так как минимальное множество $E(G)$ инвариантно относительно групшы $G$, а тем более относительно подгруппы $\mathscr{F}$, то для любого элемента $f \in \mathscr{F}$ справедлива альтернатива: либо $f(t)=t$ для любого $t \in E(G)$, либо $f(t) \neq t$ для любого $t \in E(G)$. Отсюда следует обратное включение $\mathscr{F}^{s} \subseteq H_{G}$ и, соответственно, равенство $\mathscr{F}^{s}=H_{G}$. Так как $H_{G}$ является подгрупшой и $\mathscr{F} \neq \mathscr{F}^{s}$, то по теореме 5.2 для группы $\mathscr{F}$ также существует инвариантная мера.

На втором шаге покажем, что для любой нормальной подгрупшы $\Gamma \subseteq G$ с инвариантной мерой и включением $H_{G} \subseteq \Gamma$ справедливо условие $\Gamma^{s}=H_{G}$. Действительно, для подгрупш $\Gamma$ справедливо включение $H_{G} \subseteq \Gamma^{s}$. По теореме 5.1 множество Fix $\Gamma^{s}$ непусто. Из нормальности подгрупшы $\Gamma$ следует, что множество Fix $\Gamma^{s}$ инвариантно относительно исходной группы $G$. Тогда по теореме $3.1 E(G) \subseteq \mathrm{Fix}^{s} \Gamma^{s}$, откуда и будет следовать обратное включение $\Gamma^{s} \subseteq H_{G}$. Следовательно, $\Gamma^{s}=H_{G}$.

На третьем шаге покажем, что любая нормальная подгруппа $Q \subseteq G$ с инвариантной мерой содержится в некоторой 0-максимальной подгрупее $\widetilde{\Gamma}$ со свойством $\widetilde{\Gamma}^{s}=$ $H_{G}$. Если $Q=Q^{s}$, то на первом шаге мы показали, что $Q$ содержится в 0-максимальной подгруппе $M_{0, G}$. Пусть $Q \neq Q^{s}$. Из первого шага известно, что $Q$ содержится в нормальной подгруппе $\mathscr{F}$ с инвариантной мерой и свойством $\mathscr{F} s=H_{G}$. Доказательство будет завершено, если мы покажем, что подгруппа $\mathscr{F}$ содержится в некоторой 0-максимальной подгрупе. Для этого на множестве всех нормальных подгрупп $\Gamma$ групшы $G$, для которых существуют инвариантные меры и справедливо включение $\mathscr{F} \subseteq \Gamma$, введем отношение частичного порядка следуюшим образом: $\Gamma_{1} \preceq \Gamma_{2}$, если $\Gamma_{1} \subseteq \Gamma_{2}$. Из результата второго шага следует, что для таких групп $Г$ выполняется условие $\Gamma^{s}=H_{G}$. Так как для исходной группы $G$ не сушествует проективно-инвариантной меры, то по предложению 6.1 фактор-групша $\Gamma / H_{G}$ будет циклической. По лемме Цорна цеп $\mathscr{F} \subseteq \mathscr{F}$ содержится в некоторой максимальной цепи $\left\{\Gamma_{\alpha}\right\}_{\alpha \in A}$, причем для любого $\alpha \in A$ фактор-группа $\Gamma_{\alpha} / H_{G}$ циклическая. Рассмотрим объединение $\widehat{\Gamma}=\bigcup_{\alpha \in A} \Gamma_{\alpha}$, задающее нормальную подгруппу группы $G$. Очевидно, что $\widetilde{\Gamma}^{s}=H_{G}$ и $\widetilde{\Gamma} \neq \widetilde{\Gamma}^{s}$. Так как $H_{G}$ - подгруппа, то по теореме 5.2 для $\widetilde{\Gamma}$ существует инвариантная мера. В таком случае $\widetilde{\Gamma}$ будет 0-максимальной подгруппой. Более того, так как для исходной групшы $G$ не существует проективно-инвариантной меры, то по пред- 
ложению 6.1 фактор-группа $\widetilde{\Gamma} / H_{G}$ будет циклической. Заметим, что из утверждений шагов 1 и 2 следует, что любая 0-максимальная подгруппа $\widetilde{\Gamma} \subseteq G$ удовлетворяет условию $\widetilde{\Gamma}^{s}=H_{G}$.

На четвертом шаге покажем, что 0-максимальная подгруппа единственная. Здесь рассмотрим два случая.

Случай первьй, когда $G \neq C_{G}$. В конце третьего шага мы отметили, что для любой 0-максимальной подгрупшы $\widetilde{\Gamma} \subseteq G$ справедливо условие $\widetilde{\Gamma}^{s}=H_{G}$. По п. 4) теоремы 7.1 для групшы $G$, удовлетворяющей условию $G \neq C_{G}$, сушествует 0-максимальная подгруппа $M_{0, G}$, и она совпадает с $H_{G}$. Следовательно, 0-максимальная подгруппа единственна и равна $H_{G}$.

Случай второй, когда $G=C_{G}$. По результатам третьего шага для любой 0-максимальной подгрупшы $\widetilde{\Gamma} \subseteq G$ справедливы условия: $\widetilde{\Gamma}^{s}=H_{G}$; фактор-группа $\widetilde{\Gamma} / H_{G}$ циклическая. Пусть $\widetilde{\Gamma}_{1}, \widetilde{\Gamma}_{2}$ - две несовпадающие 0-максимальные подгрупшы. В таком случае фактор-группы $\widetilde{\Gamma}_{1} / H_{G}, \widetilde{\Gamma}_{2} / H_{G}$ будут нетривиальные. Пусть левьй смежньй класс $\gamma_{1} H_{G}$ определяет образуюшую фактор-грушы $\widetilde{\Gamma}_{1} / H_{G}$, а левый смежньй класс $\gamma_{2} H_{G}$ определяет образующую фактор-групш $\widetilde{\Gamma}_{2} / H_{G}$. Образуем групп $\mathscr{J}=$ $\left\langle\widetilde{\Gamma}_{1}, \widetilde{\Gamma}_{2}\right\rangle$. Так как минимальное множество $E(G)$ будет инвариантным и относительно подгрупшы $\mathscr{J} \subseteq G$, то имеет место изоморфизм $\mathscr{J} / H_{G} \simeq\left\langle\widetilde{\Gamma}_{1} / H_{G}, \widetilde{\Gamma}_{2} / H_{G}\right\rangle$.

Далее мы воспроизведем конструкцию из работы [9]. Пусть $\mu$ является инвариантной мерой для 0-максимальной подгрупшы $\widetilde{\Gamma}_{1}$. Не нарушая обшности, мы можем считать, что $\gamma_{1}(t)>t$ для любого $t \in \mathbb{R}$. Так как $\widetilde{\Gamma}_{1} / H_{G}-$ циклическая, то мы можем так переопределить меру $\mu$, чтобы она удовлетворяла условиям: $\mu$ является инвариантной относительно $\widetilde{\Gamma}_{1}$; мера $\mu$ непрерьвна и ее носитель совпадает с минимальным множеством исходной групшы $G$, т.е. supp $\mu=E(G)$; выполняется равенство $\mu\left(\left[0, \gamma_{1}(0)\right)\right)=1$.

Определим отображение $\eta(t)=\widetilde{\mu}([0, t))$, где

$$
\widetilde{\mu}([0, t))= \begin{cases}\mu([0, t)), & t \geqslant 0, \\ -\mu((t, 0)), & t<0 .\end{cases}
$$

Отображение $\eta$ является непрерьвньм, монотонно возрастаюшим и $\eta(\mathbb{R})=\mathbb{R}$.

Для любых $t \in \mathbb{R}$ и $\gamma \in \mathscr{J}$ сравним значения $\eta(\gamma(t))$ и $\eta\left(\gamma\left(\gamma_{1}(t)\right)\right)$. Очевидно, что

$$
\eta(\gamma(t))=\widetilde{\mu}([0, \gamma(t))), \quad \eta\left(\gamma\left(\gamma_{1}(t)\right)\right)=\widetilde{\mu}\left(\left[0, \gamma\left(\gamma_{1}(t)\right)\right)\right)
$$

Тогда

$$
\begin{aligned}
\eta\left(\gamma\left(\gamma_{1}(t)\right)\right) & =\widetilde{\mu}\left(\left[0, \gamma\left(\gamma_{1}(t)\right)\right)\right)=\widetilde{\mu}([0, \gamma(t)))+\mu\left(\left[\gamma(t), \gamma\left(\gamma_{1}(t)\right)\right)\right) \\
& =\eta(\gamma(t))+\gamma_{*}^{-1} \mu\left(\left[t, \gamma_{1}(t)\right)\right) .
\end{aligned}
$$

По предложению $5.4 \tau_{\gamma_{*}^{-1}} \mu, \widetilde{\Gamma}_{1}=c\left(\gamma^{-1}\right) \tau_{\mu, \widetilde{\Gamma}_{1}}$. В частности, из этого равенства в силу определения характера $\tau_{\mu, \widetilde{\Gamma}_{1}}$ и того же предложения 5.4 следует, что для любых $t \in \mathbb{R}$ и $\gamma \in \mathscr{J}$ справедливо равенство

$$
\gamma_{*}^{-1} \mu\left(\left[t, \gamma_{1}(t)\right)\right)=c\left(\gamma^{-1}\right) \mu\left(\left[t, \gamma_{1}(t)\right)\right)
$$


Из условия $G=C_{G}$ следует, что $\mathscr{J}=C_{\mathscr{J}}$. Из нормальности подгрупшы $\widetilde{\Gamma}_{1}$ в групе $G$ следует, что $\widetilde{\Gamma}_{1}$ будет нормальной и в групше $\mathscr{J}$. Так как $\mu\left(\left[0, \gamma_{1}(0)\right)\right)=1$, то характер $\tau_{\mu, \widetilde{\Gamma}_{1}}$ будет нетривиальньм. Тогда по предложению 5.6 , в силу условия $\mathscr{J}=C_{\mathscr{J}}$, характер $A_{\mathscr{J}, \widetilde{\Gamma}_{1}}$ тривиальньй, т.е. $A_{\mathscr{J}, \widetilde{\Gamma}_{1}}=0$. Из тривиальности характера $A_{\mathscr{J}, \widetilde{\Gamma}_{1}}$ следует, что для любого $\gamma \in \mathscr{J}$ коэффициент $c\left(\gamma^{-1}\right)$ равен единище, т.е. мы получаем равенство

$$
\gamma_{*}^{-1} \mu\left(\left[t, \gamma_{1}(t)\right)\right)=\mu\left(\left[t, \gamma_{1}(t)\right)\right),
$$

справедливое для любых $\gamma \in \mathscr{J}$ и любых $t \in \mathbb{R}$.

$\mathrm{C}$ другой стороны, в силу предложения 5.4 для любых $t \in \mathbb{R}$ имеет место равенство

$$
\mu\left(\left[t, \gamma_{1}(t)\right)\right)=\mu\left(\left[0, \gamma_{1}(0)\right)\right) .
$$

Окончательно получим, что для любых $\gamma \in \mathscr{J}$ и любых $t \in \mathbb{R}$ справедливо равенство

$$
\gamma_{*}^{-1} \mu\left(\left[t, \gamma_{1}(t)\right)\right)=1 .
$$

Таким образом, для любых $\gamma \in \mathscr{J}$ и любых $t \in \mathbb{R}$

$$
\eta\left(\gamma\left(\gamma_{1}(t)\right)\right)=\eta(\gamma(t))+1
$$

и, в частности, $\eta\left(\gamma_{1}(t)\right)=\eta(t)+1$.

Определим группу $* \mathscr{J}$ гомеоморфизмов прямой, сохраняюших ориентацию, следуюшим образом:

$$
\begin{array}{r}
* \mathscr{J}=\left\{* \gamma:{ }_{*} \gamma \in \mathrm{Homeo}_{+}(\mathbb{R}) ; \text { сушествует } \gamma \in \mathscr{J}\right. \text { такой, } \\
\text { что для любого } \left.t \in \mathbb{R},{ }_{*} \gamma(\eta(t))=\eta(\gamma(t))\right\} .
\end{array}
$$

Отображение $\eta^{\#}: \mathscr{J} \rightarrow{ }_{*} \mathscr{J}$, где $\eta^{\#}(\gamma)={ }_{*} \gamma,{ }_{*} \gamma(\eta(t))=\eta(\gamma(t)), t \in \mathbb{R}$, является эпиморфизмом. При этом $\operatorname{ker} \eta^{\#}=H_{G}$ и $\eta^{\#}\left(\gamma_{1}\right)={ }_{*} \gamma_{1},{ }_{*} \gamma_{1}(t) \equiv t+1$. Заметим, что

$$
{ }_{*} \gamma(\eta(t)+1)={ }_{*} \gamma\left(\eta\left(\gamma_{1}(t)\right)\right)=\eta\left(\gamma\left(\gamma_{1}(t)\right)\right)=\eta(\gamma(t))+1={ }_{*} \gamma(\eta(t))+1,
$$

откуда следует, что любой элемент $* \gamma \in{ }_{*} \mathscr{J}$ перестановочен с элементом $* \gamma_{1}$.

В силу изоморфизма $\mathscr{J} / H_{G} \simeq\left\langle\widetilde{\Gamma}_{1} / H_{G}, \widetilde{\Gamma}_{1} / H_{G}\right\rangle$ и определения эпиморфизма $\eta^{\#}$ следует, что

$$
* \mathscr{J}=\left\langle{ }_{*} \gamma_{1}, * \gamma_{2}\right\rangle, \text { где } * \gamma_{1}=\eta^{\#}\left(\gamma_{1}\right), \quad * \gamma_{2}=\eta^{\#}\left(\gamma_{2}\right) .
$$

Мы уже отметили, что $* \gamma_{1}$ и ${ }_{*} \gamma_{2}$ будут перестановочными и при этом $* \gamma_{1}(t) \equiv t+1$. Тогда $* \mathscr{J} \in \widetilde{\mathrm{Homeo}_{+}}\left(S^{1}\right)$. По следствию 5.1 для групы $* \mathscr{J}$ существует инвариантная мера $* \nu$. Так как $\eta(t)$ - монотонно возрастающее отображение; то по мере ${ }_{*} \nu$ естественным образом может быть построена мера $\nu$, инвариантная относительно группы $\mathscr{J}$. Из нормальности подгрупп $\widetilde{\Gamma}_{1}$ и $\widetilde{\Gamma}_{2}$ в исходной группе $G$ следует, что и подгруппа $\mathscr{J}$ нормальна в групе $G$. Таким образом, мы построили нормальную подгруппу $\mathscr{J}$ с инвариантной мерой, которая содержит в себе 0-максимальную собственную подгрупу $\widetilde{\Gamma}_{1}$. Противоречие. Следовательно, 0-максимальная подгруппа единственная.

Наконец, из единственности 0-максимальной подгруппы и утверждения шага 3 следует последний пункт теоремы.

Если $G \subseteq \widetilde{\text { Нотео }_{+}}\left(S^{1}\right)$, то теорема 7.1 может быть уточнена. Напомним, что для груп $G \subseteq \widetilde{\text { Нотео }_{+}}\left(S^{1}\right)$ минимальное множество всегда непусто, а из существования проективно-инвариантной меры следует существование инвариантной меры. 
Tеорема 7.2. Пусть $G \subseteq \widetilde{\text { Homeо }_{+}}\left(S^{1}\right) . \quad$ Тогда для группы $G$ выполняется условие $C_{G}=G$ и существует, причем единственная, 0-максимальная подгруппа $M_{0, G}$, при этом она

1) совпадает с группой $G$, если для группь $G$ существует инвариантная мера;

2) содержит нормальную подгруппу $H_{G}$, если для группь $G$ не существует инвариантной меры; более того, $M_{0, G}^{s}=H_{G}$ и фактор-группа $M_{0, G} / H_{G}$ либо тривиальная, либо бесконечная ииклическая группа.

Более того, любая нормальная подгруппа с инвариантной мерой содержится в 0-максимальной подгруппе.

Из определения подгруппы $H_{G}$ и теоремы 7.1 следует простое замечание.

ЗАмечание 7.1. Пусть $G \subseteq$ Ноmео+ $_{+}(\mathbb{R})$ и сушествует непустое минимальное множество. Тогда для 0-максимальной подгруппы $M_{0, G}$ справедливо условие $M_{0, G}^{s}=H_{G}$.

В силу замечания 7.1 имеет место включение $H_{G} \subseteq M_{0, G}$. Выясним, при каких условиях имеет место строгое включение, т.е. $M_{0, G} \neq H_{G}$.

ПРЕДЛОЖЕНИЕ $7.1[16]$. Пусть $G \subseteq$ Homeо $_{+}(\mathbb{R})$. Для существования непусто$2 о$ минимального множества и 0 -максимальной подгруппь $M_{0, G}$ со свойством $M_{0, G} \neq H_{G}$ необходимо и достаточно, чтобы существовала нормальная подгруппа $\Gamma$, для которой фактор-группа $\Gamma /\left\langle\Gamma^{s}\right\rangle$ нетривиальная.

ДокАЗАТЕльство. Необходимость. Пусть существует непустое минимальное множество, а 0-максимальная подгруппа $M_{0, G}$ удовлетворяет условию $M_{0, G} \neq H_{G}$. Так как по замечанию 7.1 выполняется условие $M_{0, G}^{s}=H_{G}$, то достаточно положить $\Gamma=M_{0, G}$.

Достаточность. Пусть существует нормальная подгруппа $\Gamma$, для которой факторгруппа $\Gamma /\left\langle\Gamma^{s}\right\rangle$ нетривиальная. Тогда для такой подгрупшы существует свободно действующий элемент. По теореме 3.3 (п. в)) для групшы $G$ существует непустое минимальное множество. Более того, по теореме 5.2 для такой группы существует инвариантная мера. Следовательно, по теореме 7.1 такая подгруппа содержится в 0-максимальной подгруппе $M_{0, G}$. Так как $\Gamma /\left\langle\Gamma^{s}\right\rangle$ нетривиальная и по замечанию $7.1 M_{0, G}^{s}=$ $H_{G}$, то будет вьполняться условие $M_{0, G} \neq H_{G}$.

В работе [16] условие “фактор-группа $\Gamma /\left\langle\Gamma^{s}\right\rangle$ нетривиальная”, фигурирующее в предложении 7.1, заменено на эквивалентное условие “для подгрупшы $Г$ сушествует инвариантная мера и свободно действующий элемент” (см. лемму 5.1).

Теперь мы можем сформулировать теорему о 0-максимальной подгрупе для группы $G \subseteq \mathrm{Homeo}_{+}\left(S^{1}\right)$.

Tеорема 7.3. Пусть $G \subseteq$ Homeо $_{+}\left(S^{1}\right)$. Тогда для группь $G$ существует, причем единственная, 0-максимальная подгруппа $M_{0, G}$, при этом она

1) совпадает с группой $G$, если для группь $G$ существует инвариантная вероятностная мера;

2) содержит нормальную подгруппу $H_{G}$, если для группы $G$ не существует инвариантной вероятностной меры; более того, $M_{0, G}^{s}=H_{G}$ и фактор-группа $M_{0, G} / H_{G}$ либо тривиальная, либо чиклическая группа конечного порядка. 
Более того, любая нормальная подгруппа с инвариантной вероятностной мерой содержится в 0-максимальной подгруппе.

ДокАЗАТЕЛЬСТво. Если для группы $G$ сушествует инвариантная вероятностная мера, то доказательство очевидно.

Предположим, что для группы $G$ не существует инвариантной вероятностной меры. Пусть группа $\widehat{G}$ и отображения $\bar{g}, \lambda, \pi_{\widehat{G}}$ те же, что и в $\S 1$ (см. предложение 1.1$)$. Несложно заметить, что множества $\mathrm{Fix} \widehat{G^{s}}$ и $\mathrm{Fix} G^{s}$ непусты одновременно и при отображении $\lambda$ образ множества Fix $\widehat{G^{s}}$ совпадает с множеством Fix $G^{s}$. Тогда по теореме 5.1 для группы $\widehat{G}$ инвариантная борелевская мера, конечная на компактах, существует тогда и только тогда, когда для групшы $G$ существует вероятностная мера. Следовательно, для групшы $\widehat{G}$ не существует инвариантной меры. В силу рассуждений, приведенных вьше, для любой нормальной подгруппы $Q \subseteq G$ с инвариантной вероятностной мерой подгруппа $\widehat{Q} \subseteq \widehat{G}$, где $\widehat{Q}=\pi_{\widehat{G}}^{-1}(Q)$, также является нормальной и для нее сушествует инвариантная борелевская мера, конечная на компактах. Более того, нормальная подгрупа $M_{0, G} \subseteq G$ является 0-максимальной тогда и только тогда, когда подгруппа $M_{0, \widehat{G}} \subseteq \widehat{G}$, где $M_{0, \widehat{G}}=\pi_{\widehat{G}}^{-1}\left(M_{0, G}\right)$, является 0-максимальной.

Так как група $\widehat{G}$ принадлежит $\widehat{\text { Homeo }_{+}}\left(S^{1}\right)$, то для нее справедлива теорема 7.2. Поэтому для групш $\widehat{G}$ сушествует 0-максимальная подгруппа, а любая нормальная подгруппа с инвариантной борелевской мерой, конечной на компактах, содержится в 0 -максимальной подгрупше. По той же теореме 7.2 , в силу условий $H_{\widehat{G}}=\pi_{\widehat{G}}^{-1}\left(H_{G}\right)$, $M_{0, \widehat{G}}=\pi_{\widehat{G}}^{-1}\left(M_{0, G}\right), M_{0, \widehat{G}}^{s}=\pi_{\widehat{G}}^{-1}\left(M_{0, G}^{s}\right)$, будет вьполняться условие $M_{0, G}^{s}=H_{G}$.

Остается показать, что фактор-группа $M_{0, G} / H_{G}$ либо тривиальная, либо шиклическая группа конечного порядка. Но по теореме 7.2 фактор-группа $M_{0, \widehat{G}} / H_{\widehat{G}}-$ либо тривиальная, либо бесконечная циклическая группа. Так как минимальное множество группы $\widehat{G}$ является прообразом минимального множества групшы $G$ при отображении $\lambda$, то оно будет инвариантно относительно сдвига $\bar{g}(t)=t+1$. Поэтому для групшы $\Gamma=\left\langle\bar{g}, H_{\widehat{G}}\right\rangle$ будет вьполняться условие Fix $\Gamma^{s} \neq \varnothing$. Тогда по теореме 5.1 для такой групшы существует инвариантная мера и в силу этого она содержится в 0-максимальной подгруппе $M_{0, \widehat{G}}$. Следовательно, элемент $\bar{g}(t)=t+1$ принадлежит группе $M_{0, \widehat{G}}$. Поэтому фактор-группа $M_{0, \widehat{G}} / H_{\widehat{G}}$ всегда бесконечная циклическая группа. Пусть левый смежный класс $\widehat{g} H_{\widehat{G}}$ определяет образуюшую фактор-групшы $M_{0, \widehat{G}} / H_{\widehat{G}}$. Тогда найдется натуральное $n$ такое, что $\widehat{g}^{n} H_{\widehat{G}}=\bar{g} H_{\widehat{G}}$. Гомоморфизм $\pi_{\widehat{G}}: \widehat{G} \rightarrow G$ индуцирует естественньй гомоморфизм фактор-групп $M_{0, \widehat{G}} / H_{\widehat{G}} \rightarrow M_{0, G} / H_{G}$, при котором левьй смежньй класс $\bar{g} H_{\widehat{G}}$ переходит в $H_{G}$. С ледовательно, фактор-группа $M_{0, G} / H_{G}$ является циклической группй конечного порядка.

Из определения подгруппы $H_{G}$ и теоремы 7.3 следует аналог замечания 7.1.

ЗАмечание 7.2. Пусть $G \subseteq$ Homeo+ $_{+}\left(S^{1}\right)$. Тогда для 0-максимальной подгрупшы $M_{0, G}$ справедливо условие $M_{0, G}^{s}=H_{G}$.

В силу замечания 7.2 имеет место включение $H_{G} \subseteq M_{0, G}$. Выясним, при каких условиях имеет место строгое включение, т.е. $M_{0, G} \neq H_{G}$.

ПРЕДЛОЖЕНИЕ 7.2 [16]. Пусть $G \subseteq$ Homeо+ $_{+}\left(S^{1}\right)$. Для существования 0-максимальной подгруппь $M_{0, G}$ со свойством $M_{0, G} \neq H_{G}$ необходимо и достаточ- 
но, чтобы существовала нормальная подгруппа $Г$, для которой фактор-группа $\Gamma /\left\langle\Gamma^{s}\right\rangle$ нетривиальная.

ДокАЗАТЕльство. Необходимость. Пусть 0-максимальная подгруппа $M_{0, G}$ удовлетворяет условию $M_{0, G} \neq H_{G}$. Так как по замечанию 7.2 выполняется условие $M_{0, G}^{s}=H_{G}$, то достаточно положить $\Gamma=M_{0, G}$.

Достаточность. Пусть существует нормальная подгруппа $\Gamma$, для которой факторгруппа $\Gamma /\left\langle\Gamma^{s}\right\rangle$ нетривиальная. Тогда для такой подгруппы существует свободно действующий элемент. Более того, по теореме 5.4 для такой групшы сушествует инвариантная мера. Следовательно, по теореме 7.3 такая подгруппа содержится в 0-максимальной подгруппе $M_{0, G}$, для которой будет выполняться условие $M_{0, G} \neq H_{G}$.

Перейдем к описанию 1-максимальных подгрупп для груп $G \subseteq \mathrm{Homeo}_{+}(\mathbb{R})$.

ТеОрема 7.4. Пусть $G \subseteq$ Homeo+ $_{+}(\mathbb{R})$ и для группь $G$ существует непустое минимальное множество. Тогда для группь $G$ существует единственная 1-максимальная подгруппа $M_{1, G}$, любая нормальная подгруппа с проективно-инвариантной мерой содерәится в подгруппе $M_{1, G}$, а подгруппа $M_{1, G}$

1) совпадает с әруппой $G$, если для группьи $G$ существует проективно-инвариантная мера;

2) совпадает с 0-максимальной подгруппой $M_{0, G}$, если для группь $G$ не существует проективно-инвариантной мерь.

ДокАЗАТЕльство. Если для группы $G$ существует проективно-инвариантная мера, то доказательство очевидно.

Пусть для группы $G$ не сушествует проективно-инвариантной меры. Покажем, что для любой нормальной подгруппы $Q \subseteq G$ с проективно-инвариантной мерой $\mu$ существует инвариантная мера. Действительно, предположим, что это не так. Тогда по теореме 6.5 групша $Q$ будет проективно-строго эргодичной. Для любого элемента $g \in G$ в силу нормальности подгрупш $Q$ мера $g_{*} \mu$ также будет проективно-инвариантной относительно групшы $Q$ (определение меры $g_{*} \mu$ см. в $\S 7$ ). Так как группа $Q$ проективно-строго эргодична, то найдется число $c(g)>0$ такое, что $g_{*} \mu=c(g) \mu$. Поэтому мера $\mu$ оказывается проективно-инвариантной относительно исходной группы $G$. Противоречие. С ледовательно, для любой нормальной подгруппы $Q \subseteq G$ с проективно-инвариантной мерой существует инвариантная мера. Но для таких групп 1-максимальная подгруппа совпадает с 0-максимальной подгруппой, и теорема доказана.

Мы уже отмечали, что для подгруп группы $\widetilde{\text { оотео }_{+}}\left(S^{1}\right)$ из сушествования проективно-инвариантной меры следует существование инвариантной меры, а для подгрупп групшы Ноmео+ $\left(S^{1}\right)$ всякая проективно-инвариантная мера является инвариантной. Поэтому для таких подгруп уточнение теоремы 7.4 либо формулировка ее аналогов не требуется.

Итак, для групा $G \subseteq$ Homeо+ $(\mathbb{R})$ с непустым минимальньм множеством существует, причем единственная, 0-максимальная подгруппа $M_{0, G}$, для которой справедливы условия:

$$
H_{G} \subseteq M_{0, G} \subseteq G, \quad M_{0, G}^{s}=H_{G}
$$


По теореме о фактор-групе (теорема 2.1) $M_{0, G} / H_{G}$ является коммутативной группой. Поэтому сложность структуры таких групп $G$ зависит от сложности факторгрупшы $G / M_{0, G}$.

Tеорема 7.5. Пусть $G \subseteq$ Homeo+ $_{+}(\mathbb{R})$ и для нее существует непустое минимальное множество. Тогда справедливо одно из следующих взаимоисключающих условий:

1) для группь $G$ существует инвариантная мера и при этом фактор-группа $G / M_{0, G}$ тривиальная, а фактор-группа $M_{0, G} / H_{G}$ коммутативная;

2) для группь $G$ существует проективно-инвариантная мера, но не существует инвариантной мерь и при этом фактор-группь $G / M_{0, G}$, $M_{0, G} / H_{G}$ нетривиальнье и коммутативнье, а фактор-группа $M_{0, G} / H_{G}$ нециклическая;

3) для группь $G$ не существует проективно-инвариантной меры, $G=C_{G}$ и при этом фактор-группа $G / M_{0, G}$ некоммутативная, а фактор-группа $M_{0, G} / H_{G}$ либо тривиальная, либо бесконечная циклическая группа;

4) для группь $G$ не существует проективно-инвариантной мерьи, $G \neq C_{G}$ и при этом фактор-группа $G / M_{0, G}$ некоммутативная, а фактор-группа $M_{0, G} / H_{G}$ тривиальная.

ДокАЗАТЕльство. Пункт 1). Пусть для группы $G$ сушествует инвариантная меpa. Тогда из определения 0-максимальной подгрупшы следует, что $G=M_{0, G}$ и фактор-группа $G / M_{0, G}$ тривиальная. В таком случае из определения подгрупшы $H_{G}$ следует, что $H_{G}=G^{s}$ и по теореме о фактор-групше (теорема 2.1 ) $M_{0, G} / H_{G}$ коммутативная.

Пункт 2). Пусть для группы $G$ существует проективно-инвариантная мера $\mu$, но не сушествует инвариантной меры. По теореме $7.1 M_{0, G}=C_{G}$, а по лемме $6.3 \mathscr{H}_{\mu, G}=$ $H_{G}$ и $\mathscr{C}_{\mu, G}=C_{G}$. Тогда коммутативность фактор-групп $G / M_{0, G}, M_{0, G} / H_{G}$ будет следовать из теоремы 6.1 , а их нетривиальность и нецикличность $M_{0, G} / H_{G}$ - из леммы 6.1.

Пункты 3$)$ и 4). Пусть для групшы $G$ не существует проективно-инвариантной меры. Для таких групп цикличность фактор-групшы $M_{0, G} / H_{G}$ в случае $G=C_{G}$ и ее тривиальность в случае $G \neq C_{G}$ следует из теоремы 7.1 . Нам остается доказать некоммутативность фактор-групшы $G / M_{0, G}$. Заметим, что для групшы $G$ в силу теоремы 3.1 минимальное множество $E(G)$ недискретное и единственное. Доказательство проведем от противного.

Предположим, что фактор-группа $G / M_{0, G}$ коммутативная. Пусть $\mu$ является инвариантной мерой для 0-максимальной подгруппы $M_{0, G}$. Так как $M_{0, G} / H_{G}$ шиклическая, то мы можем так переопределить меру $\mu$, чтобы она удовлетворяла условиям: $\mu$ является инвариантной относительно $M_{0, G}$; мера $\mu$ непрерывная, и ее носитель совпадает с минимальным множеством исходной группы $G$, т.e. $\operatorname{supp} \mu=E(G)$. При этом фактор-группа $M_{0, G} / H_{G}$ нетривиальная, а левьй смежный класс $m H_{G}$ определяет ее образуюшую. Не нарушая обшности, можем считать, что для любого $t \in \mathbb{R}$ вьполняется условие $m(t)>t$. Более того, меру $\mu$ можно так подобрать, чтобы в дополнение к перечисленным условиям она удовлетворяла и условию $\mu([0, m(0)))=1$. 
Определим отображение $\eta(t)=\widetilde{\mu}([0, t))$, где

$$
\widetilde{\mu}([0, t))= \begin{cases}\mu([0, t)), & t \geqslant 0 \\ -\mu((t, 0]), & t<0 .\end{cases}
$$

Отображение $\eta$ является непрерьвньм, монотонно возрастаюшим и $\eta(\mathbb{R})=\mathbb{R}$.

Определим группу $* G$ гомеоморфизмов прямой, сохраняюших ориентацию, следуюшим образом: ${ }_{*} G=\left\{{ }_{*} g:{ }_{*} g \in \mathrm{Homeo}_{+}(\mathbb{R})\right.$; сушествует $g \in G$ такой, что ${ }_{*} g(\eta(t))=\eta(g(t))$ для любого $\left.t \in \mathbb{R}\right\}$. Отображение $\eta^{\#}: G \rightarrow{ }_{*} G$, где $\eta^{\#}(g)={ }_{*} g$, ${ }_{*} g(\eta(t))=\eta(g(t)), t \in \mathbb{R}$, является эпиморфизмом и $\operatorname{ker} \eta^{\#}=H_{G}$. При этом если фактор-группа $M_{0, G} / H_{G}$ не тривиальная, то $\eta^{\#}(m)={ }_{*} m,{ }_{*} m(t) \equiv t+1$. Из определения групшы $* G$ следует, что минимальное множество $E\left({ }_{*} G\right)$ групшы $* G$ совпадает с $\mathbb{R}$, т.е. $E\left({ }_{*} G\right)=\mathbb{R}$. Рассмотрим два случая.

Случай первый - фактор-группа $M_{0, G} / H_{G}$ тривиальная. Тогда фактор-группа $G / H_{G}$ также будет коммутативной. Так как $\operatorname{ker} \eta^{\#}=H_{G}$, то группа ${ }_{*} G$ изоморфна фактор-групше $G / H_{G}$ и, соответственно, коммутативна. Из коммутативности групшы гомеоморфизмов ${ }_{*} G$ следует, что для любого элемента ${ }_{*} g \in{ }_{*} G^{s}$ множество $\mathrm{Fix}\left\langle{ }_{*} g\right\rangle$ инвариантно относительно группы ${ }_{*} G$. В таком случае по теореме 3.1 справедливо включение $\mathbb{R}=E\left({ }_{*} G\right) \subseteq \operatorname{Fix}\left\langle{ }_{*} g\right\rangle$. Из справедливости такого включения для любого элемента ${ }_{*} g \in{ }_{*} G^{s}$ следует условие ${ }_{*} G^{s}=\langle e\rangle$, что эквивалентно условию $\mathrm{Fix}{ }_{*} G^{s}=$ $\mathbb{R}$. Тогда по теореме 5.1 для группы $* G$ сушествует инвариантная мера $* \nu$. Определим на $\mathbb{R}$ меру $\nu$ по следующему правилу: для любых $t_{1}, t_{2} \in \mathbb{R}, t_{2} \geqslant t_{1}$,

$$
\nu\left(\left[t_{1}, t_{2}\right)\right)={ }_{*} \nu\left(\left[\eta\left(t_{1}\right), \eta\left(t_{2}\right)\right)\right) .
$$

Несложно видеть, что такое определение меры $\nu$ является корректным и носитель меры $\nu$ принадлежит минимальному множеству $E(G)$. Более того, в силу справедливости условия ${ }_{*} g(\eta(t))=\eta(g(t))$ для любых $g \in G,{ }_{*} g=\eta^{\#}(g), t \in \mathbb{R}$, мера $\nu$ будет инвариантной относительно исходной групшы $G$. Противоречие. Следовательно, фактор-группа $G / M_{0, G}$ некоммутативная.

Случай второй $-M_{0, G} / H_{G}$ нетривиальная. По теореме 7.1 для такой группы $G$ имеет место условие $G=C_{G}$. Для любых $t \in \mathbb{R}, g \in G$ сравним значения $\eta(g(t))$ и $\eta(g(m(t)))$. Очевидно, что

$$
\eta(g(t))=\widetilde{\mu}([0, g(t))), \quad \eta(g(m(t)))=\widetilde{\mu}([0, g(m(t)))) .
$$

Тогда

$$
\begin{aligned}
\eta(g(m(t))) & =\widetilde{\mu}([0, g(m(t))))=\widetilde{\mu}([0, g(t)))+\mu([g(t), g(m(t)))) \\
& =\eta(g(t))+g_{*}^{-1} \mu([t, m(t)))
\end{aligned}
$$

По предложению $5.4 \tau_{g_{*}^{-1} \mu, M_{0, G}}=c\left(g^{-1}\right) \tau_{\mu, M_{0, G}}$. В частности, из этого равенства в силу определения характера $\tau_{\mu, M_{0, G}}$ и того же предложения 5.4 следует, что для любых $t \in \mathbb{R}$ и $g \in G$ справедливо равенство

$$
g_{*}^{-1} \mu([t, m(t)))=c\left(g^{-1}\right) \mu([t, m(t))) .
$$


Так как $\mu([0, m(0)))=1$, то характер $\tau_{\mu, M_{0, G}}$ будет нетривиальным. Тогда по предложению 5.6, в силу условия $G=C_{G}$, характер $A_{G, M_{0, G}}$ будет тривиальным, т.е. $A_{G, M_{0, G}}=0$. Из тривиальности характера $A_{G, M_{0, G}}$ следует, что для любого $g \in G$ коэфрициент $c\left(g^{-1}\right)$ равен единище, т.е. мы получаем равенство

$$
g_{*}^{-1} \mu([t, m(t)))=\mu([t, m(t))),
$$

справедливое для любых $g \in G$ и $t \in \mathbb{R}$. C другой стороны, в силу предложения 5.4 для любых $t \in \mathbb{R}$ имеет место равенство

$$
\mu([t, m(t)))=\mu([0, m(0))) .
$$

Окончательно получим, что любых $g \in G$ и $t \in \mathbb{R}$ справедливо равенство

$$
g_{*}^{-1} \mu([t, m(t)))=1
$$

Таким образом, для любых $g \in G$ и $t \in \mathbb{R}$

$$
\eta(g(m(t)))=\eta(g(t))+1
$$

и, в частности, $\eta(m(t))=\eta(t)+1$. Заметим, что

$$
{ }_{*} g(\eta(t)+1)={ }_{*} g(\eta(m(t)))=\eta(g(m(t)))=\eta(g(t))+1={ }_{*} g(\eta(t))+1,
$$

откуда следует, что любой элемент ${ }_{*} g \in{ }_{*} G$ перестановочен с элементом ${ }_{*} m(t) \equiv t+1$. Следовательно, группа ${ }_{*} G$ содержится в $\widehat{\text { Нотео }}_{+}\left(S^{1}\right)$. Несложно заметить, что подгруппа $M_{0,{ }_{*} G}=\eta^{\#}\left(M_{0, G}\right)$ совпадает с циклической подгруппой $\left.{ }_{*} m\right\rangle$ и, как было доказано вьше, принадлежит центру группы ${ }_{*} G$. Так как $\operatorname{ker} \eta^{\#}=H_{G}$ и $M_{0,{ }_{*} G}=$ $\eta^{\#}\left(M_{0, G}\right)$, то группа $* G$ изоморфна фактор-групе $G / H_{G}$, а фактор-группа ${ }_{*} G / M_{0,{ }_{*} G}$ изоморфна фактор-группе $G / M_{0, G}$. В таком случае фактор-группа ${ }_{*} G / M_{0,{ }_{*} G}$ также будет коммутативной. Из коммутативности фактор-групш ${ }_{*} G / M_{0, *} G$ и принадлежности подгрупшы $M_{0,} G$ к центру группы ${ }_{*} G$ следует, что любой элемент ${ }_{*} g \in{ }_{*} G^{s}$ также будет принадлежать центру группы ${ }_{*} G$. В силу этого для любого элемента ${ }_{*} g \in$ ${ }_{*} G^{s}$ множество $\operatorname{Fix}\left\langle_{*} g\right\rangle$ будет инвариантным относительно групшы $* G$. В таком случае по теореме 3.1 справедливо включение $\mathbb{R}=E\left({ }_{*} G\right) \subseteq \operatorname{Fix}\left\langle_{*} g\right\rangle$. Из справедливости такого включения для любого элемента ${ }_{*} g \in{ }_{*} G^{s}$ следует условие ${ }_{*} G^{s}=\langle e\rangle$, что эквивалентно условию $\operatorname{Fix}_{*} G^{s}=\mathbb{R}$. Тогда по теореме 4.1 такая группа ${ }_{*} G$ является коммутативной, а по теореме 5.1 для групшы ${ }_{*} G$ существует инвариантная мера $* \nu$. Как и в первом случае, по мере $* \nu$ мы можем построить меру $\nu$, инвариантную относительно исходной групшы $G$. Противоречие. Следовательно, фактор-группа $G / M_{0, G}$ некоммутативная.

Для групп $G \subseteq \widetilde{\text { Ноmео }_{+}}\left(S^{1}\right)$ теорема 7.5 допускает уточнения, так как для таких груп минимальное множество непустое, $G=C_{G}$, а из сушествования проективно-инвариантной меры следует сушествование инвариантной меры. 


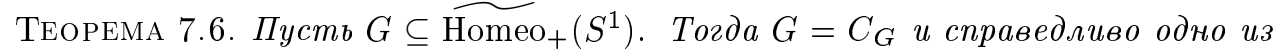
следующ, взаимоисключающ,х условий:

1) для группь $G$ существует инвариантная мера и при этом фактор-группа $G / M_{0, G}$ тривиальная, а фактор-группа $M_{0, G} / H_{G}$ коммутативная;

2) для группь $G$ не существует инвариантной меры и при этом факторгруппа $G / M_{0, G}$ некоммутативная, а фактор-группа $M_{0, G} / H_{G}$ либо тривиальная, либо бесконечная циклическая группа.

Теперь мы в состоянии сфформулировать аналог теоремы 7.5 для груп $G \subseteq$ $\mathrm{HomeO}_{+}\left(S^{1}\right)$.

Теорема 7.7. Пусть $G \subseteq$ Ноmео+ $_{+}\left(S^{1}\right)$. Тогда справедливо одно из следующих взаимоисключающих условий:

1) для группь $G$ существует вероятностная мера и при этом факторгруппа $G / M_{0, G}$ тривиальная, а фактор-группа $M_{0, G} / H_{G}$ коммутативная;

2) для группь $G$ не существует вероятностной меры и при этом факторгруппа $G / M_{0, G}$ некоммутативная, а фактор-группа $M_{0, G} / H_{G}$ либо тривиальная, либо чиклическая группа конечного порядка.

ДокАЗАТЕльство. Пусть группа $\widehat{G}$ и отображения $\bar{g}, \lambda, \pi_{\widehat{G}}$ те же, что и в $\S 1$ (см. предложение 1.1). Несложно заметить, что множества Fix $\widehat{G^{s}}$ и Fix $G^{s}$ непусты одновременно и при отображении $\lambda$ образ множества Fix $\widehat{G^{s}}$ совпадает с множеством Fix $G^{s}$. Тогда по теореме 5.1 для групшы $\widehat{G}$ инвариантная борелевская мера, конечная на компактах, существует тогда и только тогда, когда для групы $G$ сушествует вероятностная мера. Заметим, что $H_{\widehat{G}}=\pi_{\widehat{G}}^{-1}\left(H_{G}\right), M_{0, \widehat{G}}=\pi_{\widehat{G}}^{-1}\left(M_{0, G}\right), M_{0, \widehat{G}}^{s}=$ $\pi_{\widehat{G}}^{-1}\left(M_{0, G}^{s}\right)$. Так как группа $\widehat{G}$ принадлежит $\widehat{\mathrm{Homeo}_{+}}\left(S^{1}\right)$, то для нее справедлива теорема 7.6 .

Если для групш $G$ сушествует инвариантная вероятностная мера, то доказательство очевидньм образом следует из п. 1) теоремы 7.6.

Предположим, что для группы $G$ не сушествует инвариантной вероятностной меры. Тогда для группы $\widehat{G}$ также не существует инвариантной меры. В таком случае некоммутативность фактор-группы $G / M_{0, G}$ следует из некоммутативности фактор-групшы $\widehat{G} / M_{0, \widehat{G}}$. Остается показать, что фактор-группа $M_{0, G} / H_{G}$ либо тривиальная, либо циклическая группа конечного порядка. Но по теореме 7.6 фактор-группа $M_{0, \widehat{G}} / H_{\widehat{G}}$ либо тривиальная, либо бесконечная циклическая групп. Заметим, что элемент $\bar{g}(t)=t+1$ принадлежит группе $M_{0, \widehat{G}}$. Поэтому фактор-група $M_{0, \widehat{G}} / H_{\widehat{G}}$ всегда бесконечная циклическая група. Пусть левый смежный класс $\widehat{g} H_{\widehat{G}}$ определяет образуюшую фактор-группы $M_{0, \widehat{G}} / H_{\widehat{G}}$. Тогда найдется натуральное $n$ такое, что $\widehat{g}^{n} H_{\widehat{G}}=\bar{g} H_{\widehat{G}}$. Гомоморфизм $\pi_{\widehat{G}}: \widehat{G} \rightarrow G$ индуцирует естественньй гомоморфизм фактор-групा $M_{0, \widehat{G}} / H_{\widehat{G}} \rightarrow M_{0, G} / H_{G}$, при котором левый смежньй класс $\bar{g} H_{\widehat{G}}$ переходит в $H_{G}$. Следовательно, фактор-группа $M_{0, G} / H_{G}$ является циклической группй конечного порядка.

Для групп $G$ с непустым минимальным множеством теорема 7.5 в терминах фактор-групп $G / M_{0, G}, M_{0, G} / H_{G}$ дает критерии существования либо отсутствия инва- 
риантных и проективно-инвариантных мер. Как следует из теоремы 7.5, структура выделенных фактор-групп строго определенная и произвольной быть не может.

С другой стороны, для групп с проективно-инвариантной мерой, в силу той же теоремы 7.5 фактор-група $G / H_{G}$ является разрешимой ступени не больше двух. Интересен вопрос о наличии критерия существования проективно-инвариантной меры, сформулированного в терминах фактор-группы $G / H_{G}$. Теорема 7.5 на этот вопрос не отвечает, ибо в случае отсутствия проективно-инвариантной меры мы можем утверждать только лишш, что фактор-групша $G / H_{G}$ некоммутативна. Все эти вопросы будут обсуждены в $\S 9$.

7.2. Топологические признаки существования $\omega$-проективно-инвариантной меры. Одним из первых результатов по метрическим инвариантам является теорема Боголюбова-Крылова о существовании инвариантной меры для гомеоморфизма окружности. Во введении мы отмечали, что по истории вопроса о сушествовании инвариантной меры для групп гомеоморфизмов локально компактного пространства существует исчерпьвающий обзор [2]. Как нами было отмечено, $\omega$-проективно-инвариантные меры являются обобщением проективно-инвариантных мер и, в частности, инвариантных мер. В предыдущих параграфах были подробно исследованы инвариантные и проективно-инвариантные меры: получен ряд критериев сушествования таких мер; описаны носители этих мер; сформулированы теоремы об эргодичности. Здесь будут приведены признаки сушествования этих более общих метрических инвариантов.

ТЕорема 7.8 (обобщенная теорема Боголюбова-Крылова) [11]. Пусть $G \subseteq$ Homeо $_{+}(\mathbb{R})$. Если минимальное множсество группь $G$ непусто, то найдется такое кардинальное число $\omega$, что для группь $G$ существует $\omega$-проективно-инвариантная мера.

Так как для групшы $G \subseteq \widetilde{\text { Нотео }_{+}}\left(S^{1}\right)$ всегда существует минимальное множество, то теорема 7.8 может быть уточнена.

Tеорема 7.9. Пусть $G \subseteq \widetilde{\text { Homeо }_{+}}\left(S^{1}\right)$. Тогда найдется такое кардинальное число $\omega$, что для группь $G$ существует $\omega$-проективно-инвариантная мера.

Теперь мы можем сформулировать теорему о существовании $\omega$-проективно-инвариантной меры для групшы $G \subseteq \mathrm{Homeo}_{+}\left(S^{1}\right)$.

Tеорема 7.10. Пусть $G \subseteq$ Homeо+ $_{+}\left(S^{1}\right)$. Тогда найдется такое кардинальное число $\omega$, что для группь $G$ существует $\omega$-проективно-инвариантная мера.

ДокАзАТЕльство. Пусть группа $\widehat{G}$ и отображения $\bar{g}, \lambda, \pi_{\widehat{G}}$ те же, что и в $\S 1$ (см. предложение 1.1). Так как $\widehat{G} \subset \widehat{\text { Homeо }_{+}}\left(S^{1}\right)$, то найдется такое кардинальное число $\omega$, что для группы $\widehat{G}$ сушествует $\omega$-проективно-инвариантная мера. В работе [11] доказательство существования $\omega$-проективно-инвариантной меры построено на изучении свойств произвольной максимальной цепи вложенных $G$-инвариантных замкнутых выпуклых конусов в пространстве мер $\mathscr{M}^{+}$. Показано, что для любой такой максимальной цепи сушествует $\omega$-проективно-инвариантная мера, принадлежащая всем элементам этой цепи. Очевидно, мы можем рассмотреть такую максимальную цепь, 
стартуя с конуса $\mathscr{K}_{\mu}(\widehat{G})$ (см. определение 7.1$)$, где $\mu$ - некоторая мера, инвариантная относительно сдвига $\bar{g}(t)=t+1$. Так как $\widehat{G} \in \widetilde{\text { Нотео }_{+}}\left(S^{1}\right)$, то все меры из конуса $\mathscr{K}_{\mu}(\widehat{G})$ также будут инвариантны относительно сдвига $\bar{g}$. Тогда $\omega$-проективно-инвариантная мера $\widehat{\mu}$ также будет инвариантна относительно сдвига $\bar{g}$. Несложно заметить, что ограничение меры $\widehat{\mu}$ на полуинтервал $[0,1)$ будет задавать $\omega$-проективно-инвариантную меру для исходной группы $G$.

Здесь интересен следующий вопрос. В каких случаях существует $\omega$-проективно-инвариантная мера с конечным $\omega$ ? Ответ на этот вопрос дает следующая теорема.

TеOPема 7.11. Пусть $G \subseteq$ Homeо+ $_{+}(\mathbb{R})$ и для нее существует непустое минимальное множсество. Если для группь $G$ не существует проективно-инвариантной мерьи, то для любой $\omega$-проективно-инвариантной мерь $\mu$ кардинальное число $\omega$ не мохет быть конечным.

ДокАЗАТЕльство. Так как для группы $G$ не существует проективно-инвариантной меры, то минимальное множество $E(G)$ недискретное.

Доказательство проведем от противного. Пусть существует $\omega$-проективно-инвариантная мера $\mu$, для которой кардинальное число $\omega$ конечное. Конечность $\omega$ означает, что орбита $G_{*}(\mu)=\left\{g_{*} \mu: g \in G\right\}$ расположена на конечном числе лучей пространства мер $\mathscr{M}$, т.е. количество лучей $\left\{\lambda g_{*} \mu: \lambda \in \mathbb{R}_{+}\right\}_{g \in G}$ в пространстве $\mathscr{M}$ конечно. Определим нормальную подгруппу $Q$ групш $G$ по следуюшему правилу:

$$
Q=\left\{q: \forall g \in G, q_{*}\left(g_{*} \mu\right)=\gamma_{q g} g_{*} \mu, \gamma_{q g}>0\right\} .
$$

Для подгрупшы $Q_{*}$ крайние лучи $\left\{\lambda g_{*} \mu: \lambda \in \mathbb{R}_{+}\right\}_{g \in G}$ являются инвариантными.

В частности, луч $\left\{\lambda \mu: \lambda \in \mathbb{R}_{+}\right\}$будет инвариантен относительно подгрупшы $Q_{*}$, т.е. мера $\mu$ является проективно-инвариантной относительно подгрупшы $Q$. Тогда по теореме 7.4 подгруппа $Q$ содержится в 0 -максимальной подгруппе $M_{0, G}$. Так как крайних лучей конечное число, то фактор-группа $G / Q$ будет конечной. В таком случае фактор-группа $G / M_{0, G}$ также конечная. По теореме 7.1 (пш. 3) и 4)) фактор-групп $M_{0, G} / H_{G}$ либо тривиальная, либо циклическая. Образующей такой циклической групшы является свободно действуюший гомеоморфизм. Тогда из определения подгрупшы $H_{G}$ следует, что в обоих случаях для любой точки $t \in E(G)$ орбита $G(t)$ будет дискретной, а значит, дискретно и само минимальное множество. Противоречие. Следовательно, кардинальное число $\omega$ не может быть конечным.

Так как для группы $G \subseteq$ Homeo+ $_{+}\left(S^{1}\right)$ всегда сушествует непустое минимальное множество, то теорема 7.11 допускает естественное уточнение.

Теорема 7.12. Пусть $G \subseteq$ Homeо $_{+}\left(S^{1}\right)$. Если для группы $G$ не существует инвариантной мерь, то для любой $\omega$-проективно-инвариантной меры $\mu$ кардинальное число $\omega$ не можсет бить конечным.

ДокАЗАТЕльство. Пусть группа $\widehat{G}$ и отображения $\bar{g}, \lambda, \pi_{\widehat{G}}$ те же, что и в $\S 1$ (см. предложение 1.1). Покажем, что для групшы $\widehat{G}$ не сушествует проективно-инвариантной меры. Действительно, в противном случае в силу предложения 6.5 для такой групш сушествует инвариантная мера $\widetilde{\mu}$. Тогда ограничение меры $\widetilde{\mu}$ на полуинтервал $[0,1)$ будет задавать меру, инвариантную относительно исходной групы $G$. Противоречие. Следовательно, для групшы $\widehat{G}$ не сушествует проективно-инвариантной меры. По мере $\mu$ можно однозначно определить меру $\widehat{\mu}$ на всем $\mathbb{R}$ по следуюшему правилу: 
$\left.\widehat{\mu}\right|_{[0,1]}=\mu ; \widehat{\mu}$ инвариантна относительно сдвига $\bar{g}(t) \equiv t+1$. Несложно видеть, что мера $\widehat{\mu}$ является $\omega$-проективно-инвариантной относительно групш $\widehat{G}$. Поэтому по теореме 7.11 оно не может быть конечным.

У тверждение обобщенной теоремы Боголюбова-Крылова (теорема 7.8) о существовании $\omega$-проективно-инвариантной меры, вероятнее всего, имеет обращение.

ГиПОте3А 7.1. Пусть $G \subseteq$ Homeо $_{+}(\mathbb{R})$. Если для группь $G$ существует $\omega$-проективно-инвариантная мера, то минимальное множество непустое.

Для $\omega$-проективно-инвариантной меры также важно описание как ее носителей, так и ее эргодических свойств. Носители инвариантных и проективно-инвариантных мер нами уже описаны.

ГИпотезА 7.2. Пусть $G \subseteq$ Homeo+ $(\mathbb{R})$, для группь $G$ существует $\omega$-проективно-инвариантная мера $\mu(\omega \neq 1)$, но не существует проективно-инвариантной мерьы. Тогда носитель $\operatorname{supp} \mu$ меры $\mu$ совпадает с минимальным множеством.

ГиПотезА 7.3. Пусть $G \subseteq$ Homeо+ $(\mathbb{R})$, для группь $G$ существует $\omega$-проективно-инвариантная мера $\mu(\omega \neq 1)$, но не существует проективно-инвари-

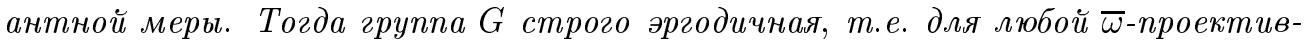
но-инвариантной меры $\bar{\mu}$ справедливо условие $\bar{\mu} \in \mathscr{K}_{\mu}(G)$.

\section{§ 8. Метрические инварианты и полусопряженность}

Важность метрических инвариантов в задаче классификации груп гомеоморфизмов прямой, сохраняюших ориентацию, обуславливает систематическое исследование как вопроса существования таких инвариантов, так и их характеристик. В частности, к таким характеристикам относится определенное в работе [9] понятие полусопряженности, которое оказьвается каноническим объектом теории метрических инвариантов для групп $G \subseteq \operatorname{Homeo}(X)$. Связь такого определения полусопряженности с метрическими инвариантами позволяет поставить вопрос о выделении классов групा гомеоморфизмов прямой, элементам из которых будет полусопряжена заданная группа гомеоморфизмов с непустьм минимальным множеством. В работах [29], [19] приводится другое определение полусопряженности. Взаимосвязи между такими определениями полусопряженности будут обсуждены ниже.

ОПРедЕЛЕниЕ 8.1 [9]. Пусть заданы группы $G,{ }_{*} G \subseteq \mathrm{Homeo}_{+}(\mathbb{R})$. Группа $G$ называется полусопряжсенной группе $* G$, если сушествуют сохраняюшее ориентацию (монотонно возрастающее) отображение $\eta: \mathbb{R} \rightarrow \mathbb{R}$ с образом, состояшим из более чем одной точки, и эпиморфизм $\eta^{\#}: G \rightarrow{ }_{*} G$ такие, что для любого $q \in G$ диаграмма

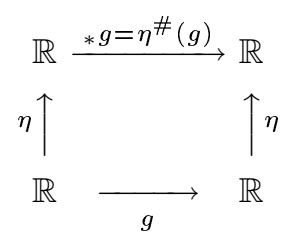

коммутативна, т.е. $\eta^{\#}(g) \eta=\eta g$. 
Если в определении полусопряженности отображение $\eta$ непрерывно, то говорят о топологической полусопряженности. Если отображение $\eta$ является гомеоморфизмом, и как следствие эпиморфизм $\eta^{\#}$ является изоморфизмом, то группа $G$ назьвается топологически сопряженной групе ${ }_{*} G$ или просто сопряженной.

ОПРЕДЕЛЕНИЕ 8.2. Пусть заданы групшы $G,{ }_{*} G \subseteq \operatorname{Homeo}\left(S^{1}\right)$. Группа $G$ назьвается полусопряженной группе ${ }_{*} G$, если:

1) группа $\widehat{G} \subseteq \operatorname{Homeo(}(\mathbb{R})$ полусопряжена группе ${ }_{*} \widehat{G} \subseteq \operatorname{Homeo}(\mathbb{R})$, где $\widehat{G}$ и ${ }_{*} \widehat{G}-$ групшы накрытий для гомеоморфизмов окружности из $G$ и ${ }_{*} G$ соответственно (см. $\S 1)$;

2) отображение $\eta$ из определения 8.1 коммутирует с гомеоморфизмом $\bar{g}(t)=t+1$, т.е. $\eta \bar{g}=\bar{g} \eta$.

Приведем определение полусопряженности из работ [29], [19]. Пусть Г - абстрактная группа. Действием группы $\Gamma$ на $\mathbb{R}$ будем называть гомоморфизм $\Phi: \Gamma \rightarrow \operatorname{Homeo}(\mathbb{R})$. Действие $\Phi$ называется полусопряженным действию * $\Phi$, если существует сохраняющее ориентащию (монотонно возрастающее) отображение $\eta: \mathbb{R} \rightarrow \mathbb{R}$ такое, что образ состоит из более чем одной точки и для любого $\gamma \in \Gamma$ справедливо правило перестановочности $\eta \Phi(\gamma)={ }_{*} \Phi(\gamma) \eta$. Такое определение полусопряженности позволяет сравнивать алгебраические свойства подгрупп группы Ноmeо $(\mathbb{R})$ с соответствуюшими свойствами выделенной абстрактной групшы Г. Более того, Э. Гизом установлено, что такая полусопряженность задает отношение эквивалентности. Если гомоморфизм $\Phi$ является мономорфизмом, то, отождествляя групу $\Gamma$ с ее образом $\operatorname{Im} \Phi$, получим понятие полусопряженности из определения 8.1. Мы уже отмечали, что полусопряженность из определения 8.1 важна тем, что является каноническим объектом теории метрических инвариантов, что и будет продемонстрировано в теоремах 8.1 и 8.3.

Далее в терминах полусопряженности для груп $G \subseteq \mathrm{Homeo}_{+}(X)$ (теоремы 8.1, 8.2) и групा $G \subseteq \operatorname{Homeo}(X)$ (теоремы 8.4, 8.5) будут сфформулированы критерии существования инвариантных мер, а также проективно-инвариантных мер (теоремы 8.3, 8.6). Приводится один признак полусопряженности групшы $G \subseteq \mathrm{Homeo}_{+}(\mathbb{R})$ группе аф̆финных преобразований прямой (теорема 8.7). Для группы $G \subseteq \mathrm{Homeo}_{+}(\mathbb{R})$ сформулирован критерий ее сопряженности некоторой групе ${ }_{*} G \subseteq \widehat{\text { Homeo+ }_{+}}\left(S^{1}\right)$ (предложение 8.1). Даются эквивалентные переформулировки теорем 8.4-8.6. Такие переформулировки (теоремы $8.4^{\prime}-8.6^{\prime}$ ) позволяют сформулировать соответствующую гипотезу для групп с $\omega$-проективно-инвариантной мерой.

Теорема 8.1 [9]. Пусть $G \subseteq$ Hотео+ $_{+}(\mathbb{R})$. Тогда следующие утвержсдения эквивалентни:

(1) существует борелевская мера $\mu$, конечная на компактах и инвариантная относительно группьи $G$;

(2) әруппа $G$ полусопряжснна некоторой группе ${ }_{*} G \subseteq \mathrm{Homeo}_{+}(\mathbb{R})$ сдвигов на прямой.

Сформулируем аналог теоремы 8.1 для груп, действующих на окружности.

ТеОрема 8.2. Пусть $G \subseteq$ Нотео+ $_{+}\left(S^{1}\right)$. Тогда следующие утвержсдения әквивалентнь:

(1) существует вероятностная мера $\mu$, инвариантная относительно группь $G$; 
(2) группа $G$ полусопряжена некоторой группе ${ }_{*} G \subseteq \mathrm{Homeo}_{+}\left(S^{1}\right)$ вращений окружности.

ДокАЗАТЕЛЬСтво. Пусть група $\widehat{G} \subseteq$ Hотео+ $_{+}\left(S^{1}\right)$ и отображения $\bar{g}, \lambda, \pi_{\widehat{G}}$ те же, что и в $\S 1$ (см. предложение 1.1). Заметим, что вероятностная мера, инвариантная относительно исходной группы $G$, существует тогда и только тогда, когда существует борелевская мера, конечная на компактах и инвариантная относительно групшы $\widehat{G}$. В таком случае утверждение теоремы будет следовать из теоремы 8.1, примененной к группе $\widehat{G}$.

Tеорема 8.3 [10]. Пусть $G \subseteq$ Нотео $_{+}(\mathbb{R})$. Тогда следующие утверждения эквивалентны:

(1) существует борелевская мера $\mu$, конечная на компактах и проективно-инвариантная относительно группь $G$;

(2) группа $G$ полусопряжсена некоторой группе ${ }_{*} G \subseteq \mathrm{Homeo}_{+}(\mathbb{R})$ аффинных преобразований прямой, сохраняющих ориентачию.

ЗАмЕчАниЕ 8.1. В теоремах 8.1, 8.3 мера $\mu$ и соответствующее отображение $\eta$ из определения полусопряженности связаны соотношением

$$
\eta(t)=\widetilde{\mu}([\bar{t}, t)), \quad \widetilde{\mu}([\bar{t}, t))= \begin{cases}+\mu([\bar{t}, t)), & \text { если } t \geqslant \bar{t} \\ -\mu((t, \bar{t}]), & \text { если } t<\bar{t},\end{cases}
$$

где $\bar{t} \in \mathbb{R}$ - некоторая заданная точка. Из непрерьвности отображения $\eta$ следует непрерьвность меры $\mu$ и наоборот.

Теперь мы можем сформулировать аналоги теорем 8.1-8.3 для групп $G \subseteq$ $\operatorname{Homeo}(X)$.

TeOpema 8.4. Пусть $G \subseteq \operatorname{Homeo(}(\mathbb{R})$. Тогда следующие утверждения эквивалентны:

(1) существует борелевская мера $\mu$, конечная на компактах и инвариантная относительно группь $G$;

(2) группа $G$ полусопряжена некоторой группе ${ }_{*} G \subseteq \operatorname{Homeo(}(\mathbb{R})$ изометрий.

ДоКАЗАТЕльство. Пусть существует мера $\mu$, инвариантная относительно группы $G$. Если $G=G_{+}$, то доказательство следует из теоремы 5.1. Пусть $G \neq G_{+}$. Тогда по теореме 8.1 подгруппа $G_{+}$будет полусопряжена некоторой подгрупше ${ }_{*} G_{+}$ групшы сдвигов. Отображение $\eta$ из определения полусопряженности задается с помощью формул из замечания 8.1. Мы знаем, что $G=G_{+} \cup f G_{+}$, где $f$ - некоторый элемент из $G \backslash G_{+}$. Остается показать, что с помошью отображения $\eta$ гомеоморфизм $f$ будет полусопряжен некоторой изометрии. Действительно, для любого $t \in \mathbb{R}$

$$
\eta(f(t))=\widetilde{\mu}([\bar{t}, f(t)))=\widetilde{\mu}([\bar{t}, f(\bar{t})))+\widetilde{\mu}([f(\bar{t}), f(t))) .
$$

Так как гомеоморфизм $f$ меняет ориентацию, а мера $\mu$ инвариантна относительно $f$, то

$$
\widetilde{\mu}([f(\bar{t}), f(t)))=-\widetilde{\mu}([\bar{t}, t))=-\eta(t) .
$$


Окончательно получим, что для любого $t \in \mathbb{R}$

$$
\eta(f(t))=\widetilde{\mu}([\bar{t}, f(\bar{t})))-\eta(t)
$$

т.е. отображение $f$ полусопряжено изометрии.

Проведем доказательство в обратную сторону. Пусть $G$ полусопряжена некоторой групе ${ }_{*} G \subseteq \operatorname{Homeo}(\mathbb{R})$ изометрий. Тогда $G_{+}$будет полусопряжена некоторой групе сдвигов и по теореме 8.1 сушествует мера, инвариантная относительно $G_{+} \cdot$ Тогда по теореме 5.10 сушествует мера, инвариантная относительно исходной групшы $G$.

ТеОрема 8.5. Пусть $G \subseteq \operatorname{Homeo}\left(S^{1}\right)$. Тогда следующие утверждения әквивалентны:

(1) существует вероятностная мера, инвариантная относительно груп$n b l \mathrm{G}$;

(2) әруппа $G$ полусопряжсена некоторой группе ${ }_{*} G \subseteq \operatorname{Homeo}\left(S^{1}\right)$ изометрий.

ДокАЗАТЕльство. Пусть $\widehat{G} \subseteq \operatorname{Homeo}\left(S^{1}\right)$ и отображения $\bar{g}, \lambda, \pi_{\widehat{G}}$ те же, что в $\S 1$ (см. предложение 1.1). Заметим, что вероятностная мера, инвариантная относительно исходной группы $G$, существует тогда и только тогда, когда сушествует борелевская мера, конечная на компактах и инвариантная относительно групшы $\widehat{G}$. В таком случае утверждение теоремы будет следовать из теоремы 8.4, примененной к группе $\widehat{G}$.

В теоремах 8.4 и 8.5 важно знать, как устроено ядро гомоморфизма $\eta^{\#}$ из определения полусопряженности.

СЛЕДСТВИЕ 8.1. В условиях теорем 8.4 и 8.5 справедливо равенство $\operatorname{ker} \eta^{\#}=H_{G}$.

ДокАЗАТЕльство. Очевидно, что достаточно провести доказательство для груп $G \subseteq$ Homeo+ $_{+}(\mathbb{R})$. По лемме $5.2 H_{G}=G^{s}$. Так как носитель инвариантной меры $\mu$ принадлежит непустому множеству $\operatorname{Fix} G^{s}$, то в силу замечания 8.1 справедливо равенство $\operatorname{ker} \eta^{\#}=G^{s}$.

Tеорема 8.6. Пусть $G \subseteq \operatorname{Homeo}(\mathbb{R})$. Тогда следующие утвержсдения әквивалентнь:

(1) существует борелевская мера $\mu$, конечная на компактах и проективно-инвариантная относительно группь $G$;

(2) группа $G$ полусопряжена некоторой группе ${ }_{*} G \subseteq \operatorname{Homeo}(\mathbb{R})$ аффинных преобразований прямой.

ДокАЗАТЕльство. Пусть существует мера $\mu$, проективно-инвариантная относительно группы $G$. Если $G=G_{+}$, то доказательство следует из теоремы 8.3. Пусть $G \neq G_{+}$. Тогда по теореме 8.1 подгрупп $G_{+}$будет полусопряжена некоторой подгрупе ${ }_{*} G_{+}$группы аффинных преобразований. Отображение $\eta$ из определения полусопряженности задается с помощью формул из замечания 8.1. Мы знаем, что $G=$ $G_{+} \cup f G_{+}$, где $f$ - некоторый элемент из $G \backslash G_{+}$. Остается показать, что с помошю отображения $\eta$ гомеоморфизм $f$ будет полусопряжен некоторому афффинному преобразованию. Действительно, для любого $t \in \mathbb{R}$

$$
\eta(f(t))=\widetilde{\mu}([\bar{t}, f(t)))=\widetilde{\mu}([\bar{t}, f(\bar{t})))+\widetilde{\mu}([f(\bar{t}), f(t))) .
$$


Так как гомеоморфизм $f$ меняет ориентацию, а мера $\mu$ - проективно-инвариантная относительно $f$, то

$$
\widetilde{\mu}([f(\bar{t}), f(t)))=-c(f) \widetilde{\mu}([\bar{t}, t))=-c(f) \eta(t),
$$

где $c(f)$ - некоторая положительная константа. Окончательно получим, что для любого $t \in \mathbb{R}$

$$
\eta(f(t))=\widetilde{\mu}([\bar{t}, f(\bar{t})))-c(f) \eta(t),
$$

т.е. отображение $f$ полусопряжено афффинному преобразованию.

Проведем доказательство в обратную сторону. Пусть $G$ полусопряжена некоторой групе ${ }_{*} G \subseteq \operatorname{Homeo}(\mathbb{R})$ афффинных преобразований. Тогда $G_{+}$будет полусопряжена некоторой группе аффинных преобразований, сохраняюших ориентацию, и по теореме 8.3 существует мера, проективно-инвариантная относительно $G_{+}$· Тогда по теореме 6.6 сушествует мера, проективно-инвариантная относительно исходной группы $G$.

В теореме 8.6 также важно знать, как устроено ядро гомоморфизма $\eta^{\#}$ из определения полусопряженности.

СлЕДСТВИЕ 8.1. Пусть для группы $G \subseteq \operatorname{Homeo}(\mathbb{R})$ не существует инвариантной меры. Тогда в условиях теоремы 8.6 справедливо равенство $\operatorname{ker} \eta^{\#}=H_{G}$.

ДокАЗАТЕЛЬСтво. Очевидно, что достаточно провести доказательство для групп $G \subseteq$ Homeo+ $_{+}(\mathbb{R})$. Так как для группы $G$ не существует инвариантной меры, то минимальное множество такой групшы недискретное (предложение 6.3). По теореме 6.3 носитель проективно-инвариантной меры такой группы совпадает с минимальньм множеством. Тогда в силу замечания 8.1 справедливо условие $\operatorname{ker} \eta^{\#}=H_{G}$.

В работе В.В. Солодова [41] для одного класса групп $G \subseteq \mathrm{Homeo+}_{+}(\mathbb{R})$ сформулирован результат об их полусопряженности некоторым группам афффинных преобразований прямой. Приведем его доказательство, основанное на теоремах 6.4 и 8.3.

ТеОрема 8.7 [41]. Пусть $G \subseteq$ Homeо+ $_{+}(\mathbb{R})$ - некоммутативная группа и для любого әлемента $g \in G \backslash$ м множество $\operatorname{Fix}\langle g\rangle$ состоит из не более чем одной точки. Тогда группа $G$ полусопряжена некоторой группе ${ }_{*} G \subseteq \operatorname{Homeo(}(\mathbb{R})$ аффинных преобразований прямой и при этом гомоморфизм $\eta^{\#}$ является изоморфизмом.

ДокАЗАТЕльство. Покажем, что для группы $G$ не сушествует инвариантной меры. Доказательство проведем от противного. Пусть существует инвариантная мера. Тогда по теореме 5.1 вьполняется условие Fix $G^{s} \neq \varnothing$. Так как для любого $g \in G \backslash e$ множество $\operatorname{Fix}\langle g\rangle$ состоит из не более чем одной точки, то либо $G^{s}=\langle e\rangle$, либо $G=G^{s}$. Если $G^{s}=\langle e\rangle$, то по теореме 2.1 группа $G$ коммутативная, чего не может быть. Если $G=G^{s}$, то, не ограничивая обшности, можем считать, что множество Fix $G^{s}$ совпадает с точкой 0 . Очевидно, что действие групшы $G$ на $\mathbb{R}$ распадается на действия на полупрямых $\left.\mathbb{R}_{-}=\right]-\infty, 0\left[, \mathbb{R}_{+}=\right] 0,+\infty[$. Рассмотрим ее действие на полупрямой $\left.\mathbb{R}_{+}=\right] 0,+\infty\left[\right.$. Применив преобразование $\theta: \mathbb{R} \rightarrow \mathbb{R}_{+}, \theta(t)=e^{t}$, получим действие индуцированной групшы на прямой. Элементы такой групшы, отличные от единищы, действуют свободно. Тогда по теореме 5.1 такая группа коммутативная. Точно так 
же мы поступим и с действием исходной группы наполупрямой $\left.\mathbb{R}_{-}=\right]-\infty, 0[$. В итоге получим, что исходная группа $G$ коммутативная, чего не может быть.

Заметим, что для группы $G$ справедливо условие $G_{\infty}^{s}=\langle e\rangle$. Поэтому для такой групы условие 1) теоремы 6.4 вьполняется.

Если элемент $g \in G \backslash e$ имеет неподвижную точку, то обозначим ее через $t_{g}$. Так как группа $G$ некоммутативная, а любой элемент имеет не более одной неподвижной точки, то график преобразования $g$ с неподвижной точкой $t_{g}$ справа от $t_{g}$ и слева от $t_{g}$ будет находиться по разные стороны от диагонали. Следовательно, для группы $G$ выполняется условие 2) теоремы 6.4.

Условие 3) теоремы 6.4 также выполняется, ибо любой элемент $g \in G \backslash e$ имеет не более одной неподвижной точки. Поэтому по теореме 6.4 для групшы $G$ сушествует проективно-инвариантная мера $\mu$, а по теореме 8.3 групша $G$ полусопряжена некоторой групте $* G$ аффинных преобразований прямой. По следствию $8.2 \mathrm{ker} \eta^{\#}=G_{\infty}^{s}$. Так как $G_{\infty}^{s}=\langle e\rangle$, то гомоморфизм $\eta^{\#}$ является изоморфизмом.

Важньп классом груп $G \subseteq$ Hотео+ $_{+}(\mathbb{R})$ являются группы, топологически сопряженные группам $* G \subseteq \widetilde{\text { Нотео }_{+}}\left(S^{1}\right)$, элементы которых являются накрытиями окружности. Приведем критерий такой топологической сопряженности.

ПредЛОЖЕНИЕ 8.1 [10]. Пусть $G \subseteq$ Нотео $_{+}(\mathbb{R})$. Группа $G$ топологически сопряжена группе ${ }_{*} G \subseteq$ Нотео $_{+}\left(S^{1}\right)$ тогда и только тогда, когда существует гомеоморфизм $\widehat{g} \in \mathrm{Homeo}_{+}(\mathbb{R})$ такой, что:

1) гомеоморфизм $\widehat{g}$ свободно действует на прямой;

2) $\widehat{g}$ перестановочен со всеми әлементами группь $G$.

Вьше были даны критерии полусопряженности групшы $G \subseteq$ Homeo $(\mathbb{R})$ групе сдвигов и группе афффинных преобразований, что было эквивалентно существованию инвариантной и проективно-инвариантной меры соответственно. Для формулировки критерия полусопряженности группы $G \subseteq \mathrm{Homeo}_{+}(\mathbb{R})$ с $\omega$-проективно-инвариантной мерой некоторой канонической группе ${ }_{*} G \subseteq \mathrm{Homeo}_{+}(\mathbb{R})$ необходимо дать эквивалентные переформулировки теорем 8.4-8.6.

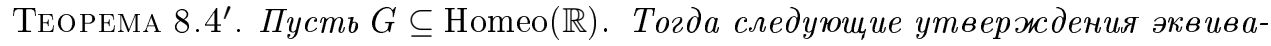
лентны:

(1) существует борелевская мера $\mu$, конечная на компактах и инвариантная относительно группь $G$;

(2) группа $G$ полусопряжена некоторой группе ${ }_{*} G \subseteq \operatorname{Homeo(}(\mathbb{R})$, относительно которой мера Лебега $d t$ является инвариантной.

Теорема 8.5'. Пусть $G \subseteq \operatorname{Homeo}\left(S^{1}\right)$. Тогда следующие утверждения әквивалентны:

(1) существует вероятностная мера, инвариантная относительно груп$n$ вь $G$;

(2) группа $G$ полусопряжена некоторой группе ${ }_{*} G \subseteq \operatorname{Homeo}\left(S^{1}\right)$, относительно которой мера Лебега $d t$ является инвариантной. 
TeOPEмa 8.6'. Пусть $G \subseteq$ Нотео $(\mathbb{R})$. Тогда следующие утверждения эквивалентны:

(1) существует борелевская мера $\mu$, конечная на компактах и проективно-инвариантная относительно группь $G$;

(2) группа $G$ полусопряжена некоторой группе ${ }_{*} G \subseteq \operatorname{Homeо}(\mathbb{R})$, относительно которой мера Лебега $d t$ является проективно-инвариантной.

Теперь мы можем сформулировать гипотезу о полусопряженности для групп с $\omega$-проективно-инвариантной мерой.

ГиПотезА 8.1. Пусть $G \subseteq$ Нотео+ $_{+}(X)$. Тогда следующие утверждения эквивалентны:

(1) существует борелевская мера $\mu$, конечная на компактах и $\omega$-проективно-инвариантная относительно группь $G$;

(2) группа $G$ полусопряжсена некоторой группе ${ }_{*} G \subseteq \mathrm{Homeo}_{+}(X)$, относительно которой мера Лебега $d t$ является $\omega$-проективно-инвариантной.

\section{§. О комбинаторных свойствах групп гомеоморфизмов прямой и окружности}

Мы уже установили, что для групп гомеоморфизмов прямой с непустьм минимальным множеством существует метрический инвариант в виде $\omega$-проективно-инвариантной меры, где $\omega$ - некоторое кардинальное число (теорема 7.4). Естественно поставить вопрос: какие дополнительные условия необходимы (или, что то же, какие сушествуют "препятствия"), чтобы $\omega$-проективно-инвариантная мера оказалась инвариантной либо проективно-инвариантной? Оказьвается, эти "препятствия" имеют комбинаторньй характер и связаны с комбинаторными характеристиками фактор-групшы исходной групшы по максимальной нормальной подгруппе с инвариантной мерой, исследованной в $\S 7$. Центральньй результат по этой проблеме приводится в теоремах 9.3 и 9.8. У Уочнение теоремы 9.3 для группы гомеоморфизмов окружности (теорема 9.7) является неулучшаемым усилением гипотезы Гиза о сушествовании инвариантной меры, доказанной Г. Маргулисом (теорема 9.2). Используя теорему о фактор-групе, полученные результаты о существовании инвариантной, либо проективно-инвариантной меры удается переформулировать в виде аналогов альтернативы Титса, используюшие только лишь комбинаторные характеристики исходной групш (теоремы 9.4' $9.7^{\prime}, 9.8^{\prime}$ ).

Далее будет сформулирована замечательная теорема Планте о критерии существования инвариантной меры для конечно-порожденных групा $G \subseteq \mathrm{HomeO}_{+}(\mathbb{R})$ (теорема 9.1). K сожалению, теорема Планте не допускает распространения на случай не конечно-порожденных групп. Для произвольных груп $G \subseteq \mathrm{Homeo+}_{+}(\mathbb{R})$ описано комбинаторное "препятствие" для существования проективно-инвариантной меры (теорема 9.3), а также его модификации (теорема 9.4). Отмеченные результаты получены и для обших групा $G \subseteq \operatorname{Homeo}(\mathbb{R})$ (теорема 9.5). В случае групा $G \subseteq \widetilde{\operatorname{Homeo}}\left(S^{1}\right)$ и $G \subseteq \operatorname{Homeo}\left(S^{1}\right)$ такое "препятствие" становится комбинаторным "препятствием" для сушествования уже инвариантной меры (теоремы 9.6, 9.7). Для групा $G \subseteq \operatorname{Homeo(} \mathbb{R})$ 
сформулирован другой комбинаторньй критерий сушествования инвариантной меры (теорема 9.8). Все отмеченные критерии существования метрических инвариантов для групп $G \subseteq \operatorname{Homeo}(X)$ допускают переформулировку в терминах комбинаторных характеристик исходной групшы в виде аналога альтернативы Титса (теоремы $\left.9.4^{\prime}, 9.7^{\prime}, 9.8^{\prime}\right)$. Для этого будут сформулированы предварительные результаты для групп $G \subseteq \mathrm{Homeo}_{+}(X)$ (леммы 9.3-9.5). Приводятся конструкции свободной подгрупшы с двумя образуюшими из $\widetilde{\mathrm{Homeo}}\left(S^{1}\right)$ (лемма 9.1$)$ и свободной подполугруппы с двумя образуюшими из $\mathrm{Homeo+}_{+}(\mathbb{R})$ (лемма 9.2).

9.1. Проблема существования инвариантных и проективно-инвариантных мер и связанные с ними комбинаторные “препятствия". Вначале результат такого типа сформулируем для конечно-порожденных груп. Для таких групп известно, что минимальное множество всегда непусто (теорема 3.4 ).

ОпредЕлЕниЕ 9.1. Конечно-порожденная группа $G \subseteq \mathrm{Homeo}_{+}(\mathbb{R})$ имеет неэкспоненциальный рост, если

$$
\liminf _{n \rightarrow \infty} \frac{1}{n} \log \left|G^{n}\right|=0
$$

где $G^{n}$ - слова длины не более чем $n$, а $\left|G^{n}\right|-$ их мошность. Орбита $G(t)$ точки $t \in \mathbb{R}$ имеет неэкспоненциальньй рост, если

$$
\liminf _{n \rightarrow \infty} \frac{1}{n} \log \left|G^{n}(t)\right|=0 .
$$

Сформулируем замечательную теорему Планте, в которой для конечно-порожденной группы приводится комбинаторный критерий существования инвариантной меры.

Tеорема 9.1 [32]. Пусть $G \subseteq$ Homeo+ $_{+}(\mathbb{R})$ - конечно-порожденная группа. Для существования борелевской меры, конечной на компактах и инвариантной относительно группь $G$, необходимо и достаточно, чтобь существовала точка $t \in \mathbb{R}$, орбита которой имеет неэкспоненциальный рост.

$\mathrm{K}$ сожалению, теорема Планте не допускает обобщения на произвольные группы. В работе [28] Г. Маргулисом была доказана гипотеза Гиза.

Теорема 9.2 [28]. Для группь $G \subseteq \operatorname{Homeо}\left(S^{1}\right)$ или существует свободная подгруппа с двумя образующими, или существует вероятностная инвариантная мера на $S^{1}$.

Результат, эквивалентный альтернативе из теоремы 9.2 , получен в работе В. В. Солодова [40]. В указанной работе условие существования инвариантной меры заменено на эквивалентное (в силу теоремы 5.14) условие: псевдохарактер $\tau_{G}$ является характером.

Очевидно, что альтернатива из теоремы 9.2 является усилением теоремы Дэйя для случая окружности: для аменабельной группы $G \subseteq \operatorname{Homeo}\left(S^{1}\right)$ существует вероятностная инвариантная мера.

Неулучшаемое усиление альтернативы из теоремы 9.2 может быть получено как следствие нижеследующей теоремы 9.3 и сформулировано в виде теоремы 9.7.

В конще раздела 7.1 ставился вопрос о критерии существования проективно-инвариантной меры в терминах фактор-групшы $G / H_{G}$. Оказьвается, что для групп $G$ с нетривиальной фактор-группой $M_{0, G} / H_{G}$ такой критерий можно сформулировать. 
Tеорема 9.3 [11]. Пусть $G \subseteq$ Homeo+ $_{+}(\mathbb{R})$, для групnь $G$ существует непустое минимальное множсество, а фактор-группа $M_{0, G} / H_{G}$ нетривиальная. Тогда для существования проективно-инвариантной борелевской меры, конечной на компактах, необходимо и достаточно, чтобы фактор-группа $G / H_{G}$ не содержала свободных подгрупп с двумя образующими.

Напомним, что в теореме 9.3 условие непустоты минимального множества и нетривиальности фактор-группы $M_{0, G} / H_{G}$ в силу леммы 5.1 и предложения 7.1 допускает еще две эквивалентные переформулировки:

1) для групшы $G$ сушествует нормальная подгруппа $\Gamma$ с инвариантной мерой и свободно действуюшим элементом;

2) для групшы $G$ существует нормальная подгрупа $\Gamma$ с нетривиальной факторгруппой $\Gamma /\left\langle\Gamma^{s}\right\rangle$.

Дадим эквивалентную переформулировку теоремы 9.3 .

TеOрема 9.4. Пусть группа $G \subseteq \mathrm{Homeo}_{+}(\mathbb{R})$ содержит нормальную подгруппу Г с инвариантной мерой и свободно действующим әлементом. Для существования борелевской мерь, конечной на компактах и проективно-инвариантной относительно группь $G$, необходимо и достаточно, чтобы фактор-группа $G / H_{G}$ не содержала свободной подгруппь с двумя образующими.

В работе Ж.-Ф. Планте [33] приводится доказательство достаточного условия теоремы 9.4 при более сильном предположении, что фактор-группа $G / \Gamma$ является аменабельной (фактор-группы $G / \Gamma$ и $G / H_{G}$ аменабельны одновременно). В той же работе [33] доказано сушествование проективно-инвариантной меры для специальных разрешимых групп.

В [16] утверждение теоремы 9.3 было получено для произвольной групшы $G \subseteq$ $\operatorname{Homeo}(\mathbb{R})$, которую мы и приводим.

Tеорема $9.5[16]$. Пусть группа $G \subseteq \operatorname{Homeo}(\mathbb{R})$ содержит нормальную подгруппу $\Gamma$ с инвариантной мерой и свободно действующим әлементом. Для существования борелевской меры, конечной на компактах и проективно-инвариантной относительно группь $G$, необходимо и достаточно, чтобь фактор-группа $G / H_{G}$ не содержсла свободной подгруппьг с двумя образующими.

Для групш $G \subseteq \widetilde{\operatorname{Homeo}}\left(S^{1}\right)$ теорема 9.5 может быть уточнена.

Теорема 9.6. Пусть $G \subseteq \widetilde{\operatorname{Homeo}}\left(S^{1}\right)$. Тогда для существования борелевской меры, конечной на компактах и инвариантной относительно группы $G$, необходимо и достаточно, чтобь фактор-группа $G / H_{G}$ не содержсала свободной подгруппье с двумя образующими.

ДокАЗАТЕльство. Заметим, что для групшы $G$ инвариантная мера существует тогда и только тогда, когда сушествует инвариантная мера для подгруппы $G_{+}$(теорема 5.10). С другой стороны, фактор-группы $G / H_{G}, G_{+} / H_{G}$ не содержат свободных подгрупп с двумя образуюшими одновременно. Поэтому достаточно доказательство провести для групш $G \subseteq \widehat{\mathrm{Homeo}_{+}}\left(S^{1}\right)$.

По предложению 6.5 для групш $G$ проективно-инвариантная мера сушествует тогда и только тогда, когда сушествует инвариантная мера. Образуем групу П $=\langle G, \bar{g}\rangle$, 
где $\bar{g}(t)=t+1$. Для произвольной группы $Q \subseteq \widetilde{\text { Нотео }_{+}}\left(S^{1}\right)$ минимальное множество непусто (теорема 3.6 ), а инвариантная мера существует тогда и только тогда, когда сушествует инвариантная мера для группы $\langle Q, \bar{g}\rangle$ (предложение 5.5). Отсюда следует, что для группы П 0-максимальная подгруппа $M_{0, \Pi}$ сушествует и содержит элемент $\bar{g}$. Поэтому $M_{0, \Pi} \neq H_{\Pi}$ и для группы П вьполняются условия теоремы 9.3 . Для завершения доказательства остается заметить, что фактор-групшы $G / H_{G}$ и $\Pi / H_{П}$ одновременно либо содержат свободную подгрупшу с двумя образуюшими, либо ее не содержат.

Теперь мы можем сфформулировать и доказать аналог теоремы 9.5 для групा, действуюших на окружности.

Теорема 9.7 [16]. Пусть $G \subseteq \operatorname{Homeo}\left(S^{1}\right)$. Для существования вероятностной мерь, инвариантной относительно группы $G$, необходимо и достаточно, чтобь фактор-группа $G / H_{G}$ не содержала свободной подгруппь с двумя образующими.

ДокАЗАТЕльство. Заметим, что для группы $G$ инвариантная вероятностная мера сушествует тогда и только тогда, когда существует инвариантная вероятностная мера для подгрупшы $G_{+}$(теорема 5.10). С другой стороны, фактор-групшы $G / H_{G}$, $G_{+} / H_{G}$ не содержат свободных подгрупп с двумя образуюшими одновременно. Поэтому достаточно провести доказательство для групп $G \subseteq \mathrm{Homeo}_{+}\left(S^{1}\right)$.

Для элемента $g \in G$ через $l_{g}$ обозначим гомеоморфизм прямой, являюшийся накрытием гомеоморфизма окружности $g$ и нормированный условием $0 \leqslant l_{g}(0)<1$. Образуем группу $\widehat{G}=\left\langle\left\{l_{g}\right\}_{g \in G}, \bar{g}\right\rangle$, где $\bar{g}(t)=t+1$. Для группы $G$ инвариантная мера существует тогда и только тогда, когда она существует для группы $\widehat{G}$. Пусть $\Gamma=$ $\left\langle H_{\widehat{G}}, \bar{g}\right\rangle$. Ядро естественного эпиморфизма $\pi: \widehat{G} \rightarrow G$ равно $\operatorname{ker} \pi=\langle\bar{g}\rangle$ и $\pi(\Gamma)=H_{G}$. Следовательно, фактор-группы $\widehat{G} / \Gamma$ и $G / H_{G}$ изоморфны. С другой стороны, фактор-групшы $\widehat{G} / \Gamma$ и $\widehat{G} / H_{\widehat{G}}$ одновременно либо содержат свободную подгруппу с двумя образуюшими, либо ее не содержат. В таком случае для группы $\widehat{G}$ выполняются все условия теоремы 9.4, откуда и следует доказательство.

ЗАмечание 9.1. Для групшы $G \subseteq$ Homeo+ $_{+}(X)$ фактор-группа $G / H_{G}$ может быть реализована как группа действий на $X$. Для любого класса смежности $g H_{G}$ соответствующее ей действие на минимальном множестве совпадает с действием элемента $g$, а вне минимального множества действует растяжениями на максимальных открытых интервалах. При этом инвариантная мера для группы $G$ является инвариантной мерой для фактор-группы $G / H_{G}$ и наоборот.

Альтернатива, сформулированная в теореме 9.7, является неулучшаемым усилением альтернативы, доказанной Маргулисом (теорема 9.2). Заметим, что в случае $H_{G}=\langle e\rangle$ теоремы 9.2 и 9.7 эквивалентны, т.е. слабая альтернатива эквивалентна сильной. Для справедливости равенства $H_{G}=\langle e\rangle$ достаточно, чтобы минимальное множество группы $G$ совпадало со всей прямой. Этот факт отмечен в работе Э. Гиза $[19]$.

Теперь мы попытаемся для груп $G \subseteq \operatorname{Homeo}(\mathbb{R})$ сфформулировать критерий существования инвариантной меры. Очевидно, что, в отличие от критерия существования проективно-инвариантной меры (теорема 9.4), условия на фактор-группу $G / H_{G}$ должны быть более жесткими. 
Теорема 9.8 [16]. Пусть $G \subseteq \operatorname{Homeo}(\mathbb{R})$. Для существования борелевской ме$p b$, конечной на компактах и инвариантной относительно группь $G$, необходимо и достаточно, чтобы выполнялись условия:

(1) для группь $G$ существует непустое минимальное множсество;

(2) фактор-группа $G / H_{G}$ не содержит свободной подполугруппь с двумя образующими.

Напомним, что для конечно-порожденных груп минимальное множество непусто. В работе В.В. Солодова [39] для специальных конечно-порожденных групп $G \subseteq \mathrm{Homeo+}_{+}(\mathbb{R})$ получен результат, эквивалентньй более сильному достаточному условию: если группа $G$ не содержит свободной подполугруппь с двумя образующими, то множество Fix $G^{s}$ непусто (в силу теоремы 5.1 последнее условие эквивалентно условию сушествования инвариантной борелевской меры, конечной на компактах).

Если для любого элемента групшы $G \subseteq \operatorname{Homeo}(\mathbb{R})$ множество неподвижных точек дискретное, то по теореме 3.3 минимальное множество такой групшы непусто. Если группа абелева, то для нее справедливо условие (2) теоремы 9.8, так как она не может содержать неабелевой свободной подполугрупшы с двумя образующими. Следовательно, для таких груп существует инвариантная мера. Такая теорема доказана в работе Э. Планте [32]. В другой работе Э. Планте [33] для абелевых групп $G \subseteq \mathrm{Homeo+}_{+}(\mathbb{R})$ также сформулированы признаки сушествования инвариантной меры. Такими признаками являются наличие свободно действующего гомеоморфизма $g \in G$ либо условие принадлежности элементов группы $G$ пространству $\operatorname{Diff}^{2}(\mathbb{R})$. В силу теорем 3.3 и 3.5 для таких групп минимальное множество непусто. Так как группа абелева, то она не может содержать неабелевой свободной подполугрупшы с двумя образуюшими. Следовательно, для таких групп по теореме 9.8 будет сушествовать инвариантная мера.

И наконец, приведем две леммы Солодова. В первой строится свободная группа $G \subseteq \widetilde{\text { Нотео }}\left(S^{1}\right)$ с двумя образуюшими, а во второй строится свободная подполугруппа $G \subseteq \mathrm{Homeo+}_{+}(\mathbb{R})$ с двумя образуюшими.

Лемма 9.1 [40]. Eсли $g_{1}, g_{2} \in G \subseteq \widehat{\operatorname{Homeo}}\left(S^{1}\right), \tau\left(g_{1}\right)=0, \tau\left(g_{2}\right)=0$, но $\tau\left(g_{1} g_{2}\right) \neq 0$, то между некоторыми степенями $g_{1}$ и $g_{2}$ нет нетривиальных соотношений.

Лемма 9.2 [39]. Если $g_{1}, g_{2} \in \operatorname{Homeo}_{+}(\mathbb{R}), a, b \in \mathbb{R}, a>b, g_{1}(a)=a, g_{2}(b)=b$, преобразование $g_{1}$ не имеет неподвижных точек в интервале $(c, a), b \in(c, a), a$ преобразование $g_{2}$ не имеет неподвижных точек в интервале $(b, d), a \in(b, d)$, то группа, порожденная $g_{1}$ и $g_{2}$, содержит свободную подполугруппу с двумя образующими.

В теореме 9.4 предполагалось существование специальной нормальной подгруппы Г. Можно ли отказаться от такого априорного предположения за счет усиление иных условий? Ответ на такой вопрос содержится в следующей гипотезе.

ГиПотеЗА 9.1. Пусть $G \subseteq$ Нотео+ $_{+}(\mathbb{R})$. Для существования борелевской меpbl, конечной на компактах и проективно-инвариантной относительно группьи $G$, необходимо и достаточно, чтобь фактор-группа $G / H_{G}$ была аменабельной. 
9.2. Об аналогах альтернативы Титса для групп гомеоморфизмов прямой и окружности. Приводимые критерии существования проективно-инвариантной и инвариантной мер в виде альтернативы используют как метрические, так и комбинаторные характеристики групшы. Хотелось бы сформулировать альтернативы, используюшие только лишь комбинаторные характеристики групшы. Таким примером служит знаменитая альтернатива Титса [51]: конечно-порожденная линейная группа либо содержит свободную подгруппу с двумя образующими, либо является почти разрешимой.

Далее будут сформулированы аналоги альтернативы Титса для групп гомеоморфизмов прямой и окружности, которые являются переформулировками соответствующих альтернатив из раздела 9.1.

Сформулируем предварительную лемму.

Лемма 9.3 [16]. Пусть группа $G \subseteq$ Hотео+ $(\mathbb{R})$ содержит нормальную подгруппу $\Gamma$ с инвариантной мерой и свободно действующим әлементом. Тогда фактор-группа $G / H_{G}$ либо содержит свободную подгруппу с двумя образующими, либо является разрешимой ступени не более двух.

Теперь мы можем переформулировать теорему 9.4 о существовании проективно-инвариантной мерой в виде аналога альтернативы Титса.

Teорема 9.4' [16]. Пусть группа $G \subseteq$ Hотео $(\mathbb{R})$ содержит нормальную подгруппу $\Gamma \subseteq \mathrm{Homeo}_{+}(\mathbb{R})$ с инвариантной мерой и свободно действующ,им әлементом. Тогда фактор-группа $G / H_{G}$ либо содержит свободную подгруппу с двумя образуюшими, либо содержит разрешимую подгруппу ступени и индекса не более двух.

Критерий сушествования инвариантной меры для групп гомеоморфизмов окружности, приводимый в теореме 9.7, также допускает переформулировку в виде аналога альтернативы Титса. Сформулируем предварительную лемму.

Лемма 9.4 [16]. Для группь $G \subseteq$ Homeo+ $\left(S^{1}\right)$ фактор-группа $G / H_{G}$ либо содержит свободную подгруппу с двумя образующими, либо является коммутативной.

Теперь мы можем сформулировать аналог альтернативы Титса.

Teopema 9. $7^{\prime}[16]$. Для группь $G \subseteq \operatorname{Homeo}\left(S^{1}\right)$ фактор-груnпа $G / H_{G}$ либо содержит свободную подгруппу с двумя образующими, либо содержит коммутативную подгруппу индекса не более двух.

Критерий существования инвариантной меры для груп гомеоморфизмов прямой, приводимьй в теореме 9.8, также допускает переформулировку в виде аналога альтернативы Титса. Сформулируем предварительную лемму.

Лемма $9.5[16]$. Для группы $G \subseteq$ Нотео $_{+}(\mathbb{R})$ с непустыц минимальным множеством фактор-группа $G / H_{G}$ либо содержит свободную подполугруппу с двумя образующими, либо является коммутативной.

Теперь мы можем сформулировать аналог альтернативы Титса. 
Tеорема 9.8' [16]. Для группы $G \subseteq \operatorname{Homeо}(\mathbb{R})$ с непустыцм минимальныцм множеством фактор-группа $G / H_{G}$ либо содержит свободную подполугруппу с двумя образующими, либо содержит коммутативную подгруппу индекса не более двyx.

\section{СПИСОК ЛИТЕРАТУРЫ}

[1] Л.В.Альфорс. Лекции по квазиконформным отображениям. М.: Мир, 1969.

[2] Д. В. Аносов. О вкладе Н. Н. Боголюбова в теорию динамических систем // УМН. 1994. T. 49. № 5. C. 5-20.

[3] A. Antonevich, A. Lebedev. Functional-differential Equations. I. $C^{*}$-theory. Harlow: Longman, 1994.

[4] В.И. Арнольд. Малые знаменатели. І. Об отображениях окружности на себя // Изв. АН СССР. Сер. матем. 1961. Т. 25. №1. С. 21-86.

[5] В.И. Арнольд. Дополнительные главы теории обыкновенных дифференциальных уравнений. М.: Наука, 1978.

[6] Л.А.Бекларян. О приводимости дифференциальных уравнений с отклоняющимся аргументом к уравнению с постоянными соизмеримыми отклонениями // Матем. заметки. 1988. T. 44. № 5. C. 561-565.

[7] Л.А. Бекларян. Об одном методе регуляризации краевых задач для дифференциальных уравнений с отклоняющимся аргументом // Докл. АН СССР. 1991. Т. 317. № 5 . C. $1033-1038$.

[8] Л.А. Бекларян. Задача оптимального управления для систем с отклоняющимся аргументом и ее связь с конечно-порожденной группой гомеоморфизмов $\mathbb{R}$, порожденной функциями отклонения // Докл. АН СССР. 1991. Т. 317. №6. С. 1289-1294.

[9] Л.А. Бекларян. К вопросу о классификации групп гомеоморфизмов $\mathbb{R}$, сохраняющих ориентацию. І. Инвариантные меры. // Матем. сб. 1996. Т. 187. № 3. С. 23-54.

[10] Л. А. Бекларян. K вопросу о классификации групп гомеоморфизмов $\mathbb{R}$, сохраняющих ориентацию. ІІ. Проективно-инвариантные меры // Матем. сб. 1996. Т. 187. № 4. С. 3-28.

[11] Л.А. Бекларян. K вопросу о классификации групп гомеоморфизмов $\mathbb{R}$, сохраняющих ориентацию. III. $\omega$-проективно-инвариантные меры // Матем. сб. 1999. Т. 190. №4. C. $43-62$.

[12] Л.А.Бекларян. Групповые особенности диффференциальных уравнений с отклоняющимся аргументом и связанные с ними метрические инварианты // Итоги науки и техники. Совр. матем. и ее прил. Тематические обзоры. 1999. Т. 67. С. 161-182.

[13] Л.А. Бекларян. Критерий существования проективно-инвариантной меры для групп гомеоморфизмов $\mathbb{R}$, сохраняющих ориентацию, связанный со структурой множества неподвижных точек // УМН. 1996. Т. 51. № 3. С. 179-180.

[14] Л.А. Бекларян. О критерии топологической сопряженности квазисимметрической группы группе афффинных преобразований $\mathbb{R}$ // Матем. сб. 2000. Т. 191. № 6. С. 31-42.

[15] L. A. Beklaryan. About canonical types of the differential equations with deviating argument // Funct. Differential Equations. 2001. V. 8. № 1-2. P. 25-33.

[16] Л. А. Бекларян. Об аналогах альтернативы Титса для групп гомеоморфизмов окружности и прямой // Матем. заметки. 2002. Т. 71. №3. С. 334-347.

[17] П. де ля Арп, Р. И. Григорчук, Т. Чекерини-Сильберстайн. Аменабельность и парадоксальные разбиения для псевдогрупп и дискретных метрических пространств // Труды МИАН. 1999. Т. 224. С. 68-111.

[18] É. Ghys. Groupes d'homéomorphismes du cercle et cohomologie bornée // Contemp. Math. 1987. V. 58. P. 81-106.

[19] É. Ghys. Groups acting on the circle // Enseign. Math. (2). 2001. V. 47. № 3-4. P. 329-407.

$[20] \Phi$. Гринлиф. Инвариантные средние на топологических группах и их приложения. М.: Мир, 1973. 
[21] R. I. Grigorchuk. Some results on bounded cohomology // London Math. Soc. Lecture Note Ser. 1995. V. 204. P. 111-163.

[22] Р.И. Григорчук, П.Ф. Курчанов. Некоторые вопросы теории групп, связанные с геометрией // Итоги науки и техники. Совр. пробл. матем. Фундам. напр. 1990. Т. 58. C. $191-256$.

[23] G. Hector, U. Hirsch. Introduction to the Geometry of Foliations. Part B. Foliations of Codimension One. Braunschweig: Vieweg, 1983.

[24] A. Hinkkanen. The structure of certain quasisymmetric groups // Mem. Amer. Math. Soc. 1990. V. 83. № 422 .

[25] H. Imanishi. Structure of codimension 1 foliations without holonomy on manifolds with abelian fundamental group // J. Math. Kyoto Univ. 1979. V. 19. № 3. P. 481-495.

[26] Ю. И. Карлович. $C$-алгебра операторов типа свертки с дискретными группами сдвигов и осциллирующими коэффициентами // Докл. АН СССР. 1988. Т. 302. № 3. С. 535-540.

[27] И.П. Корнфельд, Я.Г. Синай, С.В.Фомин. Эргодическая теория. М.: Наука, 1980.

[28] G. Margulis. Free subgroups of the homeomorphism group of the circle // C.R. Acad. Sci. Paris Sér. I Math. 2000. V. 331. №9. P. 669-674.

[29] S. Matsumoto. Numerical invariants for semiconjugacy of homeomorphisms of the circle // Proc. Amer. Math. Soc. 1986. V. 98. № 1. P. 163-168.

[30] S. Matsumoto. Some remarks on foliated $S^{1}$ bundles // Invent. Math. 1987. V. 90. № 2 . P. $343-358$.

[31] С. П. Новиков. Топология слоений // Труды ММО. 1965. Т. 14. С. 248-278.

[32] J.F. Plante. Foliations with measure preserving holonomy // Ann. of Math. (2). 1975. V. 102. № 2. P. 327-361.

[33] J. F. Plante. Solvable groups acting on the line // Transl. Amer. Math. Soc. 1983. V. 278. № 1. P. 401-414.

[34] А. Пуанкаре. Избранные труды. Т. I, II. М.: Наука, 1992.

[35] E. Salhi. Sur les ensembles minimaux locaux // C.R. Acad. Sci. Paris. Sér. I Math. 1982. V. 295. № 12. P. 691-694.

[36] E. Salhi. Sur un théorème de structure de feuilletages de codimension 1 // C.R. Acad. Sci. Paris. Sér. I Math. 1985. V. 300. № 18. P. 635-638.

[37] E. Salhi. Niveau des feuilles // C.R. Acad. Sci. Paris. Sér. I Math. 1985. V. 301. № 5. P. 219-222.

[38] Я. Г. Синай. Введение в эргодическую теорию. М.: Фазис, 1996.

[39] В.В.Солодов. Гомеоморфизмы прямой и слоения // Изв. АН СССР. Сер. матем. 1982. T. 46. № 5. С. 1047-1060.

[40] В. В. Солодов. Гомеоморфизмы окружности и слоения // Изв. АН СССР. Сер. матем. 1984. Т. 48. № 3. С. 599-613.

[41] В. В. Солодов. Топологические проблемы в теории динамических систем // УМН. 1991. T. 46. № 4. C. 93-114.

[42] А. И. Кокорин, В. М. Копытов. Линейно упорядоченные группы. М.: Мир, 1972.

[43] А. Г. Курош. Лекции по общей алгебре. М.: Наука, 1973.

[44] A. Banyaga. The Structure of Classical Diffeomorphism Groups. Dordrecht: Kluwer, 1997. (Math. Appl. V. 400.)

[45] M. G. Brin. The chameleon groups of Richard J. Thompson: automorphisms and dynamics // Inst. Hautes Études Sci. Publ. Math. 1996. V. 84. P. 5-33.

[46] M. G. Brin, M. Guzmán. Automorphisms of generalized Thompson groups // J. Algebra. 1998. V. 203. № 1. P. 285-348.

[47] J. W. Cannon, W. J. Floyd, W. R. Parry. Introductory notes on Richard Thompson's groups // Enseign. Math. (2). 1996. V. 42. № 3-4. P. 215-256.

[48] D. B. A. Epstein, K. Fujiwara. The second bounded cohomology of word-hyperbolic groups // Topology. 1997. V. 36. №6. P. 1275-1289. 
[49] N. Kovačevic. Möbius-like groups of homeomorphisms of the circle // Trans. Amer. Math. Soc. 1999. V. 351. № 12. P. 4791-4822.

[50] M. Stein. Groups of piecewise linear homeomorphisms // Trans. Amer. Math. Soc. 1992. V. 332. № 2. P. 477-514.

[51] J. Tits. Free subgroups in linear groups // J. Algebra. 1972. V. 20. P. 250-270.

[52] Л. Альфорс. Преобразования Мёбиуса в многомерном пространстве. М.: Мир, 1986.

[53] А. П. Робертсон, В. Дж. Робертсон. Топологические векторные пространства. М.: Мир, 1967.

Центральньй экономико-математический институт

Поступила в редакцию

E-mail: beklar@cemi.rssi.ru

08.04 .2004 